\title{
COSMOI OF INTERNAL CATEGORIES
}

\author{
BY
}

\author{
ROSS STREET
}

\begin{abstract}
An internal full subcategory of a cartesian closed category $\mathbb{Q}$ is shown to give rise to a structure on the 2-category $\operatorname{Cat}(\mathbb{Q})$ of categories in $\mathbb{Q}$ which introduces the notion of size into the analysis of categories in $\mathbb{Q}$ and allows proofs by transcendental arguments. The relationship to the currently popular study of locally internal categories is examined.

Internal full subcategories of locally presentable categories (in the sense of Gabriel-Ulmer) are studied in detail. An algorithm is developed for their construction and this is applied to the categories of double categories, triple categories, and so on.
\end{abstract}

Introduction. The theory of categories is essentially algebraic in the terminology of Freyd [12]. This means that it is pertinent to take models of the theory in any finitely complete category $\mathcal{Q}$. Of course this does not mean that all the usual properties of categories are available to us in $\mathcal{Q}$. The 2-category $\operatorname{Cat}(\mathbb{Q})$ of categories in $\mathcal{Q}$ is finitely complete (Street [29]) so pullbacks, categories of Eilenberg-Moore algebras for monads, comma categories, and so on, are available. Even some colimit constructions such as the categories of Kleisli algebras exist. Obviously the more like the category Set of sets $\mathbb{Q}$ becomes the more closely category theory internal to $\mathbb{Q}$ resembles ordinary category theory. If $\mathbb{Q}$ is finitely cocomplete, for example, we gain localization; or, if $\mathbb{Q}$ is cartesian closed we gain functor categories.

Yet there is an aspect of category theory which distinguishes it from other essentially algebraic theories; namely, the question of size. We take the position that size must be introduced by the endowment of extra data which is not universally determined by the category $\mathbb{Q}$ (as are limits, subobject classifiers, cartesian internal homs). In the present paper the extra data are taken to constitute an internal full subcategory of $\mathbb{Q}$. This means a category $S$ in $\mathbb{Q}$ together with a fully faithful representation of the internal arrows of $S$ as actual arrows in $\mathbb{Q}$. It then makes sense to ask whether or not a category $A$ in $Q$ has an $S$-valued homfunctor $A^{\text {op }} \times A \rightarrow S$; those $A$ which do are called admissible (or locally small) relative to the size structure on $\mathcal{Q}$ determined by our given internal full subcategory. It is shown in $\$ 10$ that, for a category $\mathbb{Q}$ sufficiently like Set (in fact, a Grothendieck topos), there must be a trade-off between the number of admissible categories in $\mathbb{Q}$

Received April 12, 1976 and, in revised form, December 15, 1978.

AMS (MOS) subject classifications (1970). Primary 18A15, 18C10, 18D05, 18D15, 18D30, 18D35, $18 \mathrm{~F} 10,55 \mathrm{~F} 35$.

Key words and phrases. Internal full subcategory, locally presentable category, locally small, fibred category, site, sketched structures, Gabriel theory, internally complete, cartesian closed, multiple category. 
and the cocompleteness properties of $S$; if $S$ is to be as cocomplete as $\mathbb{Q}$ then every admissible category must be an ordered object.

To illustrate the fact that an internal full subcategory $S$ of $\mathscr{Q}$ gives a good notion of size for categories in $\mathcal{Q}$, it is shown in $\$ 6$ that the 2-category $\operatorname{Cat}(\mathcal{Q})$ inherits a Yoneda structure (Street-Walters [30]) with $\mathscr{P} A=\left[A^{\text {op }}, S\right]$ when $\mathbb{Q}$ is cartesian closed. Indeed, this structure arises from a fibrational cosmos (= "cosmos" in the sense of Street [28]).

This paper investigates the existence and nature of internal full subcategories, especially in cartesian closed, locally presentable categories $\mathbb{Q}$. It is shown in $\$ 7$ that internal full subcategories of [ $\bigodot^{\text {op }}$, Set] essentially amount to full subfunctors of $\left[\left(\mathcal{\downarrow} \downarrow^{\text {op }}\right.\right.$, Set] which actually land in Cat. These include Grothendieck topolo-

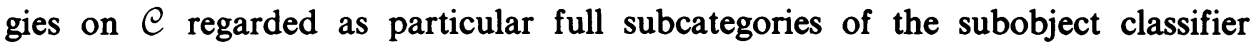
$\left[\left(C_{\downarrow}-1\right)^{\text {op }}, 2\right]$ of [ $\bigodot^{\text {op }}$, Set], and include "calibrations" of $\mathcal{C}$ in the sense of Bénabou [3]. Cosmos theory arising from the latter is shown in $\$ 9$ to lead to the theory of locally internal categories (see Johnstone [18, Appendix]); locally internal categories are essentially the admissible categories in [ $\complement^{\text {op }}$, Set] relative to the maximum calibration of $C$.

In fact, internal full subcategories of [ $\bigodot^{\text {op }}$, Set] can be identified with a certain class of Gabriel theories (3.5) on $C$; namely those which are pullback stable. These are more general than Grothendieck topologies on $\mathcal{C}$ in that they allow cocones in

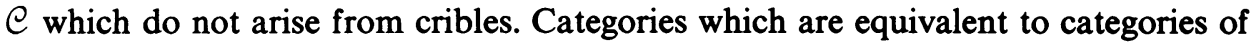
models for pullback stable Gabriel theories are precisely internally complete (7.24), locally presentable (3.4) categories (see Theorem (7.25)). The category of sets with distinguished subsets is such without being a topos.

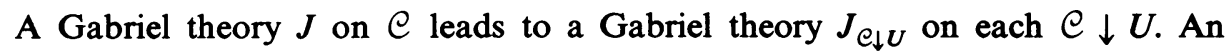
internal full subcategory of a locally presentable category $\operatorname{Mod}(J$, Set) is shown in $\$ 8$ to amount essentially to a full subfunctor of $\operatorname{Mod}\left(J_{\mathcal{L}_{\downarrow}-}\right.$, Set) which not only lands in Cat but is also a model for $J$. This result is applied to the category $r$-tplcat of $r$-tple categories for each $r$ to produce internal full subcategories of $(r+1)$ dimensional cubes. These give the ingredients for a comprehension scheme at each level of the hierarchy of Gray [17].

Our basic notation is that of Mac Lane [21] and Kelly-Street [19]. We write $[\mathbb{Q}, \mathscr{B}]$ for the category of functors from $\mathscr{Q}$ to $\mathscr{B}$ (rather than $\mathscr{B}^{\mathbb{Q}}$ ). For a 2-category $\mathscr{K}$, we write $|\mathcal{K}|$ for the underlying category (rather than $\mathcal{K}_{0}$ which has simplicial overtones).

This work represents a substantial revision and extension of a preprint by the same title circulated in January 1976. Some of the material herein has been exposed in seminars at the University of Sussex (July 1976) and Columbia University (October 1976, February 1977). Partial support was provided by a grant from the National Science Foundation of the United States (1976-1977) which enabled the author to spend his study leave at Wesleyan University (Middletown, Connecticut).

1. The Grothendieck construction. For an ordered pair $A, B$ of categories there is a functor $-\downarrow B \times A \downarrow-: A \times B^{\text {op }} \rightarrow$ Cat whose value at $(a, b)$ is the product of the two comma categories $b \downarrow B, A \downarrow a$ and which is given on arrows by composition. 
For a 2-category $\mathscr{K}$, the Grothendieck construction $\mathcal{G}(F)$ (or more precisely, $\mathcal{G}_{B}(F)$ ) on a functor $F: A^{\text {op }} \times B \rightarrow \mathcal{K}$ is the $(-\downarrow B \times A \downarrow-)$-indexed colimit $\operatorname{col}(-\downarrow B \times$ $A \downarrow-, F)$ of $F$ in the sense of Street [29]. This means that there is an isomorphism of categories

$$
\mathscr{K}(\mathcal{G}(F), X) \cong\left[A \times B^{\text {op }}, \text { Cat }\right](-\downarrow B \times A \downarrow-, \mathcal{K}(F, X))
$$

which is 2-natural in $X$.

The particular case of interest here is where $\mathscr{K}=$ Cat. Then (1.1) amounts to a 2-natural isomorphism

$$
[\mathcal{G}(F), X] \cong\left[A^{\text {op }} \times B, \text { Cat }\right](F,[-\downarrow B \times A \downarrow-, X]) .
$$

In other words, $\mathcal{G}$ is a left adjoint for the 2 -functor

$$
[-\downarrow B \times A \downarrow-, \sim]: \text { Cat } \rightarrow\left[A^{\text {op }} \times B \text {, Cat }\right] \text {. }
$$

The existence of $\mathcal{G}$ follows from the cocompleteness of the 2-category Cat. However, the presence of the objects $1_{a}, 1_{b}$ in $A \downarrow a, b \downarrow B$ means that there are canonical choices of representatives for equivalence classes in $\amalg_{a, b} b \downarrow B \times A \downarrow a$ $\times F(a, b)$, and we obtain the following simple description of the category $\mathcal{G}(F)$ (compare Gray [17, pp. 267-271]).

(1.4) The objects are triples $(a, x, b)$ where $a, b, x$ are objects of $A, B, F(a, b)$, respectively. An arrow $(\alpha, \xi, \beta):(a, x, b) \rightarrow\left(a^{\prime}, x^{\prime}, b^{\prime}\right)$ consists of arrows $\alpha: a \rightarrow a^{\prime}$, $\beta: b \rightarrow b^{\prime}, \xi: F(a, \beta) x \rightarrow F\left(\alpha, b^{\prime}\right) x^{\prime}$ in $A, B, F\left(a, b^{\prime}\right)$, respectively. Composition is given by

$$
\left(\alpha^{\prime}, \xi^{\prime}, \beta^{\prime}\right)(\alpha, \xi, \beta)=\left(\alpha^{\prime} \alpha, F\left(\alpha, b^{\prime \prime}\right) \xi^{\prime} \cdot F\left(a, \beta^{\prime}\right) \xi, \beta^{\prime} \beta\right) .
$$

To complete the definition of $\mathcal{G}:\left[A^{\text {op }} \times B\right.$, Cat $] \rightarrow$ Cat as a 2-functor notice that a natural transformation $\sigma: F \rightarrow G$ determines a functor $\mathcal{G}(\sigma): \mathcal{G}(F) \rightarrow \mathcal{G}(G)$ via the equations:

$$
\begin{aligned}
& \mathcal{G}(\sigma)(a, x, b)=\left(a, \sigma_{a, b}(x), b\right), \\
& \mathcal{G}(\sigma)(\alpha, \xi, \beta)=\left(\alpha, \sigma_{a, b^{\prime}}(\xi), \beta\right) ;
\end{aligned}
$$

and a modification $m: \sigma \rightarrow \tau$ determines a natural transformation $\mathcal{S}(m): \mathcal{G}(\sigma) \rightarrow$ $\mathcal{G}(\tau)$ with $\mathcal{S}(m)_{(a, x, b)}=\left(1_{a}, m_{a b}(x), 1_{b}\right)$.

The above description makes it clear that $\mathcal{G}$ lifts to a 2-functor

$$
\mathcal{G}:\left[A^{\text {op }} \times B, \text { Cat }\right] \rightarrow \text { Cat } \downarrow B \times A
$$

where we regard $\mathcal{G}(F)$ as a category over $B \times A$ via the projection which takes $(\alpha, \xi, \beta)$ to $(\beta, \alpha)$.

(1.6) The 2-functor (1.5) is faithful and locally fully faithful (the latter means it induces fully faithful functors on hom-categories).

(1.7) The 2-functor (1.5) has a left adjoint $\Re$ whose value at $\left(\begin{array}{c}q \\ p\end{array}\right): M \rightarrow B \times A$ is the functor $\mathscr{T}(M): A^{\text {op }} \times B \rightarrow$ Cat described as follows. The objects of $\mathfrak{N}(M)(a, b)$ are triples $(\alpha, m, \beta)$ where $m$ is an object of $M$ and $\alpha: a \rightarrow p m, \beta$ : $q m \rightarrow b$ are arrows of $A, B$, respectively. An arrow $\mu:(\alpha, m, \beta) \rightarrow\left(\alpha^{\prime}, m^{\prime}, \beta^{\prime}\right)$ in $\mathfrak{N}(M)(a, b)$ is an arrow $\mu: m \rightarrow m^{\prime}$ in $M$ such that $\alpha^{\prime}=p \mu \cdot \alpha, \beta^{\prime} \cdot q \mu=\beta$. Composition is that of $M$. For $(\theta, \phi):(a, b) \rightarrow(c, d)$ in $A^{\text {op }} \times B$, the functor $\mathfrak{N}(M)(\theta, \phi): \mathfrak{R}(M)(a, b) \rightarrow \mathfrak{N}(M)(c, d)$ is given by 


$$
\Re(M)(\theta, \phi)(\alpha, m, \beta)=(\alpha \theta, m, \phi \beta), \quad \Re(M)(\theta, \phi) \mu=\mu .
$$

(1.8) The 2-functor (1.5) is monadic. This means that the 2-functor induced by $\mathcal{G}$ from [ $A^{\text {op }} \times B$, Cat] to the 2-category $\operatorname{Spl}(B, A)$ of Eilenberg-Moore algebras for the monad $\mathcal{G} \mathfrak{N}$ on Cat $\downarrow B \times A$ generated by the adjunction $\mathfrak{R} \dashv \mathcal{G}$, is an equivalence. So $\mathcal{G}$ induces an equivalence of 2-categories

$$
\left[A^{\text {op }} \times B, \text { Cat }\right] \simeq \operatorname{Spl}(B, A) .
$$

(1.10) The objects of $\operatorname{Spl}(B, A)$ are called split fibrations from $B$ to $A$. Any two $\mathcal{G} \mathfrak{N}$-algebra structures on an object of $\operatorname{Cat} \downarrow B \times A$ are isomorphic, so $\operatorname{Spl}(B, A)$ is equivalent to a locally full sub-2-category of Cat $\downarrow B \times A$.

(1.11) An object of a 2-category $\mathcal{K}$ is called discrete when all 2-cells between arrows into that object are identities. Consequently, the full sub-2-category $D \mathscr{K}$ of discrete objects in $\mathcal{K}$ is a mere category; all its 2-cells are identities. Discreteness is preserved by 2 -functors with left adjoints; in particular, by equivalences.

One sees immediately that $D\left[A^{\mathrm{op}} \times B\right.$, Cat $] \cong\left[A^{\mathrm{op}} \times B\right.$, Set $]$, so $\mathcal{G}$ induces an equivalence of categories

$$
\left[A^{\text {op }} \times B, \text { Set }\right] \simeq D \operatorname{Spl}(B, A) .
$$

(1.13) Objects of $D \operatorname{Spl}(B, A)$ are called discrete fibrations from $B$ to $A$. An object $\left(\begin{array}{l}q \\ p\end{array}\right): M \rightarrow B \times A$ of Cat $\downarrow B \times A$ is discrete if and only if $p \mu, q \mu$ are both identities implies $\mu$ is an identity for all arrows $\mu$ in $M$. Discrete fibrations from $B$ to $A$ are precisely split fibrations which are discrete objects of Cat $\downarrow B \times A$. A discrete object of Cat $\downarrow B \times A$ admits at most one $\mathcal{G} \mathfrak{N}$-algebra structure. The category $D \operatorname{Spl}(B, A)$ is a full sub-2-category of Cat $\downarrow B \times A$.

(1.14) In view of the composite equivalence

$$
\operatorname{Spl}(B, A) \simeq\left[A^{\text {op }} \times B, \text { Cat }\right] \simeq\left[1^{\text {op }} \times\left(A^{\text {op }} \times B\right), \text { Cat }\right] \simeq \operatorname{Spl}\left(A^{\text {op }} \times B, 1\right),
$$

the reader may wonder why we have chosen to describe a two-sided Grothendieck construction. The reason is that, for a functor $F: A^{\mathrm{op}} \times B \rightarrow$ Cat, the categories $\mathcal{G}_{B}^{A}(F)$ and $\mathcal{G}_{A^{\infty \times} \times B}^{1}(F)$, while having the same objects, are structurally different. The relationship (and hence also the above equivalence) is not "2-canonical"; it involves breaking categories up into sets of objects and sets of arrows.

(1.15) We next consider the question of "naturality" of the 2-functors

$$
\mathcal{G}_{B}^{A}:\left[A^{\text {op }} \times B, \text { Cat }\right] \rightarrow \text { Cat } \downarrow B \times A
$$

in $A, B$. The assignment $B, A \mapsto\left[A^{\text {op }} \times B\right.$, Cat $]$ can be extended to arrows and 2-cells by composition to define a 2 -functor

$$
\left[(-)^{\text {op }} \times \sim, \text { Cat }\right]: \text { Cat }^{\text {op }} \times \text { Cat }^{\text {coop }} \rightarrow \text { 2-CAT. }
$$

The assignment $B, A \mapsto$ Cat $\downarrow B \times A$ can be extended to arrows by pullback to define a pseudo-functor

$$
\text { Cat } \downarrow \sim \times-: \mid \text { Cat }\left.\right|^{\text {op }} \times \mid \text { Cat }\left.\right|^{\text {op }} \rightarrow 2 \text {-CAT }
$$

(for the terminology see Kelly-Street [19]); there is no natural extension of the assignment to 2-cells. It is readily checked that $\mathcal{G}_{B}^{A}$ are the components of a 
pseudo-natural transformation

$$
\mathcal{G}_{\sim}^{-}:\left[(-)^{\text {op }} \times \sim, \text { Cat }\right] \rightarrow \text { Cat } \downarrow \sim \times-
$$

between pseudo-functors from $\mid$ Cat $\left.\right|^{\text {op }} \times \mid$ Cat $\left.\right|^{\text {op }}$ to 2-CAT.

(1.18) From (1.16), (1.9) we can extend the assignment $B, A \mapsto \operatorname{Spl}(B, A)$ to a pseudo-functor

$$
\operatorname{Spl}(\sim,-): \text { Cat }^{\text {op }} \times \text { Cat }^{\text {coop }} \rightarrow \text { 2-CAT }
$$

such that the equivalences (1.9) are pseudo-natural. The forgetful 2-functors $\operatorname{Spl}(B, A) \rightarrow$ Cat $\downarrow B \times A$ are the components of a pseudo-natural transformation from $\operatorname{Spl}(\sim,-)$ to Cat $\downarrow \sim \times-$ as pseudo-functors from $\mid$ Cat $\left.\right|^{\text {op }} \times \mid$ Cat $\left.\right|^{\text {op }}$ to 2 CAT.

(1.20) Since discreteness is preserved by pulling back, the assignment $B, A \mapsto$ $D \operatorname{Spl}(B, A)$ extends to a pseudo-functor

$$
D \operatorname{Spl}(\sim,-): \mathrm{Cat}^{\mathrm{op}} \times \mathrm{Cat}^{\mathrm{coop}} \rightarrow \mathrm{CAT}
$$

which is a sub-pseudo-functor of (1.19).

(1.21) Note that from (1.9), (1.12) there are pseudo-natural equivalences

$$
\begin{gathered}
\operatorname{Spl}(B, A) \simeq \operatorname{Spl}\left(A^{\text {op }}, B^{\text {op }}\right), \\
D \operatorname{Spl}(B, A) \simeq D \operatorname{Spl}\left(A^{\text {op }}, B^{\text {op }}\right) .
\end{gathered}
$$

2. Fibrational cosmoi. The monad $\mathcal{G} \Re$ on $\operatorname{Cat} \downarrow B \times A(1.8)$ can be generalized to the case where $A, B$ are objects of a finitely complete 2-category $\mathcal{K}$.

(2.1) For objects $A, B$ of a finitely complete (see Street [29]) 2-category $\mathscr{K}$, we shall describe a monad $\mathcal{T}$ (or more strictly $\mathcal{T}_{B}^{A}$ ) on the 2-category $\mathscr{K} \downarrow B \times A$. The 2-functor $\mathcal{T}: \mathscr{K} \downarrow B \times A \rightarrow \mathcal{K} \downarrow B \times A$ is described as follows. For an object $\left(\begin{array}{c}q \\ p\end{array}\right)$ : $M \rightarrow B \times A$ over $B \times A$, the object $\mathcal{T}(M)$ over $B \times A$ is the limit of the diagram

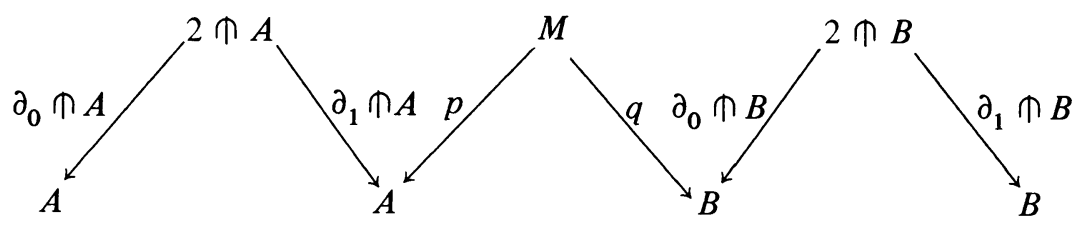

with arrow into $B \times A$ induced by the projections from $\mathscr{T}(M)$ to the outside $A, B$. The 2-functoriality of the diagram (2.2) induces 2-functoriality for the limits. The unique functor $\iota: 2 \rightarrow 1$ and the functor $\partial_{1}: 2 \rightarrow 3$ given by $\partial_{1}(0)=0, \partial_{1}(1)=2$, induce arrows $\iota \pitchfork A: A \rightarrow \mathbf{2} \pitchfork A, \iota \pitchfork B: B \rightarrow 2 \pitchfork B$, and $\partial_{1} \pitchfork A: 3 \pitchfork A \rightarrow 2 \pitchfork A$, $\partial_{1} \pitchfork B: 3 \pitchfork B \rightarrow 2 \pitchfork B$. The components of the unit of the monad are the arrows $M \rightarrow \mathcal{T}(M)$ induced by $\iota \pitchfork A, \iota \pitchfork B$. The components of the multiplication of the monad are the arrows $\mathcal{T} \mathcal{T}(M) \rightarrow \mathcal{T}(M)$ induced by $\partial_{1} \pitchfork A, \partial_{1} \pitchfork B$.

(2.3) Write $\operatorname{Spl}(B, A)$ (or more precisely, $\operatorname{Spl}(B, A ; \mathcal{K})$ ) for the 2-category of Eilenberg-Moore algebras for the monad $\mathcal{T}_{B}^{A}$ on $\mathcal{K}_{\downarrow} B \times A$. The objects of $\operatorname{Spl}(B, A)$ are called split fibrations from $B$ to $A$ in $\mathcal{K}$. Any two $\mathcal{T}$-algebra structures on an object of $\mathscr{K} \downarrow B \times A$ are isomorphic, so $\operatorname{Spl}(B, A)$ is equivalent to a locally full sub-2-category of $\mathscr{K} \downarrow B \times A$. 
(2.4) For an object $M$ over $B \times A$ and arrows $a: A^{\prime} \rightarrow A, b: B^{\prime} \rightarrow B$ in $\mathscr{K}$, we write $M(a, b)$ for the object over $B^{\prime} \times A^{\prime}$ obtained by pullback along $b \times a$. We call $M(a, b)$ the fibre of $M$ over $a, b$. In particular, when $A=B, 2 \pitchfork A(a, b)$ is the comma object of $a, b$ and denoted by $a \downarrow b$.

(2.5) A structure of $\mathcal{T}$-algebra on an object $E$ over $B \times A$ amounts precisely to a structure of $\mathcal{G} \mathscr{T}$-algebra on each category $\mathcal{K}(X, E)$ over $\mathcal{K}(X, B) \times \mathcal{K}(X, A)$ 2-naturally in $X$. This follows from the Yoneda lemma and the fact that $\mathcal{T}$ is defined purely in terms of limits in $\mathcal{K}$ and is taken into $\mathcal{G} \mathscr{K}$ by representables $\mathcal{K}(X,-)$.

(2.6) From (1.17), (1.19), (2.5) it follows that a structure of $\mathcal{T}$-algebra on an object $E$ over $B \times A$ induces a structure of $\mathcal{T}$-algebra on any fibre $E(a, b)(2.4)$. Indeed, we obtain a pseudo-functor

$$
\operatorname{Spl}(\sim,-): \mathcal{K}^{\text {op }} \times \mathcal{K}^{\text {coop }} \rightarrow \text { 2-CAT }
$$

whose value at $B, A$ is $\operatorname{Spl}(B, A)$ and at $b, a$ is pullback along $b \times a$.

(2.8) Each split fibration $E$ from $B$ to $A$ gives rise to a functor

$$
E(-, \sim): \mathscr{K}\left(A^{\prime}, A\right)^{\text {op }} \times \mathscr{K}\left(B^{\prime}, B\right) \rightarrow \operatorname{Spl}\left(B^{\prime}, A^{\prime}\right)
$$

whose value at $a, b$ is $E(a, b)$ and whose value at $\alpha: a^{\prime} \rightarrow a, \beta: b \rightarrow b^{\prime}$ is $E(\alpha, \beta)=\operatorname{Spl}(\beta, \alpha)_{E}: \operatorname{Spl}(b, a) E \rightarrow \operatorname{Spl}\left(b^{\prime}, a^{\prime}\right) E$.

(2.9) Objects of $\operatorname{DSpl}(B, A)(1.11)$ are called discrete fibrations from $B$ to $A$ in $\mathcal{K}$. It follows from (1.13), (2.5) that $\operatorname{DSpl}(B, A)$ is a full sub-2-category of $\mathscr{K} \downarrow B \times A$. There is a sub-pseudo-functor

$$
\operatorname{DSpl}(\sim,-): \mathscr{K}^{\text {op }} \times \mathcal{K}^{\text {coop }} \rightarrow \mathrm{CAT}
$$

of (2.7). For a discrete fibration $E$ from $B$ to $A$, the functor (2.8) factors through a functor:

$$
E(-, \sim): \mathscr{K}\left(A^{\prime}, A\right)^{\text {op }} \times \mathscr{K}\left(B^{\prime}, B\right) \rightarrow \operatorname{DSpl}\left(B^{\prime}, A^{\prime}\right) .
$$

(2.10) When $\mathscr{K}=$ Cat and $A^{\prime}=B^{\prime}=1$, notice that $\operatorname{Spl}\left(B^{\prime}, A^{\prime}\right)=$ Cat, and, for any functor $F: A^{\text {op }} \times B \rightarrow$ Cat, the functor

$$
(\mathcal{G F})(-, \sim):[1, A]^{\mathrm{op}} \times[1, B] \rightarrow \text { Cat }
$$

of (2.8) with $E=\mathcal{G} F$ is isomorphic to $F$.

(2.11) Definition. A fibrational cosmos consists of the following data:

(a) a finitely complete 2-category $\mathcal{K}$;

(b) a 2-functor $\mathscr{P}: \mathscr{K}^{\text {coop }} \rightarrow \mathcal{K}$ with a left adjoint $\mathscr{P} *: \mathscr{K} \rightarrow \mathscr{K}^{\text {coop }}$;

(c) for each object $A$ of $\mathcal{K}$, a discrete fibration $\in_{A}$ from $\mathscr{P} A$ to $A$ in $\mathscr{K}$; satisfying the following axioms:

(i) for each $A, B$, the functor

$$
\in_{A}(A, \sim): \mathscr{H}(B, \mathscr{P} A) \rightarrow \operatorname{DSpl}(B, A)
$$

(see (2.9)) is fully faithful;

(ii) for each $B$, the functors of (i) are the components of a pseudo-natural transformation

$$
\epsilon_{-}(-, \sim): \mathscr{K}(B, \mathscr{P}-) \rightarrow \operatorname{DSpl}(B,-)
$$

between pseudo-functors $\mathcal{F}^{\text {coop }} \rightarrow$ CAT. 
(2.12) A fibrational cosmos amounts precisely to what was called a "cosmos" in Street [28], except that we have here insisted on a terminal object in $\mathscr{K}$. It was shown in [28] that a large portion of category theory could be developed elementarily in a fibrational cosmos.

(2.13) For any object $A$, the object $2 \pitchfork A$ over $A \times A$ is a discrete fibration from $A$ to $A$. An arrow $a: A^{\prime} \times A$ is called admissible when there exist an arrow $h$ : $A \rightarrow \mathscr{P} A^{\prime}$ and an isomorphism $\varepsilon_{A^{\prime}}\left(A^{\prime}, h\right) \simeq 2 \pitchfork A(a, A)(=a \downarrow A)$ over $A \times A^{\prime}$; the arrow $h$ is unique up to isomorphism and denoted by $\operatorname{hom}_{A}(a, 1)$. An object $A$ is called admissible (or legitimate, or locally small) when 1: $A \rightarrow A$ is admissible; in this case $\operatorname{hom}_{A}(1,1)$ is denoted by $y_{A}: A \rightarrow \mathcal{P}_{A}$ and called the yoneda arrow of $A$. Also $\in_{A} \simeq(2 \pitchfork \mathscr{P} A)\left(y_{A}, \mathscr{P} A\right)$, so $y_{A}$ is admissible and hom $\mathscr{P}_{A}\left(y_{A}, 1\right) \simeq 1_{\mathscr{P} A}$. If $A$ and $f: A \rightarrow B$ are admissible there is a 2-cell

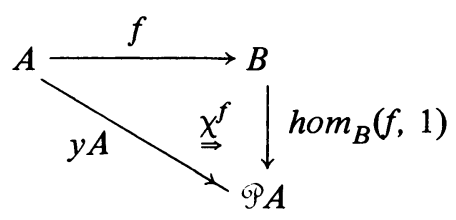

which is defined by the condition that $\in_{A}\left(A, \chi^{f}\right)$ is isomorphic to the canonical arrow $2 \pitchfork A \rightarrow(2 \pitchfork B)(f, f)$ over $A \times A$.

(2.15) A fibrational cosmos structure on $\mathscr{K}$ gives rise to a Yoneda structure on $\mathscr{K}$ in the sense of Street-Walters [30]; indeed their Axiom 3* is satisfied. The data for the Yoneda structure are the admissible arrows described in (2.13) and the diagrams (2.14).

\section{Locally presentable categories.}

(3.1) Suppose $\alpha$ is a regular cardinal. A category $g$ is called $\alpha$-filtered when, for each functor $K: \mathcal{L} \rightarrow \mathcal{G}$ where the set of arrows of $\mathcal{L}$ has cardinality $<\alpha$, there exist an object $X$ of $\mathcal{G}$ and a cocone $K \Rightarrow X$. An $\alpha$-filtered colimit is a colimit of a functor whose source is an $\alpha$-filtered category.

(3.2) An object $A$ of a category $\mathcal{Q}$ is called $\alpha$-presentable when the representable functor $Q(A,-)$ preserves $\alpha$-filtered colimits.

(3.3) A set $\mathcal{G}$ of objects of a category $Q$ is called strongly generating when, for all arrows $f: A \rightarrow B$ in $\mathbb{Q}$, if $\mathscr{Q}(G, f): \mathcal{Q}(G, A) \rightarrow \mathscr{Q}(G, B)$ is an isomorphism for all $G \in \mathcal{G}$ then $f$ is an isomorphism.

(3.4) A category is called locally presentable when it satisfies the following conditions:

(i) it is small cocomplete and finitely complete;

(ii) it has small homsets;

(iii) there exist a small regular cardinal $\alpha$ and a small strongly generating set of $\alpha$-presentable objects.

(3.5) A Gabriel theory $J$ on a category $\mathcal{C}$ is a function which assigns to each object $U$ of $\mathcal{C}$ a small set $J(U)$ whose elements are natural transformations 


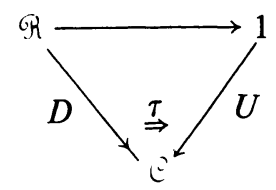

(that is, cocones with vertex $U$ ) where $R$ is small.

(3.6) A model of $J$ in a category $\mathcal{X}$ is a functor $F$ : $\mathcal{C}^{\text {op }} \rightarrow \mathfrak{X}$ such that, for each object $U$ of $\mathcal{C}$ and each $\tau \in J(U)$, the cone $F \tau: F U \Rightarrow F D$ is a limit for $F D$. Write $\operatorname{Mod}(J, \mathfrak{X})$ for the full subcategory of $\left[\complement^{o p}, \mathfrak{X}\right.$ ] consisting of the models of $J$. One easily verifies that $\operatorname{Mod}(J, \mathfrak{X})$ is closed under pointwise limits in [ $\mathcal{C}^{\circ}, \mathfrak{X}$ ].

Proofs of the following two theorems can be found in Gabriel-Ulmer [15].

(3.7) THEOREM. A category $\mathscr{Q}$ is locally presentable if and only if there exist a small category $\mathcal{C}$, a Gabriel theory $J$ on $\mathcal{C}$, and an equivalence of categories $\mathbb{Q} \simeq$ $\operatorname{Mod}(J$, Set).

(3.8) THEOREM. If $J$ is a Gabriel theory on a small category $\mathcal{C}$ and $\mathcal{X}$ is locally presentable then $\operatorname{Mod}(J, \mathfrak{X})$ is locally presentable and its inclusion in [£op, $\mathfrak{X}$ ] has a left adjoint.

(3.9) Our interest here is mainly in cartesian closed locally presentable categories. The following theorem is essentially a corollary of the Reflection Theorem (Theorem 1.2 and Corollary 2.1) of Day [6]. (It is a little stronger than the statement at the top of p. 4 of Day [6].)

(3.10) THEOREM. Suppose $\mathfrak{T}$ is a full subcategory of a cartesian closed category $\mathscr{P}$ and that the inclusion has a left adjoint L. Properties (a), (b) below are equivalent and imply property (c). If $\mathfrak{T}$ is strongly generating (3.3) for $\mathcal{P}$ then (c) implies (b).

(a) L preserves finite products.

(b) For all $P \in \mathcal{P}, F \in \mathfrak{N}$, there is an isomorph in $\mathfrak{R}$ of the internal hom $[P, F]$ in $\mathcal{P}$.

(c) $\mathfrak{N}$ is cartesian closed.

Proof. Our (a), (b) amount to (8), (1) of Day's Reflection Theorem and so are equivalent. Under condition (b) the restriction of the internal hom of $\mathscr{P}$ to $\mathfrak{N}$ yields (c). Finally assume (c), and let $[F, G]$ be the internal hom of $F$, $G$ in $\Re$. Evaluation $[F, G\rfloor \times F \rightarrow G$ induces an arrow $\llbracket F, G\rfloor \rightarrow[F, G]$ which, for $H \in \mathfrak{T}$, induces the composite isomorphism:

$$
\mathscr{P}(H, \llbracket F, G \rrbracket) \cong \mathscr{T}(H, \llbracket F, G \rrbracket) \cong \mathscr{P}(H \times F, G) \simeq \mathscr{P}(H,[F, G])
$$

If $\mathscr{N}$ is strongly generating this implies $[F, G] \simeq \llbracket F, G \rrbracket \in \mathscr{R}$. It follows that the component $[F, G] \rightarrow L[F, G]$ of the unit of the adjunction is an isomorphism. Again using that $\mathfrak{N}$ is strongly generating we see that we are in the situation of (2) of Day's Reflection Theorem; so his (1) holds which is our (b). 
(3.11) Theorem. Suppose $J$ is a Gabriel theory on a small (respectively, finite) category $\mathcal{C}$ such that the representable functors are models. The following six conditions are equivalent.

(a) $\operatorname{Mod}(J$, Set) is cartesian closed;

(b) for all $F, P \in\left[e^{o p}\right.$, Set], if $F$ is a model of $J$ then $[P, F]$ is a model of $J$;

(c) the left adjoint of the inclusion of $\operatorname{Mod}(J$, Set) in [@op, Set] is finite product preserving;

(d) for all small (respectively, finitely) complete, cartesian closed, locally small categories $\mathbb{Q}$, the category $\operatorname{Mod}(J, \mathbb{Q})$ is cartesian closed;

(e) for all categories $\mathbb{Q}$ as in (d) and $F, P \in\left[\bigodot^{\circ p}, \mathbb{Q}\right]$, if $F$ is a model of $J$ then $[P, F]$ is a model of $J$;

(f) for all cartesian closed, locally presentable categories $\mathbb{Q}$, the left adjoint to the inclusion of $\operatorname{Mod}(J, \mathbb{Q})$ in $\left[\mathfrak{C}^{\circ}, \mathbb{Q}\right]$ is finite product preserving. ${ }^{1}$

Proof. The equivalence of (a), (b), (c) will follow from (3.10) once we know that $\operatorname{Mod}\left(J\right.$, Set) is strongly generating in [ $\bigodot^{\text {op }}$, Set]. But the representables are dense in

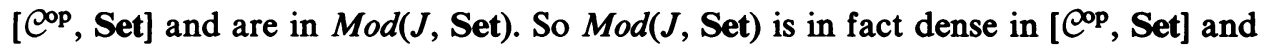
hence strongly generating.

Clearly (f) $\Rightarrow$ (c) and (d) $\Rightarrow$ (a). By (3.10), (e) $\Rightarrow$ (f) and (e) $\Rightarrow(d)$. We complete the proof by showing that $(\mathrm{b}) \Rightarrow(\mathrm{e})$. Note that $Q: \mathcal{C}^{\circ} \rightarrow \mathbb{Q}$ is a model for $J$ if and only if, for all $A$ of $\mathbb{Q}, \mathbb{Q}(A, Q)$ is a model of $J$ in Set. The internal hom in [ ${ }^{\text {op }}, \mathbb{Q}$ ] is given by the end formula:

$$
[P, Q]=\int_{V}[P V, \mathcal{C}(V, U) \pitchfork Q V]
$$

Take $P, F: \mathcal{C}^{\text {op }} \rightarrow \mathbb{Q}$ where $F$ is a model of $J$ in $\mathcal{Q}$. We have isomorphisms:

$$
\begin{aligned}
\mathscr{Q}(A,[P, F]) & =\mathbb{Q}\left(A, \int_{V}[P V, \mathcal{C}(V,-) \pitchfork F V]\right) \\
& \cong \int_{V} \mathscr{Q}(A \times P V, \mathcal{C}(V,-) \pitchfork F V) \\
& \cong \int_{V} \int_{B} \operatorname{Set}(\mathscr{Q}(B, A \times P V), \mathcal{Q}(B, \mathcal{C}(V,-) \pitchfork F V)) \\
& \cong \int_{B} \int_{V} \operatorname{Set}(\mathscr{Q}(B, A \times P V), \operatorname{Set}(\mathcal{C}(V,-), \mathcal{Q}(B, F V))) \\
& \cong \int_{B}[\mathcal{Q}(B, A \times P), \mathscr{Q}(B, F)],
\end{aligned}
$$

and the last of these functors is a model in Set by (b). So $[P, F]$ is a model in $\mathcal{Q}$.

(3.12) Let $\Delta_{+}$denote the full subcategory of $\mid$Cat $\mid$consisting of the nonempty finite ordinals. Under composition $\Delta_{+}$is generated by the cosimplicial diagram consisting of the monomorphisms $\partial_{m}: \mathbf{n} \rightarrow \mathbf{n}+1$ for $0<m<n$ where $\partial_{m}<\partial_{m+1}$, and the epimorphisms $\iota_{m}: \mathbf{n}+1 \rightarrow \mathbf{n}$ for $0<m<n-1$ where $\partial_{m} \dashv \iota_{m} \dashv \partial_{m+1}$ in Cat.

\footnotetext{
'Some of the implications of this theorem appear in [2].
} 
(3.13) The inclusion $\Delta_{+} \rightarrow \mid$ Cat $\mid$ induces a "singular" functor $I: \mid$ Cat $\mid \rightarrow\left[\Delta_{+}^{\text {op }}\right.$, Set $]$ whose value at $A$ is $I A=[-, A]: \Delta_{+}^{\text {op }} \rightarrow$ Set.

(3.14) On the other hand, we also have a functor $\Delta_{+}^{\text {op }} \rightarrow[\mid$ Cat $\mid$, Set $]$ whose value at $\mathbf{n}$ is [n, -]. In other words, we have a simplicial object in [|Cat|, Set] which we denote by:

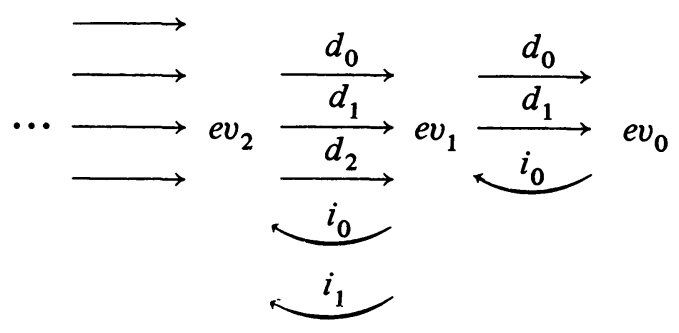

For $n>0$ and a category $A$, the elements of the set $A_{n}=e v_{n} A=(I A)(\mathrm{n}+1)=[\mathrm{n}$ $+1, A]$ are composable $n$-tuples of arrows in the category $A$; also $d_{n} A=\left[\partial_{m}, A\right]$. For a functor $f: A \rightarrow B$, put $f_{n}=e v_{n} f=(I f)_{n+1}: A_{n} \rightarrow B_{n}$.

(3.15) It follows that $I: \mid$ Cat $\mid \rightarrow\left[\Delta_{+}^{\text {op }}\right.$, Set $]$ is fully faithful. Since $\mid$ Cat $\mid$ is small cocomplete, $I$ has a left adjoint $L:\left[\Delta_{+}^{\text {op }}\right.$, Set $] \rightarrow \mid$ Cat $\mid$ whose value at a simplicial set $F$ is the category:

$$
L(F)=\int^{\mathbf{n}} F \mathbf{n} \times \mathbf{n} .
$$

An alternative description given by Gabriel-Zisman [14, p. 33] is: form the free category on the graph

$$
F 2 \underset{F \partial_{1}}{\stackrel{F \partial_{0}}{\rightarrow}} F 1
$$

and factor out by the equivalence relation generated by the relations:

$$
\begin{aligned}
& \left(F l_{0}\right) x \sim 1_{x} \text { for all } x \in F 1, \\
& \left(F \partial_{1}\right) z \sim\left(F \partial_{2}\right) z \cdot\left(F \partial_{0}\right) z \text { for all } z \in F 3 .
\end{aligned}
$$

(3.16) Let $J_{c a t}$ denote the Gabriel theory on $\Delta_{+}$for which $J_{c a t}(1)$ and $J_{c a t}(2)$ are empty and, for $n>2, J_{c a t}(n+1)$ consists of the single cocone:

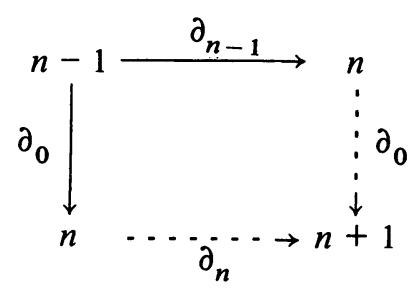

Notice that the above cocones are pushouts in Cat and hence in $\Delta_{+}$; so $J_{c a t}$ has the property that the representables are models.

(3.17) It is easily seen that $I$ (3.13) induces an equivalence of categories

$$
\mid \text { Cat } \mid \simeq \operatorname{Mod}\left(J_{\text {cal }}, \text { Set }\right) \text {. }
$$


Since |Cat| is cartesian closed, we have the following corollary of Theorem (3.11).

(3.18) THEOREM. The functor $L:\left[\Delta_{+}^{\text {op }}\right.$, Set $] \rightarrow \mid$ Cat $\mid$ of $(3.15)$ is finite product preserving.

(3.19) The observation (3.18) seems to have been missed by Gabriel-Zisman [14] since they invoke the Eilenberg-Zilber lemma (p. 26) and arguments involving "shuffles" to prove that the left adjoint to the inclusion of the category $|\mathbf{G p d}|$ of groupoids in $\left[\Delta_{+}^{\text {op }}\right.$, Set] is finite product preserving. However, if $P$ is a category and $F$ is a groupoid it is clear that the category $[P, F]$ is a groupoid, so, by (3.10), the left adjoint of the inclusion of $|\mathbf{G p d}|$ in $\mid$ Cat $\mid$ preserves finite products. The same argument can be applied to the category |Ord| of partially ordered sets. Combining this with (3.18) we obtain:

(3.20) COROLlaRY. The functors $\mid$ Gpd $\mid \rightarrow\left[\Delta_{+}^{\mathrm{op}}\right.$, Set $]$, |Ord $\mid \rightarrow\left[\Delta_{+}^{\mathrm{op}}\right.$, Set $]$ obtained by restriction of $I$ (3.13) both have finite-product-preserving left adjoints.

(3.21) There are two variants of (3.12) to (3.20) each with its uses. The category $\Delta_{+}$could be replaced by the full subcategory of $\mid$Cat $\mid$consisting of the ordinals $1,2,3,4$. The simplicial objects are truncated; however, all the results remain true including (3.18). This variant is essential for those to whom "small" means "finite". The other useful variant is to replace $\Delta_{+}$by the category $\mid$Cat $_{f p} \mid$ of finitely presented categories. Then $J_{c a t}$ must be replaced by the Gabriel theory consisting of all finite colimit cocones so that $\operatorname{Mod}\left(J_{c a t}\right.$, Set) consists of the finite-limit-preserving (= left exact) functors from $\left|\mathbf{C a t}_{\mathrm{fp}}\right|^{\text {op }}$ to Set. Again all the results remain true.

(3.22) For any category $\mathbb{Q}$ and $J_{c a t}$ as in (3.16) we write cat $(\mathbb{Q})$ for the category of models of $J_{\text {cat }}$ in $\mathbb{Q}$; that is, $\operatorname{cat}(\mathbb{Q})=\operatorname{Mod}\left(J_{\text {cat }}, \mathbb{Q}\right)$. An object $A$ of $\operatorname{cat}(\mathbb{Q})$ is called a category in $Q$ (or a category object in $Q$, or an internal category of $Q$ ); it is precisely a simplicial object

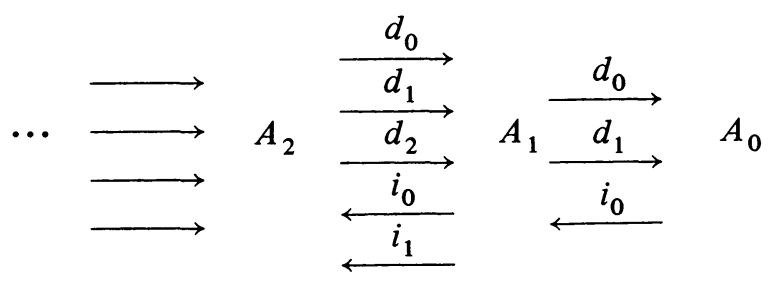

such that, for $n \geqslant 2$, the arrows $d_{0}, d_{n}: A_{n} \rightarrow A_{n-1}$ are the pullback of $d_{0}, d_{n-1}$ : $A_{n-1} \rightarrow A_{n-2}$. An arrow $f: A \rightarrow B$ in cat $(\mathcal{Q})$ is called a functor in $\mathcal{Q}$ and consists of arrows $f_{n}: A_{n} \rightarrow B_{n}, n \geqslant 0$, which constitute an arrow of simplicial objects.

(3.23) For all natural numbers $r$, we define $\operatorname{cat}^{r}(\mathbb{Q})$ inductively by $\operatorname{cat}^{0}(\mathbb{Q})=\mathscr{Q}$, cat $^{r+1}(\mathbb{Q})=\operatorname{cat}\left(\right.$ cat $\left.^{r}(\mathbb{Q})\right)$. The objects of cat $^{r}(\mathbb{Q})$ are called $r$-tple categories in $\mathbb{Q}$. Theorem (3.11) (recall, (3.21)) has the following corollary.

(3.24) TheOREM. Suppose $\mathbb{Q}$ is a small (respectively, finitely) complete, cartesian closed, locally small category. Then:

(a) $\operatorname{cat}^{r}(\mathbb{Q})$ is small (respectively, finitely) complete, cartesian closed and locally small; 
(b) if $F, P \in\left[\left(\Delta_{+}^{r}\right)^{\mathrm{op}}, \mathbb{Q}\right]$ and $F \in \operatorname{cat}^{r}(\mathbb{Q})$ then $[P, F] \in$ cat $^{r}(\mathbb{Q})$;

(c) if $\mathscr{Q}$ is locally presentable then so is cat ${ }^{r}(\mathfrak{Q})$ and the inclusion of cat $^{r}(\mathbb{Q})$ in $\left[\left(\Delta_{+}^{r}\right)^{\text {op }}, \mathbb{Q}\right]$ has a finite-product-preserving left adjoint.

(3.25) It follows also from the work of Freyd-Kelly [13], (3.24)(b) and (3.10) that, if $\mathscr{Q}$ is the category of compactly generated topological spaces, then the inclusion $\operatorname{cat}^{r}(\mathbb{Q}) \rightarrow\left[\left(\Delta_{+}^{r}\right)^{\text {op }}, \mathbb{Q}\right]$ has a finite-product-preserving left adjoint. There are many other such non-locally-presentable examples.

4. Fibrations between internal categories.

(4.1) For any locally small category $\mathcal{Q}$, we have a Yoneda embedding $\mathcal{Y}$ : $\mathbb{Q} \rightarrow\left[\mathfrak{Q}^{\text {op }}\right.$, Set $]$ which leads to a pullback diagram:

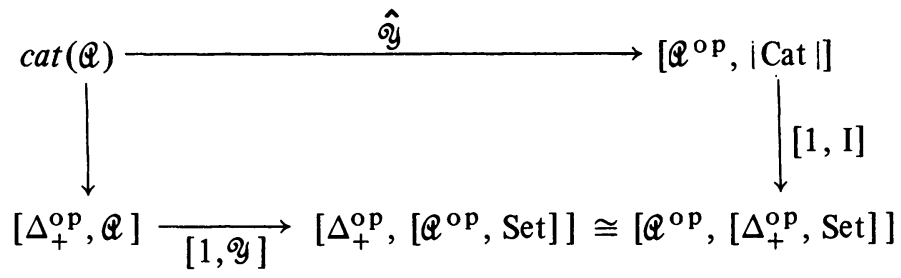

The functor $\hat{\mathcal{Y}}$ is fully faithful. For $f: A \rightarrow B$ in $\operatorname{cat}(\mathbb{Q})$, write $\mathscr{Q}(-, f): \mathcal{Q}(-, A) \rightarrow$ $\mathcal{Q}(-, B)$ for $\mathscr{Y} f$; so, for each $X \in \mathbb{Q}$, we have a functor $\mathcal{Q}(X, f): \mathcal{Q}(X, A) \rightarrow$ $\mathcal{Q}(X, B)$.

(4.2) For functors $f, g: A \rightarrow B$ in $\mathscr{Q}$, a transformation $\sigma: f \rightarrow g$ in $\mathbb{Q}$ is an arrow $\sigma$ : $A_{0} \rightarrow B_{1}$ in $\mathbb{Q}$ such that, for each object $X$ of $\mathscr{Q}$, the arrows $\mathbb{Q}(X, \sigma) a$ of $\mathscr{Q}(X, B)$ for $a \in \mathbb{Q}\left(X, A_{0}\right)$ are the components of a natural transformation from $\mathbb{Q}(X, f)$ to $\mathscr{Q}(X, g)$. It follows that $\sigma$ induces a modification $\mathscr{Q}(-, \sigma): \mathbb{Q}(-, f) \rightarrow \mathfrak{Q}(-, g)$.

(4.3) Write $\operatorname{Cat}(\mathcal{Q})$ for the 2-category of categories, functors and transformations in $\mathbb{Q}$, where the compositions are such that the assignment $\sigma \mapsto \mathbb{Q}(-, \sigma)$ enriches the functor $\hat{\mathcal{Y}}$ above (4.1) to a fully faithful 2-functor

$$
\hat{\mathcal{Y}}: \operatorname{Cat}(\mathfrak{Q}) \rightarrow\left[\mathscr{Q}^{\mathrm{op}}, \mathrm{Cat}\right] \text {. }
$$

(4.4) If $\mathscr{Q}$ is a finitely (small) complete category then $\operatorname{Cat}(\mathbb{Q})$ is a finitely (small) complete 2-category and the indexed limits are preserved by $\hat{\mathcal{Y}}$. Limits in [ $\left.\mathfrak{Q}^{\text {op }}, \mathrm{Cat}\right]$ are formed pointwise; in particular, $(2 \pitchfork F) X=[2, F X]$ for all $F: \mathbb{Q}^{\text {op }} \rightarrow$ Cat.

(4.5) If $\mathfrak{Q}$ is a finitely (small) complete, cartesian closed category then Cat $(\mathbb{Q})$ is a finitely (small) complete, cartesian closed 2-category. From (3.24) we have that $\operatorname{cat}(\mathscr{Q})=|\operatorname{Cat}(\mathscr{Q})|$ is cartesian closed and the internal hom is preserved by $\mathcal{Y}$. By (4.4), for any finite category $K$ we have $K \pitchfork[B, C] \simeq[B, K \pitchfork C]$ in cat $(\mathscr{Q})$ since it becomes true after applying $\mathcal{Y}$. This gives the (natural in $K$ ) isomorphisms

$$
\begin{aligned}
|\operatorname{Cat}|(K, \operatorname{Cat}(\mathscr{Q})(A,[B, C])) & \simeq \operatorname{cat}(\mathbb{Q})(A, K \pitchfork[B, C]) \\
& \simeq \operatorname{cat}(\mathbb{Q})(A,[B, K \pitchfork C]) \\
& \simeq \operatorname{cat}(\mathbb{Q})(A \times B, K \pitchfork C) \\
& \simeq|\operatorname{Cat}|(K, \operatorname{Cat}(\mathbb{Q})(A \times B, C))
\end{aligned}
$$


which are 2-natural in $A$. In particular, we have this for $K \in \Delta_{+}$which is dense in |Cat|. This gives:

$$
\operatorname{Cat}(\mathbb{Q})(A,[B, C]) \cong \operatorname{Cat}(\mathscr{Q})(A \times B, C)
$$

2-naturally in $A$. This proves (4.5).

(4.6) For any finitely complete category $\mathcal{Q}$ and categories $A, B$ in $\mathcal{Q}$, there is a monad $\Re_{B}^{A}$ on the category $Q \downarrow B_{0} \times A_{0}$ described as follows. For an object $\left(\begin{array}{c}q \\ p\end{array}\right)$ : $M \rightarrow B_{0} \times A_{0}$ over $B_{0} \times A_{0}$, the object $\mathscr{N}(M)$ over $B_{0} \times A_{0}$ is the limit of the diagram

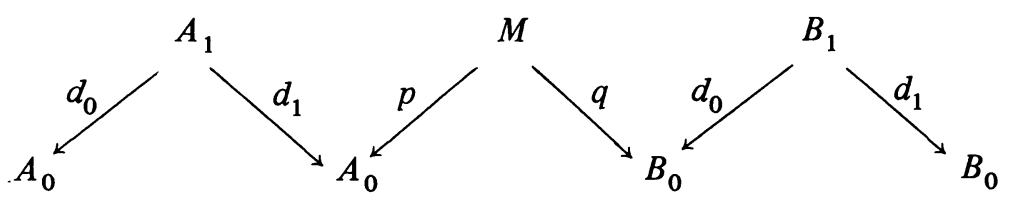

with arrow into $B_{0} \times A_{0}$ induced by the projections from $\mathscr{N}(M)$ to the outside $A_{0}, B_{0}$. The functoriality of limit gives that of $\mathscr{N}$. The components of the unit are induced by $i_{0}: A_{0} \rightarrow A_{1}, i_{0}: B_{0} \rightarrow B_{1}$ and the components of the multiplication by $d_{1}: A_{2} \rightarrow A_{1}, d_{1}: B_{2} \rightarrow B_{1}$ (3.22).

(4.7) Write $\operatorname{Prof}(B, A ; \mathcal{Q})$ (or simply $\operatorname{Prof}(B, A)$ ) for the category of EilenbergMoore algebras for the monad $\mathcal{N}$ on $\mathcal{Q} \downarrow B_{0} \times A_{0}$. The objects of $\operatorname{Prof}(B, A)$ are called profunctors from $B$ to $A$ in $\mathbb{Q}$.

(4.8) Suppose $P$ is a profunctor from $B$ to $A$ in $Q$. The structure on $P$ includes arrows $p: P \rightarrow A_{0}, q: P \rightarrow B_{0}$ and an action $c: \Re P \rightarrow P$. There are pullbacks:

$$
\begin{array}{ccccccccc}
(A \downarrow p)_{0} & \stackrel{d_{1}}{\rightarrow} & P & (q \downarrow B)_{0} & \rightarrow & B_{1} & \Re P & \rightarrow & (q \downarrow B)_{0} \\
\downarrow & & \downarrow p & d_{0} \downarrow & & \downarrow d_{0} & \downarrow & & \downarrow d_{0} \\
A_{1} & \overrightarrow{d_{1}} & A_{0} & P & \vec{q} & B_{0} & (A \downarrow p)_{0} & \overrightarrow{d_{1}} & P
\end{array}
$$

The right inverses $i_{0}, i_{0}$ for $d_{1}: A_{1} \rightarrow A_{0}, d_{0}: B_{1} \rightarrow B_{0}$ induce right inverses for $\Re P \rightarrow(A \downarrow p)_{0}, \Re P \rightarrow(q \downarrow B)_{0}$ which compose with the action $c$ to yield actions $c_{r}:(A \downarrow P)_{0} \rightarrow P, c_{l}:(q \downarrow B)_{0} \rightarrow P$ for the monads $(A \downarrow-)_{0},(-\downarrow B)_{0}$ on $P \in \mathbb{Q} \downarrow B_{0}$ $\times A_{0}$. (In fact, to give an action $c$ is precisely to give two actions $c_{r}, c_{l}$ satisfying the obvious "bimodule" condition.)

(4.9) Suppose $P$ is a category in $\operatorname{Prof}(B, A ; \mathcal{Q})$. So we have profunctors $P_{n}$ from $B$ to $A$ (3.22) and structure arrows $p, q, c$ for each $n>0$. We shall now describe a category $\Gamma P$ in $\mathbb{Q}$ which has $(\Gamma P)_{0}=P_{0}$. The object $(\Gamma P)_{1}$ of $\mathscr{Q}$ is the limit in the category $Q \downarrow \downarrow B_{0} \times A_{0}$ of the diagram

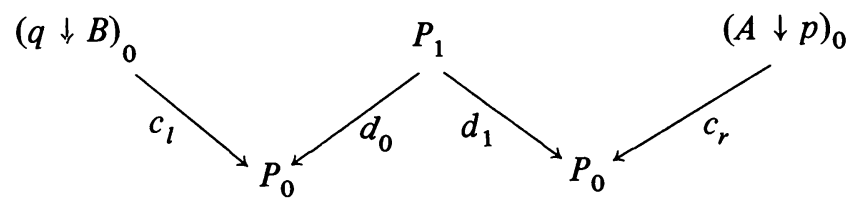

We obtain a graph $\Gamma P$ in $Q$ by taking $d_{0}, d_{1}:(\Gamma P)_{1} \rightarrow(\Gamma P)_{0}$ to be the projections from the limit to the left and right $\boldsymbol{P}_{0}$, respectively. It remains to describe a natural 
category structure on each of the graphs $\mathscr{Q}(K, \Gamma P)$ for $K \in \mathbb{Q}$. Suppose $x, x^{\prime}$ : $K \rightarrow P_{0}$ are objects of the graph $\mathcal{Q}(K, \Gamma P)$. An arrow from $x$ to $x^{\prime}$ in $\mathcal{Q}(K, \Gamma P)$ amounts (by the universal property of $\left.(\Gamma P)_{1}\right)$ to a triple $(\alpha, \xi, \beta)$ where $\alpha, \xi, \beta$ are arrows of $\mathbb{Q}(K, B), \mathbb{Q}(K, P), \mathbb{Q}(K, A)$ such that $d_{0} \beta=q x, d_{1} \alpha=p x^{\prime}, d_{0} \xi=$ $c_{l}(x, \beta), d_{1} \xi=c_{r}\left(\alpha, x^{\prime}\right)$. Suppose $(\beta, \xi, \alpha): x \rightarrow x^{\prime},\left(\beta^{\prime}, \xi^{\prime}, \alpha^{\prime}\right): x^{\prime} \rightarrow x^{\prime \prime}$ are arrows in $\mathcal{Q}(K, \Gamma P)$. "Associativity" and "bimodularity" give the equations

$$
\begin{gathered}
c_{l}\left(x, \beta^{\prime} \beta\right)=c_{l}\left(c_{l}(x, \beta), \beta^{\prime}\right), \quad c_{r}\left(\alpha, c_{r}\left(\alpha^{\prime}, x^{\prime \prime}\right)\right)=c_{r}\left(\alpha^{\prime} \alpha, x^{\prime \prime}\right), \\
c_{l}\left(c_{r}\left(\alpha, x^{\prime}\right), \beta^{\prime}\right)=c_{r}\left(\alpha, c_{l}\left(x^{\prime}, \beta^{\prime}\right)\right) .
\end{gathered}
$$

So $\xi: c_{l}(x, \beta) \rightarrow c_{r}\left(\alpha, x^{\prime}\right), \xi^{\prime}: c_{l}\left(x^{\prime}, \beta^{\prime}\right) \rightarrow c_{r}\left(\alpha^{\prime}, x^{\prime \prime}\right)$ in $\mathbb{Q}(K, P)$ induce $c_{l}\left(\xi, \beta^{\prime}\right)$ : $c_{l}\left(x, \beta^{\prime} \beta\right) \rightarrow c_{l}\left(c_{r}\left(\alpha, x^{\prime}\right), \beta^{\prime}\right), c_{r}\left(\alpha, \xi^{\prime}\right): c_{l}\left(c_{r}\left(\alpha, x^{\prime}\right), \beta^{\prime}\right) \rightarrow c_{r}\left(\alpha^{\prime} \alpha, x^{\prime \prime}\right)$. Composition for $\mathbb{Q}(K, \Gamma P)$ is defined by:

$$
\left(\beta^{\prime}, \xi^{\prime}, \alpha^{\prime}\right)(\beta, \xi, \alpha)=\left(\beta^{\prime} \beta, c_{r}\left(\alpha, \xi^{\prime}\right) c_{l}\left(\xi, \beta^{\prime}\right), \alpha^{\prime} \alpha\right) .
$$

(Compare with (1.4).)

(4.10) For each category $P$ in $\operatorname{Prof}(B, A ; \mathbb{Q})$, the category $\Gamma P$ in $\mathcal{Q}$ becomes an object of $\operatorname{Cat}(\mathscr{Q}) \downarrow B \times A$ by means of the functor $\Gamma P \rightarrow B \times A$ determined by the composites

$$
(\Gamma P)_{1} \rightarrow(q \downarrow B)_{0} \rightarrow B_{1},(\Gamma P)_{1} \rightarrow(A \downarrow p)_{0} \rightarrow A_{1} .
$$

(4.11) In fact, $\Gamma P$ supports a canonical structure of split fibration from $B$ to $A$ in $\operatorname{Cat}(\mathscr{Q})$ (2.3). The action $c: \mathcal{T}(\Gamma P) \rightarrow \Gamma P$ of $\mathcal{T}$ on $\Gamma P$ is induced by the action of $\Re$ on $P_{1}$ (of course, $c_{0}$ is precisely the action of $\Re$ on $P_{0}$ ).

(4.12) The functoriality of finite limits allows us to extend the assignment $P \mapsto \Gamma P$ to a 2 -functor

$$
\Gamma: \operatorname{Cat}(\operatorname{Prof}(B, A ; \mathscr{Q})) \rightarrow \operatorname{Spl}(B, A ; \operatorname{Cat}(\mathscr{Q})) .
$$

(4.13) THEOREM. The 2-functor $\Gamma$ is an equivalence.

(Since all constructions involved are finite limits, the proof reduces to the case $\mathbb{Q}=$ Set.)

(4.14) Consequently, $\Gamma$ induces an equivalence of categories:

$$
\operatorname{Prof}(B, A ; \mathcal{Q}) \simeq \operatorname{DSpl}(B, A ; \operatorname{Cat}(\mathcal{Q})) \text {. }
$$

(4.15) It is rather trivial to observe:

$$
\operatorname{Prof}(B, A ; \mathbb{Q})=\operatorname{Prof}\left(A^{\mathrm{op}} \times B, 1 ; \mathbb{Q}\right) \text {. }
$$

From this and (4.13) we deduce the rather less trivial equivalence of 2-categories

$$
\operatorname{Spl}(B, A ; \operatorname{Cat}(\mathcal{Q})) \simeq \operatorname{Spl}\left(A^{\text {op }} \times B, 1 ; \operatorname{Cat}(\mathcal{Q})\right) .
$$

A rather more precise statement than the last sentence of (1.14) is: for a finitely complete 2-category $\mathscr{K}$ with involution ( $)^{\text {op }}: \mathscr{K}^{\mathrm{co}} \rightarrow \mathscr{K}$ there need be no equivalence (4.16) with $\operatorname{Cat}(\mathscr{Q})$ replaced by $\mathscr{K}$. Since the pseudo-functoriality of $\operatorname{Spl}(\sim,-)(2.7)$ is given representably, it follows from (1.19) that the equivalences (4.16) are pseudo-natural in $A, B$.

(4.17) These considerations suggest a closer look at the categories $\operatorname{Prof}(B, 1 ; \mathbb{Q})$. On the one hand, regarding a profunctor $P$ from $B$ to 1 as a discrete category in $\operatorname{Prof}(B, 1 ; \mathcal{Q})$ we obtain a category $\Gamma P$ over $B(4.9)$. Then $(\Gamma P)_{1} \simeq(q \downarrow B)_{0}$, so 
there is a pullback

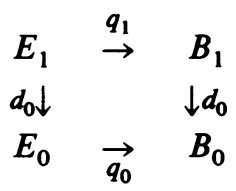

where $E=\Gamma P$. It is a classical (and easy) observation that $\Gamma$ induces an equivalence between $\operatorname{Prof}(B, 1 ; \mathcal{Q})$ and the full subcategory of $\operatorname{Cat}(\mathcal{Q}) \downarrow B$ consisting of those functors $q: E \rightarrow B$ in $\mathbb{Q}$ for which the above square is a pullback.

(4.18) On the other hand, for our profunctor $P$ from $B$ to 1, we can apply (2.9) with $E=\Gamma P, A=1$, and $A^{\prime}=1, B^{\prime}=Y$ discrete categories in $\mathcal{Q}$. This yields a functor $E(-, \sim)$ which we denote by:

$$
P_{Y}: \mathbb{Q}(Y, B) \rightarrow \mathbb{Q} \downarrow Y .
$$

More explicitly, for an object $b: Y \rightarrow B_{0}$ of $\mathcal{Q}(Y, B)$, the object $P_{Y} b$ over $Y$ is obtained by pulling back $q: P \rightarrow B_{0}$ along $b$; for an arrow $\beta: Y \rightarrow B_{1}$ of $\mathcal{Q}(Y, B)$ from $b$ to $b^{\prime}$, the arrows $P_{Y} b \rightarrow Y \stackrel{\beta}{\rightarrow} B_{1}, P_{Y} b \rightarrow P$ induce an arrow $P_{Y} b \rightarrow(q \downarrow B)_{0}$ which composes with the action $(q \downarrow B)_{0} \rightarrow P$ to induce an arrow $P_{Y} \beta: P_{Y} b \rightarrow$ $P_{Y} b^{\prime}$ over $Y$. Furthermore, the functors $P_{Y}$ are pseudo-natural in $Y$ where $Q \downarrow \downarrow Y$ is regarded as pseudo-functorial in $Y$ via pullback. Can we recapture $P$ from the pseudo-natural transformation $\mathbb{Q}(\sim, B) \rightarrow \mathbb{Q} \downarrow \sim$ ? Yes, up to isomorphism. This will follow from our analysis below of pseudo-natural transformations with domain $\mathcal{Q}(-, B)=\hat{\mathcal{Y}}_{B}$ where $B$ is a category in $\mathcal{Q}$ (see (5.18)).

\section{An extension of Yoneda's lemma.}

(5.1) Suppose $R: \mathcal{E} \rightarrow \mathbb{Q}$ is a functor. An arrow $u: E^{\prime} \rightarrow E$ in $\mathcal{E}$ is said to be left cartesian (with respect to $R$ ) when, for all $v: E^{\prime \prime} \rightarrow E$ in $\mathcal{E}$ and $t: R E^{\prime \prime} \rightarrow R E^{\prime}$ in $Q$ such that $R v=R u \cdot t$, there exists a unique $w: E^{\prime \prime} \rightarrow E^{\prime}$ in $\mathcal{E}$ such that $v=u w$ and $R w=t$. In this section we deal only with "left cartesian" so we shall abbreviate this to "cartesian". It is easily seen that, if $u: E^{\prime} \rightarrow E$ is cartesian, an arrow $u^{\prime}: E^{\prime \prime} \rightarrow E^{\prime}$ is cartesian precisely when $u u^{\prime}$ is cartesian.

(5.2) Suppose the following square in $\mathcal{E}$ is taken by $R$ to a pullback in $Q$, and that $u$ is cartesian.

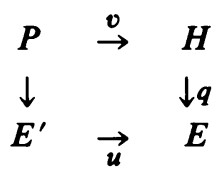

The square is a pullback in $\mathcal{E}$ if and only if $v$ is cartesian.

(5.3) The functor $R: \mathcal{E} \rightarrow \mathbb{Q}$ is called a left fibration (or $\mathcal{E}$ is a fibration from 1 to $\mathcal{Q}$, or $\mathcal{E}$ is a left fibred category over $\mathcal{Q}$ ) when, for each object $E$ of $\mathcal{E}$ and each arrow $r: A \rightarrow R E$ in $Q$, there exists a cartesian arrow $\chi_{r}: r^{*} E \rightarrow E$ such that $R \chi_{r}=r$. It follows from (5.2) in this case, if $\mathcal{Q}$ has pullbacks then cartesian arrows have pullbacks with arbitrary arrows in $\mathcal{E}$.

(5.4) To relate the notion of fibration with that of split fibration (1.10), suppose $F: A^{\mathrm{op}} \rightarrow$ Cat is a functor. The Grothendieck construction (1.15) gives a category $\mathcal{G}_{1}^{A}(F)$ over $A$. An arrow $(\alpha, \xi):(a, x) \rightarrow\left(a^{\prime}, x^{\prime}\right)$ (see (1.4)) is cartesian precisely 
when $\xi$ is an isomorphism. If $\xi$ is an identity we say $(\alpha, \xi)$ is split cartesian. It is easy to see that $\mathcal{G}_{1}^{A}(F)$ is a fibration from 1 to $A$; indeed, split cartesian arrows can be found to fulfil the condition.

(5.5) A left fibration $R: \mathcal{E} \rightarrow \mathbb{Q}$ determines a pseudo-functor $\mathcal{E}: \mathbb{Q}^{\text {op }} \rightarrow$ CAT as follows. For each object $A$ of $\mathcal{Q}, \mathcal{E} A$ is defined by the pullback:

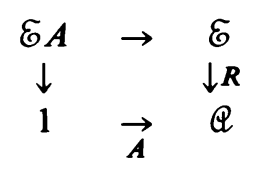

it is the fibre of $\mathcal{E}$ over $A$. For $r: A \rightarrow B$, the functor $\mathcal{E} r: \mathcal{E} B \rightarrow \mathcal{E} A$ is given by $(\mathcal{E} r) E=r^{*} E(5.3)$ and $\chi_{r} \cdot \delta h=h \chi_{.}$. The isomorphisms $\mathcal{E}(s r) \simeq \mathcal{E} r \cdot \mathcal{E} s, \mathcal{E} 1_{A} \simeq$ $1_{\delta A}$ are induced by the universal property of cartesian arrows.

(5.6) For categories $\mathcal{E}, \mathcal{F}$ over $\mathcal{Q}$, write $\operatorname{Cart}^{\mathbb{Q}}(\mathscr{F}, \mathcal{E})$ for the category of cartesian-arrow-preserving functors from $\mathscr{F}$ to $\mathcal{E}$ over $\mathcal{Q}$ and natural transformations over $\mathbb{Q}$.

(5.7) It is a classical result of Grothendieck that, for left fibred categories $\mathcal{E}, \mathcal{F}$ over $\mathcal{Q}$, the category $\operatorname{Cart}^{\mathbb{Q}}(\mathcal{F}, \mathcal{E})$ is isomorphic to the category of pseudo-natural transformations from $\mathcal{F}$ to $\mathcal{E}$ (as pseudo-functors (5.5)) and modifications. Indeed, every pseudo-functor $\mathbb{Q}^{\text {op }} \rightarrow$ CAT is isomorphic to one arising from a left fibration (5.5), so we have an equivalence between the 2-category of pseudo-functors from $\mathbb{Q}^{\text {op }}$ to CAT and a sub-2-category of CAT $\downarrow \mathbb{Q}$. The equivalence is a simple extension of the Grothendieck construction $\mathcal{G}_{1}^{\mathbb{R}}$ of $\$ 1$.

(5.8) Suppose $B$ is a category in $Q$. Let $Q \cdot \downarrow \cdot B$ denote the Grothendieck constructon $\mathcal{G}_{1}^{\mathcal{Q}}$ evaluated at the functor $\hat{\text { Oิ }} B=\mathscr{Q}(-, B): \mathbb{Q}^{\text {op }} \rightarrow$ Cat (4.3). Explicitly, using (1.4), an object of $\mathcal{Q} \cdot \downarrow \cdot B$ is an arrow $b: X \rightarrow B_{0}$ in $\mathcal{Q}$, and an arrow $(f, \beta): b \rightarrow b^{\prime}$ consists of arrows $f: X \rightarrow X^{\prime}, \beta: X \rightarrow B_{1}$ in $\mathcal{Q}$ such that $d_{0} \beta=b$, $d_{1} \beta=b^{\prime} f$.

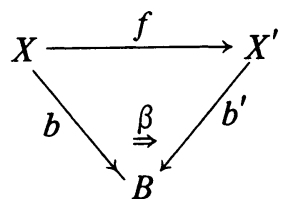

Note that $(f, \beta)$ is split cartesian precisely when $\beta$ is an identity transformation $b \rightarrow b^{\prime} f$ in $\operatorname{Cat}(\mathbb{Q})$; that is, when $b=b^{\prime} f$ and $\beta=i_{0} b$.

(5.10) There is a functor $(Q \cdot \downarrow \cdot B)^{\mathrm{op}} \rightarrow$ Cat described as follows. The value at $b$ is the comma category $b \downarrow \mathbb{Q}(X, B)$. The value at $(f, \beta)$ is the functor $b^{\prime} \downarrow \mathbb{Q}\left(X^{\prime}, B\right)$ $\rightarrow b \downarrow \mathbb{Q}(X, B)$ given by "pasting on" the diagram (5.9) in the 2-category $\operatorname{Cat}(\mathscr{Q})$.

(5.11) The following diagrams represent arrows in $Q \cdot \downarrow \cdot B$.
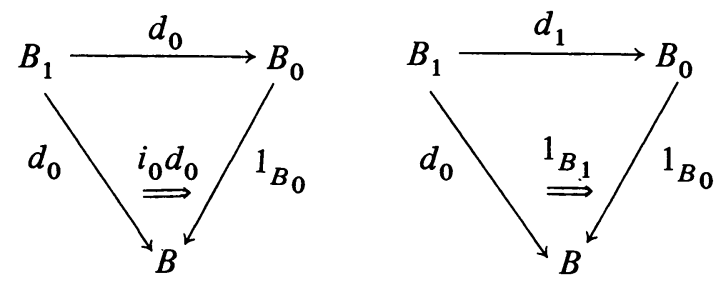
Let $B$ denote the graph in $Q \cdot \downarrow \cdot B$ with these arrows as domain and codomain arrows, respectively. Then $\hat{B}$ projects into $\mathbb{Q}$ as the underlying graph of $B$.

(5.12) Proposition. For each category $B$ in $Q$ there is a structure of category on the graph $\hat{B}(5.11)$ in $Q \cdot \downarrow \cdot B$ with the following properties:

(i) the functor of $(5.10)$ is isomorphic to the functor $(\mathbb{Q} \cdot \downarrow \cdot B)(-, \hat{B}):(\mathbb{Q} \cdot \downarrow \cdot B)^{\text {op }}$ $\rightarrow$ Cat;

(ii) the projection $\mathbb{Q} \cdot \downarrow \cdot B \rightarrow \mathbb{Q}$ takes $\hat{B}$ to $B$ as simplicial objects;

(iii) each $d_{0}: \hat{B}_{n+1} \rightarrow \hat{B}_{n}$ is split left cartesian.

Proof. An arrow $b \rightarrow \hat{B}_{0}$ amounts precisely to an object of $b \downarrow \mathbb{Q}(X, B)$, and an arrow $b \rightarrow \hat{B}_{1}$ amounts precisely to an arrow of $b \downarrow \mathbb{Q}(X, B)$. It easily follows that the graph $\hat{B}$ represents the composite of the functor (5.10) with the underlying functor from Cat to graphs. The desired objects $\hat{B}_{n}$ can all be defined by the appropriate pullbacks (3.22) which all exist since $d_{0}: \hat{B}_{1} \rightarrow \hat{B}_{0}$ is split cartesian (5.2). By Yoneda's lemma the simplicial structure on $\hat{B}$ can be induced from the pointwise category structure on the functor of (5.10). So (i) holds. Yoneda's lemma gives (ii), and (iii) is clear from the construction.

(5.13) Suppose $R: \mathcal{E} \rightarrow \mathbb{Q}$ is a functor (respectively, a split left fibration) and $B$ is a category in $\mathcal{Q}$. Write $\mathcal{E}_{p} B$ (respectively, $\mathcal{E} B$ ) for the full subcategory of $\operatorname{Cat}(\mathcal{E}$ ) consisting of those categories $E$ in $\mathcal{E}$ which are taken as simplicial objects to $B$ by $R$ and which have $d_{0}: E_{1} \rightarrow E_{0}$ cartesian (respectively, split cartesian). If $B$ is discrete then $\mathcal{E}_{p} B$ (respectively, $\mathcal{E}_{B}$ ) is equivalent (respectively, isomorphic) to the fibre of $\mathcal{E}$ over $B(5.5)$. For any $B$ in the split case, $\mathcal{E}_{p} B$ is equivalent to $\mathscr{E} B$.

(5.14) Evaluation at $\hat{B}(5.12)$ provides a functor

$$
\operatorname{eval}_{\hat{B}}: \operatorname{Cart}^{\mathscr{Q}}(\mathscr{Q} \cdot \downarrow \cdot B, \mathcal{E}) \rightarrow \mathcal{E}_{p} B
$$

For a cartesian-arrow-preserving functor $N: \mathbb{Q} \cdot \downarrow \cdot B \rightarrow \mathcal{E}$ over $A$, it follows from (5.2), (5.12) that the value of $N$ at the simplicial object $\hat{B}$ gives an object $N \hat{B}=e v a l_{\hat{B}} N$ of $\mathcal{E}_{p} B$. A natural transformation $\theta: N \rightarrow N^{\prime}$ over $\mathbb{Q}$ provides, for each $n$, an arrow $\theta_{\hat{B}_{n}}: N \hat{B}_{n} \rightarrow N^{\prime} \hat{B}_{n}$ in $\mathcal{E} B_{n}$ which, by naturality, form a functor $\theta_{\hat{B}}=\operatorname{eval}_{\hat{B}} \theta: N \hat{B} \rightarrow N^{\prime} \hat{B}$.

(5.15) Theorem. Suppose $R: \mathcal{E} \rightarrow \mathbb{Q}$ is a functor and $B$ is a category in $\mathbb{Q}$.

(i) The functor eval $\hat{B}_{\hat{B}}$ of (5.14) is fully faithful.

(ii) If $R$ is a left fibration then eval $\hat{B}_{\hat{B}}$ is an equivalence of categories.

(iii) If $R$ is a split left fibration then eval $_{B}$ restricts to an isomorphism of categories:

$$
\operatorname{Spl}(\mathscr{Q}, 1)(\mathscr{Q} \cdot \downarrow \cdot B, \mathcal{E}) \simeq \mathcal{E} B
$$

(iv) For all functors $F: \mathbb{Q}^{\text {op }} \rightarrow$ Cat there is an isomorphism of categories

$$
\left[\mathcal{Q}^{\text {op }}, \text { Cat }\right](\mathcal{Q}(-, B), F) \cong \mathcal{G}_{1}^{\mathbb{Q}}(F) B \text {. }
$$

Proof. (i) Take $N, N^{\prime}$ objects of $\operatorname{Cart}^{\mathbb{Q}}(\mathbb{Q} \cdot \downarrow \cdot B, \mathcal{E})$ and suppose $\phi: N \hat{B} \rightarrow N^{\prime} \hat{B}$ is a functor in $\mathcal{E}$. For each object $b$ of $Q \cdot \downarrow \cdot B$, the commutative square 


$$
\begin{array}{rll}
N b & \stackrel{\theta_{b}}{\rightarrow} & N^{\prime} b \\
N\left(b, i_{0} b\right) \downarrow & & \downarrow N^{\prime}\left(b, i_{0} b\right) \\
N \hat{B}_{0} & \overrightarrow{\phi_{0}} & N^{\prime} \hat{B}_{0}
\end{array}
$$

defines $\theta_{b}: N b \rightarrow N^{\prime} b$ in $\delta X$ since the vertical arrows are cartesian. With the data (5.9), the above square, the equations $\left(b, i_{0} b\right)=\left(d_{0}, i_{0} b\right)\left(\beta, i_{0} b\right), d_{0} \phi_{1}=\phi_{0} d_{0}$, and the fact that $d_{0}=N^{\prime}\left(d_{0}, i_{0} b\right)$ is cartesian, we deduce the equation $\phi_{1} N\left(\beta, i_{0} b\right)=$ $N^{\prime}\left(\beta, i_{0} b\right) \theta_{b}$. This equation together with $\left(d_{1}, 1_{B_{1}}\right)\left(\beta, i_{0} b\right)=\left(b^{\prime}, i_{0} b^{\prime}\right)(f, \beta), d_{1} \phi_{1}=$ $\phi_{0} d_{1}$, and the fact that $N^{\prime}\left(b^{\prime}, i_{0} b^{\prime}\right)$ is cartesian, imply $N^{\prime}(f, \beta) \theta_{b}=\theta_{b^{\prime}}(f, \beta)$. So we have a natural transformation $\theta: N \rightarrow N^{\prime}$ over $Q$. The assignment $\phi \mapsto \theta$ is clearly inverse to $\theta \mapsto \theta \hat{B}$. This proves (i).

(ii) Suppose $R$ is a left fibration and $E$ is an object of $\mathcal{E}_{p} B$. Define $N$ : $\mathcal{Q} \cdot \downarrow \cdot B \rightarrow \mathcal{E}$ as follows. Put $N b=b^{*} E_{0} \in \mathcal{E} X$ (5.3). Take $(f, \beta): b \rightarrow b^{\prime}$ as in (5.9). Since $d_{0}: E_{1} \rightarrow E_{0}$ is cartesian, so is

$$
\beta^{*} E_{1} \stackrel{x_{\beta}}{\rightarrow} E \stackrel{d_{0}}{\rightarrow} E_{0} .
$$

But $d_{0} \beta=b$, so there is an isomorphism $\beta^{*} E_{1} \simeq b^{*} E_{0}$ which commutes with the cartesian arrows into $E_{0}$. Define $N(f, \beta)$ by the condition $R N(f, \beta)=f$ and the commutative diagram

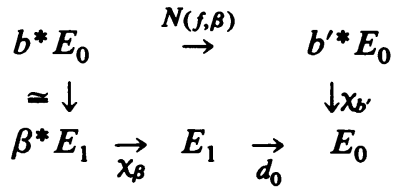

To show that $N$ preserves composition take $(f, \beta),\left(f^{\prime}, \beta^{\prime}\right)$ with composite $\left(f^{\prime} f, \gamma\right)$ where $\gamma=\beta^{\prime} f \cdot \beta: b \Rightarrow b^{\prime \prime} f^{\prime} f$ in $\operatorname{Cat}(\mathcal{Q})$. Let $\xi, \xi^{\prime}: b^{*} E_{0} \rightarrow E_{1}$ denote the left side and top of the commutative diagram

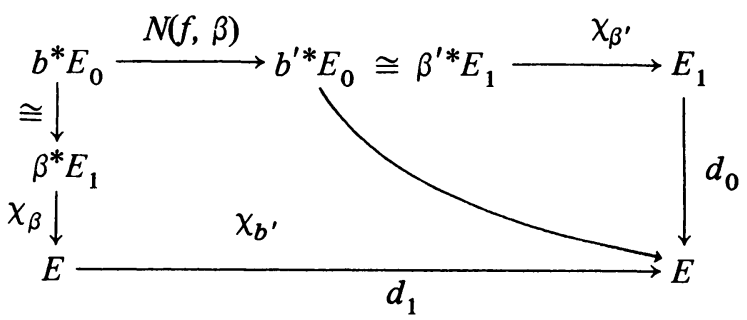

The diagram shows that $\xi, \xi^{\prime}$ are a composable pair of arrows in the category $\mathcal{E}\left(b^{*} E_{0}, E\right)$. Let $\zeta: b^{*} E_{0} \rightarrow E_{1}$ denote their composite. Since $R$ takes $E$ to $B$, $R \zeta=\gamma$. Now $d_{0} \zeta=d_{0} \xi$ and $d_{0}, \xi$ are cartesian, so $\zeta$ is cartesian. So $\zeta$ is the composite

$$
b^{*} E_{0} \approx \gamma^{*} E_{1} \stackrel{x_{\beta}}{\rightarrow} E_{1} .
$$

Also $d_{1} \zeta=d_{1} \xi^{\prime}$, so $\chi_{\beta^{\prime \prime}} N\left(f^{\prime}, \beta^{\prime}\right) N(f, \beta)=\chi_{\beta^{\prime \prime}} N\left(f^{\prime} f, \gamma\right)$ from which the cartesian $\chi_{\beta^{\prime \prime}}$ can be cancelled. So $N$ preserves composition. 
Since $d_{0}: E_{1} \rightarrow E_{0}$ is cartesian, we deduce that, for any $b: X \rightarrow B_{0}$, the arrow $\chi_{i_{0} b}$ is the composite

$$
\left(i_{0} b\right)^{*} E_{1} \cong b^{*} E_{0} \stackrel{x_{b}}{\rightarrow} E_{0} \stackrel{i_{0}}{\rightarrow} E_{1} .
$$

So, if $b=b^{\prime} f$, then $\chi_{b^{\prime}} N\left(f, i_{0} b\right)$ is cartesian. So $N\left(f, i_{0} b\right)$ is cartesian. It follows that $N$ preserves identities and cartesian arrows.

To see that $N \hat{B} \cong E$ it follows from Proposition (5.12) that it suffices to see this at the level of underlying graphs. From the definition of $N$ on arrows and (5.11) it is immediate that $N$ takes $d_{0}, d_{1}: \hat{B}_{1} \rightarrow \hat{B}_{0}$ to an isomorph of $d_{0}, d_{1}: E_{1} \rightarrow E_{0}$. This proves (ii).

(iii) In this case the isomorphisms such as $b^{*} E_{0} \simeq \beta^{*} E_{1}$ can be chosen to be identities in a coherent way in (ii). So we obtain $N \hat{B}=E$ when $N$ preserves split cartesian arrows.

(iv) The isomorphism of (iii) with $\mathscr{E}=\mathcal{G}_{1}^{\mathbb{Q}}(F)$ composes with the effect on hom-categories of the Grothendieck construction (1.9) to yield the result.

(5.16) Observe that when $B$ and $F$ are discrete, Theorem (5.15)(iv) reduces to the usual Yoneda lemma (Mac Lane [21, p. 61]).

(5.17) We now return to the remarks at the end of (4.18). In Theorem (5.15), take $\mathcal{E}=[2, \mathbb{Q}]$ and $R=\left[\partial_{1}, 1_{\mathbb{Q}}\right]$, the codomain functor. An arrow in $[2, \mathbb{Q}]$ is cartesian precisely when it is a pullback square in $\mathcal{Q}$. Assuming $\mathbb{Q}$ has pullbacks, we see that $R$ is a left fibration. Combining (4.17), (5.13) and (5.15)(ii), we obtain the equivalence:

$$
\operatorname{Cart}^{\mathbb{Q}}(\mathbb{Q} \cdot \downarrow \cdot B,[2, \mathbb{Q}]) \simeq \operatorname{Prof}(B, 1 ; \mathbb{Q}) .
$$

The left-hand side of this is isomorphic (5.7) to the category of pseudo-functors and pseudo-natural transformations from $Q(\sim, B)$ to $Q \downarrow \downarrow \sim$. It follows that a profunctor $P$ from $B$ to 1 in $Q$ amounts up to isomorphism to a pseudo-natural transformation $\mathbb{Q}(\sim, B) \rightarrow \mathbb{Q} \downarrow \sim$.

6. Internal full subcategories. For a category $B$ in a finitely complete category $Q$, the external to $\mathbb{Q}$ notion of a pseudo-natural transformation $N: \mathbb{Q}(-, B) \rightarrow \mathbb{Q} \downarrow-$ internalizes via the extended Yoneda lemma to the notion of a profunctor $P$ from $B$ to 1 (4.18), (5.18). Properties of $N$ translate to properties of $P$; for example, we can ask what it means in terms of $P$ for the functors $N_{Y}$ to be full, faithful, left exact, right adjoints, etc., for all $Y$. This section is concerned with the fully faithful requirement.

(6.1) An internal full subcategory $(S, I)$ of $\mathbb{Q}$ consists of a category $S$ in $\mathbb{Q}$ together with a profunctor $I$ from $S$ to 1 such that, for all objects $Y$ of $\mathbb{Q}$, the functor

$$
I_{Y}: \mathbb{Q}(Y, S) \rightarrow \mathbb{Q} \downarrow Y
$$

(as described in (4.18)) is fully faithful. Each $(S, I)$ gives (4.17) an object $\Gamma I \rightarrow S$ of $[2, \operatorname{Cat}(\mathcal{Q})]$, and so we obtain a 2-category of internal full subcategories of $\mathcal{Q}$. 
(6.2) TheOREM. For an arrow $q: I \rightarrow S_{0}$ in a finitely complete category $\mathcal{Q}$, the following structures are equivalent.

(i) An internal full subcategory $(S, I)$ such that the object of $Q \downarrow S_{0}$ underlying the profunctor $I$ is $q$;

(ii) a graph $d_{0}, d_{1}: S_{1} \rightarrow S_{0}$ in $\mathbb{Q}$ and an arrow $s: I_{S_{1}}\left(d_{0}\right) \rightarrow I_{S_{1}}\left(d_{1}\right)$ in $\mathbb{Q} \downarrow S_{1}$ such that the components

$$
\begin{array}{ccc}
\mathcal{Q}\left(Y, S_{1}\right) & \stackrel{\mathcal{Q}\left(Y, d_{0}\right)}{\rightarrow} & \mathbb{Q}\left(Y, S_{0}\right) \\
\mathcal{Q}\left(Y, d_{0}\right) \downarrow & \stackrel{\sigma Y}{\Rightarrow} & \downarrow I_{Y} \\
\mathbb{Q}\left(Y, S_{0}\right) & \overrightarrow{I_{Y}} & \mathbb{Q} \downarrow Y
\end{array}
$$

of the modification $\sigma$ corresponding to $s$ induce isomorphisms of $\operatorname{sets} \mathcal{Q}\left(Y, S_{1}\right) \simeq$ $I_{Y} \downarrow I_{Y}$;

(iii) a cartesian internal hom

$$
\left(\begin{array}{l}
d_{0} \\
d_{1}
\end{array}\right): S_{1} \rightarrow S_{0} \times S_{0}
$$

for the objects $q \times 1: I \times S_{0} \rightarrow S_{0} \times S_{0}, 1 \times q: S_{0} \times I \rightarrow S_{0} \times S_{0}$ in the category $\mathbb{\downarrow} \downarrow S_{0} \times S_{0}$.

Proof. (i) $\Rightarrow$ (ii) To say $I_{Y}: \mathbb{Q}(Y, S) \rightarrow \mathbb{Q} \downarrow Y$ is fully faithful is to say that the induced functor $[2, \mathbb{Q}(Y, S)] \rightarrow I_{Y} \downarrow I_{Y}$ is an isomorphism of categories. Restricting this isomorphism to objects gives the isomorphism of sets $Q\left(Y, S_{1}\right) \simeq I_{Y} \downarrow I_{Y}$ as required for (ii).

(ii) $\Leftrightarrow$ (iii) Take $x, y: Y \rightarrow S_{0}$ and let $z: I_{Y}(x) \rightarrow S_{0} \times S_{0}$ be the product of $\left(\begin{array}{l}x \\ y\end{array}\right)$ : $Y \rightarrow S_{0} \times S_{0}$ and $q \times 1: S_{0} \rightarrow S_{0} \times S_{0}$ in $Q \downarrow S_{0} \times S_{0}$. Then we obtain bijections:
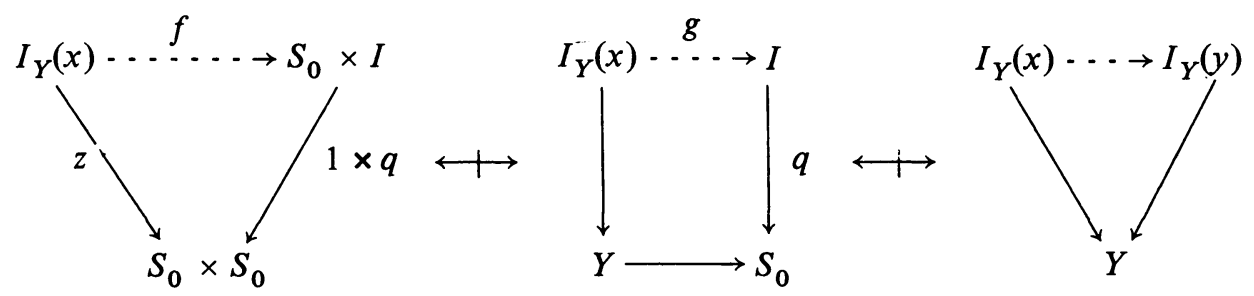

To say $\left(\begin{array}{l}d_{0} \\ d_{1}\end{array}\right)$ is a cartesian internal hom as in (iii) is to say that arrows $f$ are in natural bijection with arrows $\xi: Y \rightarrow S_{1}$ such that $d_{0} \xi=x, d_{1} \xi=y$. On the other hand, elements of $I_{Y} \downarrow I_{Y}$ as in (ii) are precisely triples $(x, g, y)$ as above. The equivalence of (ii) and (iii) follows easily.

(ii) $\Rightarrow$ (i) The graph $d_{0}, d_{1}: I_{Y} \downarrow I_{Y} \rightarrow \mathbb{Q}\left(Y, S_{0}\right)$ enriches to a category by defining composition by $(y, h, z)(x, g, y)=(x, h g, z)$. Assuming (ii) this structure transfers by Yoneda's lemma to yield a category $S$ in $\mathcal{Q}$ with underlying graph $d_{0}, d_{1}$ : $S_{1} \rightarrow S_{0}$ and such that $\mathcal{Q}(Y, S)$ is equivalent to the full subcategory of $\mathbb{Q} \downarrow Y$ consisting of the objects $I_{Y}(x)$ over $Y$. 
(6.3) It follows from (6.2) that any two internal full subcategories of $\mathcal{Q}$ with the same arrow $q: I \rightarrow S_{0}$ underlying their profunctors must be isomorphic. Furthermore, if $Q \downarrow S_{0} \times S_{0}$ is cartesian closed, then any arrow $q: I \rightarrow S_{0}$ can be enriched to an internal full subcategory $(S, I)$ of $\mathscr{Q}$.

(6.4) Theorem. Suppose $(S, I)$ is an internal full subcategory of a finitely complete category $\mathbb{Q}$. For each category $B$ in $Q$, the functor

$$
(\Gamma I)(1, \sim): \operatorname{Cat}(\mathscr{Q})(B, S) \rightarrow \operatorname{DSpl}(B, 1 ; \operatorname{Cat}(\mathbb{Q}))
$$

(see (4.17), (2.9)) is fully faithful.

Proof. Recall (2.9) that $\operatorname{DSpl}(B, 1 ; \operatorname{Cat}(\mathbb{Q}))$ is a full subcategory of $\operatorname{Cat}(\mathbb{Q}) \downarrow B$. So it must be shown that $(\Gamma I)(1, \sim)$ is fully faithful when regarded as landing in $\operatorname{Cat}(\mathbb{Q}) \downarrow B$. Take functors $h, k: B \rightarrow S$ in $\mathbb{Q}$ and suppose we have an arrow

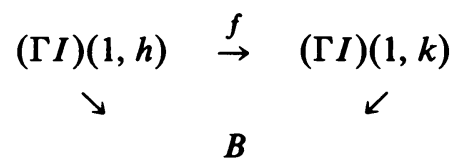

in $\operatorname{Cat}(\mathscr{Q}) \downarrow B$. By Theorem 6.2(ii) the arrows $h_{0}, k_{0}: B_{0} \rightarrow S_{0}$ and $f: I_{B_{0}}(h) \rightarrow I_{B_{0}}(k)$ determine an arrow $\xi: B_{0} \rightarrow S_{0}$ with $d_{0} \xi=h_{0}, d_{1} \xi=k_{0}$. It is not hard to see that $\xi$ : $h \rightarrow k$ is a transformation (4.2) in $Q$, indeed, the unique one with $(\Gamma I)(1, \xi)=f$.

(6.5) TheOREM. Suppose $(S, I)$ is an internal full subcategory of a finitely complete, cartesian closed category $\mathcal{Q}$. The following data determine a fibrational cosmos (2.11):

(a) the 2-category Cat $(\mathbb{Q})$ (see (4.5));

(b) the 2-functor $\mathscr{P}=\left[(-)^{\mathrm{op}}, S\right]$ with left adjoint $\mathscr{P} *=[-, S]^{\mathrm{op}}$;

(c) for each object $A$ of $\operatorname{Cat}(\mathbb{Q})$, the discrete fibration $\in_{A}$ from $\mathcal{P} A$ to $A$ corresponding under (4.16) to the discrete fibration $\bar{\epsilon}_{A}$ from $A^{\mathrm{op}} \times \mathscr{P} A$ to 1 obtained by pullback (recall (4.17)):

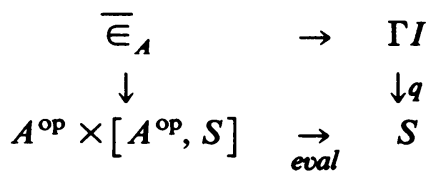

Proof. By (4.5), $\mathcal{K}=\operatorname{Cat}(\mathcal{Q})$ is finitely complete and cartesian closed. So:

$$
\begin{aligned}
\mathcal{K}^{\mathrm{coop}}\left([A, S]^{\mathrm{op}}, B\right) & =\mathscr{K}\left(B,[A, S]^{\mathrm{op}}\right)^{\mathrm{op}} \\
& =\mathcal{K}\left(B^{\mathrm{op}},[A, S]\right) \cong \mathscr{K}\left(A,\left[B^{\mathrm{op}}, S\right]\right) .
\end{aligned}
$$

This gives $\mathscr{P} * \dashv \mathscr{P}$. The composite

$$
\mathcal{K}(B, \mathscr{P} A) \cong \mathcal{K}\left(A^{\text {op }} \times B, S\right) \stackrel{I(1, \sim)}{\rightarrow} \operatorname{DSpl}\left(A^{\text {op }} \times B, 1 ; \mathcal{K}\right) \underset{(4.16)}{\simeq} \operatorname{DSpl}(B, A ; \mathcal{K})
$$

is fully faithful (6.4) and pseudo-natural in $A, B$ (2.7) (4.16). The image of the identity of $\mathscr{P} A$ under this composite when $B=\mathscr{P} A$ is precisely $\epsilon_{A}$ as in (c). The argument of Yoneda's lemma can mimicked to show that the composite must be $\in_{A}(A, \sim)$. So we have (2.11)(i), (ii). 
(6.6) For functors $a: K \rightarrow A, a^{\prime}: L \rightarrow A$ in $\mathcal{Q}$, the image under (4.16) of the discrete fibration $a \downarrow a^{\prime}$ from $L$ to $K$ (see (2.4), (2.13)) is the discrete fibration $a \bar{\downarrow} a^{\prime}$ from $K^{\text {op }} \times L$ to 1 called the twisted comma category of $a, a^{\prime}$. For any object $X$ of $Q$, an arrow $X \rightarrow a \bar{\downarrow} a^{\prime}$ amounts to a triple $(u, \alpha, v)$ where $u: X \rightarrow K, v: X \rightarrow L$ and $\alpha: a u \rightarrow a^{\prime} v$ in $\operatorname{Cat}(\mathbb{Q})$; a transformation between arrows corresponding to $(u, \alpha, v),(r, \beta, s)$ consists of transformations $\sigma: r \rightarrow u, \tau: v \rightarrow s$ such that the following commutes.

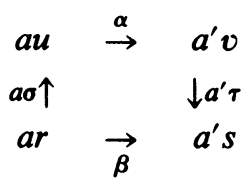

In particular, $A \bar{\downarrow} A$ is called the twisted arrow category of $A$.

(6.7) A functor $a: K \rightarrow A$ in $Q$ is admissible (2.13) in the fibrational cosmos of (6.5) if and only if there exists a functor $\bar{A}(a, 1): K^{\mathrm{op}} \times A \rightarrow S$ for which there is a pullback

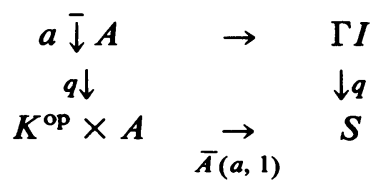

In particular, an admissible category $A$ in $Q$ has a hom-functor $A^{\text {op }} \times A \rightarrow S$ pullback along which takes $\Gamma I$ to the twisted arrow category of $A$. This provides the details for Street-Walters [30, p. 376].

(6.8) In the situation of (6.4), if $j: T \rightarrow S$ is a fully faithful functor in $\mathcal{Q}$ (that is, $A \downarrow A \cong j \downarrow j)$ and $J$ is the pullback of $I$ along $j_{0}$ then $(T, J)$ is an internal full subcategory of $Q$.

7. Gabriel theories and internal full subcategories.

(7.1) Any internal full subcategory $(S, I)$ of Set yields a fully faithful functor

$$
S=\operatorname{Set}(1, S) \stackrel{I_{1}}{\rightarrow} \text { Set } \downarrow 1=\text { Set. }
$$

On the other hand, suppose $\iota: T \rightarrow$ Set is a fully faithful functor where $T$ is a small category. Let $S$ be the full subcategory of Set consisting of the sets $\iota t, t \in T$. Then $\iota$ induces an equivalence $T \simeq S$ and the inclusion of $S$ in Set gives fully faithful functors

$$
\operatorname{Set}(Y, S)=[Y, S] \rightarrow[Y, \text { Set }] \simeq \operatorname{Set} \downarrow Y
$$

which are pseudo-natural in $Y$. It follows (5.18), (6.1) that $S$ bears a structure of internal full subcategory, called the canonical one. From this we see that every internal full subcategory of Set is equivalent to a small full subcategory of Set with its canonical internal full subcategory structure. We shall now do a similar analysis for [ ${ }^{\text {op }}$, Set] in place of Set.

(7.2) For each functor $F$ : $e^{\text {op }} \rightarrow$ Set there is a functor

$$
\Lambda_{F}:\left[\mathcal{C}^{\mathrm{op}}, \text { Set }\right] \downarrow F \rightarrow\left[\mathcal{G}_{1}^{\mathcal{C}}(F)^{\mathrm{op}}, \text { Set }\right]
$$


defined as follows (refer to (1.4)). For $\theta: G \rightarrow F,\left(\Lambda_{F} \theta\right)(U, s)=\{x \in G U \mid(\theta U) x=$ $s\}$ for $s \in F U$, and $\left(\Lambda_{F} \theta\right) h$ is the restriction of $G h$. For $\gamma: \theta \rightarrow \theta^{\prime},\left(\Lambda_{F} \gamma\right)_{\left(U_{s}\right)} x=$ $\gamma_{U} x$.

(7.3) Proposition. The functors $\Lambda_{F}$ of (7.2) are the components of a pseudo-natural equivalence

$$
\Lambda \sim:[\text { eop, Set }] \downarrow \sim \simeq\left[\mathcal{G}_{1}^{e}(\sim)^{\text {op }}, \text { Set }\right]
$$

between pseudo-functors from [ $\left[e^{o p}, \text { Set }\right]^{\text {op }}$ to CAT.

(Note that the codomain of $\Lambda$ is actually a functor.)

Proof. Using the fact that a functor into $\mathcal{G}(F)$ is a discrete left fibration if and only if it is an arrow of discrete left fibrations over $\mathcal{C}$, we have the following equivalences which are pseudo-natural (1.19) in $F$ and whose composite is $\Lambda_{F}$ :

$$
\begin{aligned}
{\left[\mathcal{C}^{\text {op }}, \text { Set }\right] \downarrow F } & \underset{(1.12)}{\simeq} D \operatorname{Spl}(1, \mathcal{C}) \downarrow \mathcal{G}(F) \\
& \simeq D \operatorname{Spl}(1, \mathcal{G}(F)) \underset{(1.12)}{\simeq}\left[\mathcal{G}(F)^{\text {op }}, \text { Set }\right]
\end{aligned}
$$

(7.4) In particular, (7.3) applies when $F=\mathcal{C}(-, U)=\mathcal{Y} U$ (4.1) to yield a pseudo-natural equivalence

$$
\left[\mathcal{C}^{\text {op }} \text {, Set }\right] \downarrow \mathscr{Y} \sim \simeq\left[(\mathcal{\downarrow} \sim)^{\text {op }} \text {, Set }\right]
$$

whose component at $U$ is

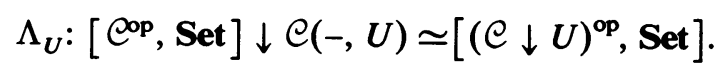

(7.5) Theorem. Suppose $\mathcal{C}$ is a small category, $T: \mathcal{C}^{\circ} \rightarrow$ Cat is a pseudo-functor, and

$$
\text { : } T \rightarrow\left[(\mathcal{\downarrow} \sim)^{\text {op }}, \text { Set }\right]
$$

is a pseudo-natural transformation with fully faithful components. There exist an internal full subcategory $(S, I)$ of [ [ ${ }^{\text {op }}$, Set] and a pseudo-natural equivalence $T \simeq S$ which are unique up to isomorphism with the property that $S$ is a full subfunctor of $\left[(\mathcal{\sim} \sim)^{\text {op }}\right.$, Set $]$ satisfying:

(i) $\iota$ is the composite of the equivalence $T \simeq S$ with the inclusion $S \rightarrow$ $\left[(\mathcal{\downarrow} \sim)^{\mathrm{op}}\right.$, Set];

(ii) the inclusion mentioned in (i) is isomorphic to the composite

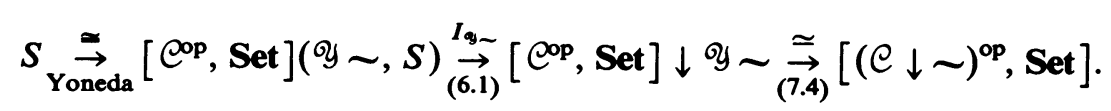

Proof. Let $R$ denote the small full subcategory of Set consisting of all the sets $((\iota U) t) h$ where $h: V \rightarrow U$ in $\mathcal{C}$ and $t \in T U$. Then $\iota$ factors through the full subfunctor $\left[(\mathcal{\sim} \sim)^{\text {op }}, R\right]$ ]: $\mathcal{C}^{\text {op }} \rightarrow$ Cat of $\left[(\mathcal{\downarrow} \sim)^{\text {op }}\right.$, Set]: epp $^{\text {op }} \rightarrow$ CAT. For $U \in \mathcal{C}$, let $S U$ denote the full subcategory of $\left[(\mathcal{U})^{\mathrm{op}}, R\right]$ consisting of those functors which are isomorphic to functors of the form $(\iota U) t$. If $f: U^{\prime} \rightarrow U$ in $\mathcal{C}$ and $\phi \in S U$

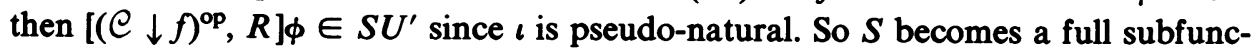
tor of $\left[(\bigcup \downarrow)^{\text {op }}, R\right]$ and $\iota$ induces a pseudo-natural equivalence $T \simeq S$ as required for (i). 
The inclusion $S \rightarrow\left[\left(\downarrow_{\downarrow} \sim\right)^{\text {op }}\right.$, Set] induces fully faithful functors

$$
\left[\bigodot^{\text {op }}, \operatorname{Set}\right](F, S) \rightarrow\left[\bigodot^{\text {op }}, \operatorname{SET}\right]\left(F,\left[(\bigodot \downarrow \sim)^{\text {op }} \text {, Set }\right]\right)
$$

which are natural in $F$. Using (1.2) with $X$ replaced by Set ${ }^{\text {op }}$, we see that the codomain of the above displayed functor is naturally isomorphic to [ $\mathcal{G}(F)^{\text {op }}$, Set]. Combining this with (7.3) we obtain fully faithful functors

$$
[\text { [op, Set }](F, S) \rightarrow\left[\text { Cop }^{\text {op }} \text { Set }\right] \downarrow F
$$

which are pseudo-natural in $F$. So, by (5.18), there is an internal full subcategory $(S, I)$ of [ [ $\bigodot^{o p}$, Set] for which $I_{F}(4.18)$ is the above displayed fully faithful functor. This gives (ii).

Uniqueness is left to the reader.

(7.6) For a full subfunctor $S$ of $\left[\left(\bigcup_{\downarrow} \sim\right)^{\text {op }}\right.$, Set] which lands in Cat we obtain an internal full subcategory $(S, I)$ of [eop, Set] satisfying (7.5)(ii); this is called the canonical internal full subcategory structure on $S$. It follows from (7.5) that every internal full subcategory of [ [ ${ }^{\circ}$, Set] is equivalent (6.1) to a canonical one. We call $\left[\left(\downarrow_{\downarrow} \sim\right)^{\text {op }}\right.$, Set]: $e^{\text {op }} \rightarrow$ CAT the gross internal full subcategory; it would be an internal full subcategory if it were not so big. Any full subfunctor of the gross internal full subcategory which lands in Cat is an internal full subcategory of [ $\bigodot^{\text {op }}$, Set], and all such arise in this way up to equivalence.

(7.7) One particular case of (7.5) when $C$ has pullbacks arises from the Yoneda embeddings

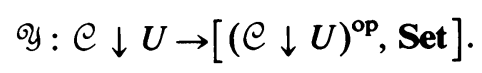

Regarding the domain $C \downarrow U$ as pseudo-functorial in $U$ via pullback and the codomain as functorial in $U$ as usual (1.1), we see that these embeddings are the components of a pseudo-natural transformation. Applying (7.5) yields an internal full subcategory $(C, I)$ of $\left[e^{\text {op }}\right.$, Set] called the realization of $\mathcal{C}_{\text {in }}\left[\bigodot^{\text {op }}\right.$, Set]. Note

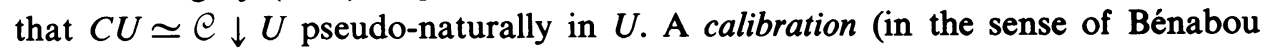
[3]) of the category $C$ is precisely a full sub-internal-full-subcategory of $(C, I)$ which is suitably "cocomplete".

(7.8) Another particular case arises from the small full subcategory 2 of Set which becomes an internal full subcategory $(2,1)$ of Set with $q=\partial_{1}: 1 \rightarrow 2=2_{0}$. The canonical internal full subcategory (7.6) of [ ${ }^{\text {op }}$, Set] corresponding to the subfunctor $\left[\left(\bigcup_{\downarrow}\right)^{\text {op }}, 2\right]$ of $\left[\left(C_{\downarrow} \sim\right)^{\text {op }}\right.$, Set] has the form $(\Omega, 1)$ where $q U: 1 \rightarrow$ $(\Omega U)_{0}=\left[(\bigodot \downarrow U)^{\mathrm{op}}, 2\right]_{0}$ picks out the composite $(\bigodot \downarrow U)^{\mathrm{op}} \rightarrow \mathbf{1} \underset{\mathrm{a}_{1}}{\rightarrow}$. A crible (or sieve) on a category $\mathcal{E}$ (Giraud [16]) is a set $\mathscr{R}$ of objects such that, for all $f$ : $A \rightarrow B$, if $B \in \Re$ then $A \in \Re$. We can identify a crible $\Re$ on $\mathcal{E}$ with the functor $\mathcal{E}^{\mathrm{op}} \rightarrow 2$ which takes the objects in $R$ to 1 and the other objects to 0 . So $\Omega U$ is the

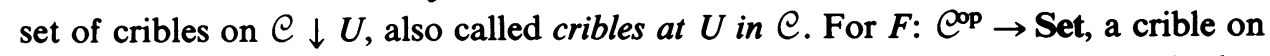
$\mathcal{G}(F)(1.4)$ is easily seen to amount to a subfunctor of $F$. It follows from (1.2) that $(\Omega, 1)$ is the subobject classifier (see Johnstone [18]) for [ ${ }^{\circ \text { op }}$, Set]; that is, every monomorphism $G \leadsto F$ in [ [ep, Set] is isomorphic to an object in the image of the fully faithful functor 


$$
\left[\bigodot^{\text {op }}, \text { Set }\right](F, \Omega) \rightarrow\left[\bigodot^{\infty}, \text { Set }\right] \downarrow F
$$

determined by $(\Omega, 1)$. We shall see below that full sub-internal-full-subcategories of $(\Omega, 1)$ which are suitably "cocomplete" correspond to Grothendieck topologies on e.

(7.9) We now recall (Gabriel-Ulmer [15], Freyd-Kelly [13]) the translation of the "model" condition (3.6) into the "orthogonality" condition. Recall that an arrow $f$ : $A \rightarrow B$ in a category $Q$ is said to be orthogonal to an object $C$ when the function $\mathscr{Q}(f, C): \mathbb{Q}(B, C) \rightarrow \mathbb{Q}(A, C)$ is an isomorphism. Suppose $\mathcal{C}$ is a small category and $U$ is an object of $\mathcal{C}$. Each cocone $\tau: D \Rightarrow U$ gives a cocone $\mathcal{Y}_{\tau}: \mathcal{Y}_{D} \Rightarrow \mathcal{Y} U$ in [ ${ }^{\text {op }}$, Set] which, provided the domain of $D$ is small, induces an arrow

$$
\bar{\tau}: \operatorname{col} \mathscr{Y} D \rightarrow \mathscr{Y} U
$$

from the colimit of the composite of $D$ with the Yoneda embedding. For a functor $F:$ eop $\rightarrow$ Set, to say $F \tau: F U \Rightarrow F D$ is a limit for $F D$ is precisely to say that $\bar{\tau}$ is orthogonal to $F$ in [£op, Set].

(7.10) On the other hand, any natural transformation $\alpha: R \rightarrow C_{(-, U)}$ corresponds to an arrow $\mathcal{G}(\alpha): \mathcal{G}(R) \rightarrow \mathcal{C} \downarrow U$ of discrete left fibrations (1.12). Composing this with the canonical natural transformation $\lambda$, we obtain a cocone $\lambda \mathcal{S}(\alpha)$ :

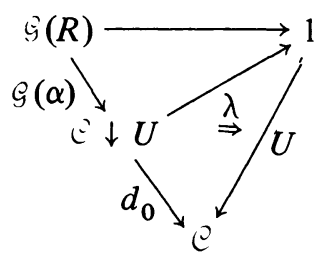

For any functor $F$ : $e^{o p} \rightarrow$ Set, we have the canonical isomorphism

$$
\left[e^{\text {op }}, \operatorname{Set}\right](R, F) \simeq \lim \left(\mathcal{G}(R)^{\text {op }} \rightarrow \operatorname{eop}^{\text {op }} \rightarrow \text { Set }\right) .
$$

It follows that $F$ is orthogonal to $\alpha$ if and only if $F$ takes $\lambda \mathcal{G}(\alpha)$ to a limit cone. Also, the colimit of $\mathcal{G}(R) \rightarrow \mathcal{C} \rightarrow$ 过 $\left[\mathcal{C}^{\text {op }}\right.$, Set] is isomorphic to $R$; so the arrow $\bar{\tau}$ obtained from $\tau=\lambda \mathcal{G}(\alpha)$ via (7.9) is isomorphic to $\alpha$.

(7.11) In the situation of (7.9) we can apply (7.10) to $\bar{\tau}$ : col $\mathcal{Y} D \rightarrow \mathcal{Y} U$. This gives a factorization of the original cocone $\tau$ as:

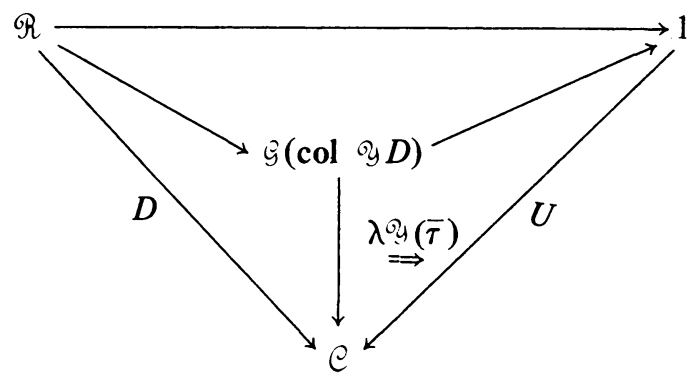


where the triangle on the right-hand side is none other than the "comprehensive factorizaton" of $D$ into a final functor and a discrete left fibration (Street-Walters [31]). The finality of $\Re \rightarrow \mathcal{G}(\operatorname{col} \mathcal{Y} D)$ means that we can replace $\tau$ by $\lambda \mathcal{G}(\bar{\tau})$ without affecting the models.

(7.12) Two Gabriel theories (3.5) on the same category are said to be equivalent when they have the same set-valued models.

(7.13) Proposition. Each Gabriel theory on a small category is equivalent to one for which the domain functors of all the cocones are discrete left fibrations.

(7.14) For a Gabriel theory $J$ on a small category $\mathcal{C}$, write $\bar{J}(U)$ for the small full subcategory of [ $\complement^{\text {op }}$, Set] $\downarrow \mathcal{C}(-, U)$ consisting of the objects $\bar{\tau}$ for $\tau \in J(U)$ (7.9). Up to equivalence, to give a Gabriel theory is to give, for each $U$, a small full subcategory $\bar{J}(U)$ of [ $\bigodot^{\text {op }}$, Set] $\downarrow \bigodot(-, U)$ (see (7.10)).

(7.15) A Gabriel theory $J$ on $C$ is said to be pullback stable when, for each $\alpha \in \bar{J}(U)$ and each $f: V \rightarrow U$ in $\mathcal{C}$, there exists $\beta \in \bar{J}(V)$ for which there is a pullback:

$$
\begin{array}{ccc}
R^{f} & \stackrel{\beta}{\rightarrow} & \mathcal{C}(-, V) \\
\downarrow & & \downarrow \mathcal{C}(-, f) \\
\boldsymbol{R} & \rightarrow & \mathcal{C}(-, U)
\end{array}
$$

in [ $\mathcal{C}^{\text {op }}$, Set]. In other words, $\bar{J}$ becomes a sub-pseudo-functor of [ [ op, Set] $\downarrow \mathcal{O} \sim$.

(7.16) Proposition. Suppose $J$ is a pullback stable Gabriel theory on a small category $\mathcal{C}$. If the square

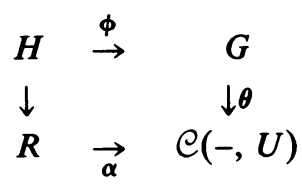

is a pullback in [@op, Set] and $\alpha \in \bar{J}(U)$ (7.13) then $\phi$ is orthogonal (7.8) to every model of $J$ in Set.

Proof. Since $G \cong \operatorname{col}_{(V, x)} \mathcal{C}(-, V)$ where $(V, x) \in \mathcal{G}(G)$ (see (7.10)), form the pullbacks

$$
\begin{array}{ccc}
\boldsymbol{H}_{\boldsymbol{x}} & \stackrel{\boldsymbol{\phi}_{x}}{\rightarrow} & \mathcal{C}(-, V) \\
\downarrow & & \downarrow \mathcal{C}\left(-, f_{x}\right) \\
\boldsymbol{R} & \vec{\alpha} & \mathcal{C}(-, U)
\end{array}
$$

for each $(V, x) \in \mathcal{G}(G)$, where $\theta$ is induced by the $f_{x}$. Since $J$ is pullback stable,

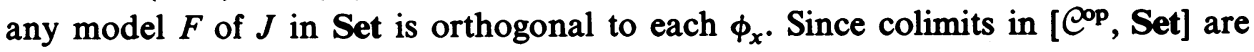
universal, $H$ is the colimit in [e colimits by the $\phi_{x}$, the function [ $\left.\bigodot^{\text {op }}, \operatorname{Set}\right](\phi, F)$ is induced on limits by the functions [ $\left.\bigodot^{\text {op }}, \operatorname{Set}\right]\left(\phi_{x}, F\right)$ which are all isomorphisms. So $\phi$ is orthogonal to $F$.

(7.17) Any internal full subcategory $(S, I)$ of [ $\mathcal{C}^{\text {op }}$, Set] determines a pullback stable Gabriel theory $J$ on $\mathcal{C}$ with $J(U)$ consisting of the cocones $\lambda \mathcal{G}(\alpha)$ (see (7.10)) 
where $\alpha$ is such that there is a pullback:

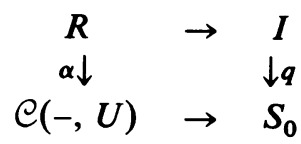

Recalling (6.7), we see that $\alpha \in \bar{J}(U)$ precisely when it is an admissible arrow between discrete objects of the 2-category Cat [ ${ }^{\text {op }}$, Set] $=$ [ ${ }^{\text {op }}$, Cat]. So we call $J$ the Gabriel theory on $\mathcal{C}$ admitted by $(S, I)$. Equivalent internal full subcategories admit equivalent Gabriel theories.

(7.18) On the other hand, a pullback stable Gabriel theory $J$ determines an internal full subcategory $(S, I)$ of [ ${ }^{\circ \text { op }}$, Set] by applying $(7.5)$ to the pseudo-natural transformation (see (7.15)):

$$
\bar{J} \rightarrow\left[\mathcal{C o p}^{\text {op }} \text { Set }\right] \downarrow \mathcal{Y} \sim \stackrel{(7.4)}{\simeq}\left[(\mathcal{\sim} \sim)^{\text {op }}, \text { Set }\right] .
$$

The Gabriel theory admitted by $(S, I)$ is then equivalent to $J$. However, equivalent pullback stable Gabriel theories can lead to wildly nonequivalent internal full subcategories. For example, a category of models for a pullback stable Gabriel theory on 1 must be equivalent to 1, 2 or Set, yet the set of equivalence classes of internal full subcategories of Set is not small.

(7.19) Suppose $Q: \mathcal{E} \rightarrow \mathcal{C}$ is a discrete right fibration and $E$ is an object of $\mathscr{E}$. Each cocone $\tau: D \Rightarrow Q E$ in $\mathcal{C}$ has a unique lifting to a cocone $\chi_{\tau}: \tau^{*}(E) \Rightarrow E$ in $\mathcal{E}$ (5.3). So a Gabriel theory $J$ on $\mathcal{C}$ lifts to a Gabriel theory $J_{\mathcal{E}}$ on $\mathcal{E}$ with $J_{\mathscr{E}}(E)=\left\{\chi_{r} \mid \tau \in J(Q E)\right\}$. Each element $\alpha$ of $\bar{J}(Q E)$ (7.14) determines an element $\alpha^{Q}$ of $\bar{J}_{\mathscr{E}}(E)$ such that there is a pullback

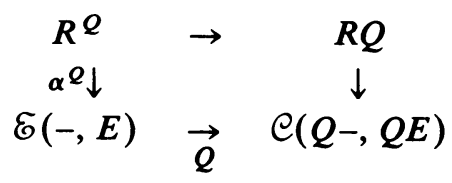

in $\left[\mathcal{E}^{\text {op }}\right.$, Set].

(7.20) Proposition. In the situation of (7.19), if $J$ is pullback stable then so is $J_{\mathscr{E}}$.

Proof. Take $E \in \mathcal{E}, \tau: D \Rightarrow Q E$ in $J(Q E)$, and $h: E^{\prime} \rightarrow E$ in $\mathcal{E}$. Since $J$ is pullback stable there exists a cocone $\tau^{\prime}: D^{\prime} \Rightarrow Q E^{\prime}$ in $J\left(Q E^{\prime}\right)$ such that $\bar{\tau}^{\prime}$ is a pullback of $\bar{\tau}$ along $\mathcal{C}(-, Q h)$. To this pullback apply $\left[Q^{\text {op }}, 1\right]:\left[\mathcal{C}^{\text {op }}\right.$, Set $] \rightarrow$ $\left[\mathcal{E}^{\text {op }}\right.$, Set] and pullback the result along $Q: \mathcal{E}(-, E) \rightarrow \mathcal{C}(Q-, Q E)$. This shows that $\bar{\chi}_{r^{\prime}}$ is a pullback of $\bar{\chi}_{r}$ along $\mathcal{E}(-, h)$. So $J_{\mathscr{E}}$ is pullback stable.

(7.21) Proposition. For any model F: $e^{\text {op }} \rightarrow$ Set of a Gabriel theory $J$ on $\mathcal{C}$, the equivalence $\Lambda$ (7.3) restricts to an equivalence

$$
\operatorname{Mod}(J, \text { Set }) \downarrow F \simeq \operatorname{Mod}\left(J_{\mathcal{G}(F)}, \text { Set }\right) .
$$

Proof. In the notation of (7.2) we must show that $G$ is a model for $J$ if and only if $\Lambda_{F} \theta$ is a model for $J_{\mathcal{G}(F)}$. 
Suppose $G$ is a model of $J$. Take $(U, s) \in \mathcal{G}(F), \tau: D \Rightarrow U$ in $J(U)$, and $x \in \lim \left(\Lambda_{F} \theta\right) \tau^{*}(U, s)$. Then $x_{i} \in\left(\Lambda_{F} \theta\right)\left(D i,\left(F \tau_{i}\right) s\right)$ and $\left(\Lambda_{F} \theta\right)(D \xi) x_{j}=x_{i}$ for all $\xi:$ $i \rightarrow j$ in the domain of $D$. So $\theta(D i) x_{i}=\left(F \tau_{i}\right) s$ and $(G D \xi) x_{j}=x_{i}$. So $x \in \lim G D$. So there exists a unique $y \in G U$ such that $\left(G \tau_{i}\right) y=x_{i}$. Since $F$ is a model of $J$, the calculation $\left(F \tau_{i}\right)(\theta U) y=(\theta D i)\left(G \tau_{i}\right) y=(\theta D i) x_{i}=\left(F \tau_{i}\right) s$ implies $(\theta U) y=s$. So $y$ $\in\left(\Lambda_{F} \theta\right)(U, s)$. So $\Lambda_{F} \theta$ is a model of $J_{G(F)}$.

Suppose $\Lambda_{F} \theta$ is a model of $J_{\mathcal{G}(F)}$. Take $U \in \mathcal{C}, \tau \in J(U)$ and $x \in \lim G D$. Then $x_{i} \in G D i$ and $(G D \xi) x_{j}=x_{i}$. So $(F D \xi)(\theta D j) x_{j}=(\theta D i)(G D \xi) x_{j}=(\theta D i) x_{i}$. So there is a unique $s \in F U$ such that $\left(F \tau_{i}\right) s=(\theta D i) x_{i}$. This gives $x \in \lim \left(\Lambda_{F} \theta\right) \tau^{*}(U, s)$. So there exists a unique $y \in\left(\Lambda_{F} \theta\right)(U, s)$ with $\left(\left(\Lambda_{F} \theta\right) \tau_{i}\right) y=x_{i}$. So $y \in G U$ with $\left(G \tau_{i}\right) y=x_{i}$. Two such $y$ would give the same $x, s$ and so be equal.

(7.22) A crible at $U$ in $C$ (7.8) can be identified with a full subcategory $R$ of $\circlearrowright \downarrow U$ for which the inclusion is a discrete left fibration, and this in turn can be identified with the cocone in $\mathcal{C}$ obtained by composing the inclusion $R \leftrightarrow \bigodot \downarrow U$ with the canonical $\lambda$ (7.10). A Grothendieck topology on a small category $\mathcal{C}$ is a Gabriel theory $J$ on $\mathcal{C}$ such that each $J(U)$ consists of cribles at $U$ and satisfies axioms T1), T2), T3) of SGA4 [1]. Condition T1) amounts precisely to the condition that $J$ be pullback stable. Since the domains of all the cocones in this case are discrete right fibrations, to give $J$ is to give $\bar{J}$. Moreover, $\bar{J}$ is a full subfunctor of $\Omega$ (7.8). It is not hard to see that conditions T2), T3) amount to saying that the inclusion $\bar{J} \rightarrow \Omega$ should be classified (7.8) by an arrow $j: \Omega \rightarrow \Omega$ satisfying $1<j$, $j j<j$. The monad $j$ on $\Omega$ in the 2-category [£op, Cat] (Street [25]) is the LawvereTierney topology on the topos [ $\bigodot^{\text {op }}$, Set] (see Johnstone [18]) corresponding to $J$ on e.

(7.23) A Grothendieck topos is a category which is equivalent to a category of Set-valued models for a Grothendieck topology on a small category. It has been shown by Gabriel-Ulmer [15] that, for a Gabriel theory $J$ on a small category $\mathcal{C}$ which is pullback stable and such that the elements $\alpha$ of each $\bar{J}(U)$ are monomorphisms, the category Mod( $J$, Set) is a Grothendieck topos. In other words, if $J$ is as in (7.22), except that T2), T3) are not necessarily valid, then there is a Grothendieck topology which is equivalent to $J$. One naturally asks the question: what are the categories of models for pullback stable Gabriel theories? This question is answered by Theorem (7.25) below.

(7.24) A category $\mathcal{Q}$ is called internally complete (or a "closed span category" by Day [7]) when, for each object $X$ of $\mathcal{Q}$, the category $\mathscr{Q} \downarrow X$ is cartesian closed. Spanier's quasi-topological spaces form an internally complete category which is not locally presentable.

(7.25) THEOREM. A category $\mathbb{Q}$ is locally presentable and internally complete if and only if there exists a pullback stable Gabriel theory $J$ on a small category such that $\operatorname{Mod}(J$, Set $) \simeq \mathbb{Q}$. Furthermore in this case, it is possible to find a $J$ for which the representables are models.

Proof. Suppose $J$ is a pullback stable Gabriel theory on a small category $\mathcal{C}$ and $\operatorname{Mod}(J$, Set $) \simeq \mathbb{Q}$. For all $V \in \mathcal{C}$ and $\alpha: R \rightarrow \mathcal{C}(-, U)$, we have the pullback 


$$
\begin{array}{ccc}
e(-, V) \times R & \stackrel{1 \times \alpha}{\rightarrow} & C(-, V) \times C(-, U) \\
p r_{2} \downarrow & & \downarrow p r_{2} \\
R & \vec{a} & C(-, U)
\end{array}
$$

By Proposition (7.16), if $\alpha \in \bar{J}(U)$ then every model $F$ of $J$ is orthogonal to the top arrow $1 \times \alpha$. It follows that the cartesian internal hom $[\mathcal{C}(-, V), F]$ in [ $\mathcal{C}^{\text {op }}$, Set] is orthogonal to $\alpha$. So $[\mathcal{C}(-, V), F]$ is a model for $J$ if $F$ is. Since every $P$ : $\mathcal{C}^{\circ p} \rightarrow$ Set is a colimit of representables it follows that $[P, F]$ is a model for $J$. By Theorem (3.11), $\operatorname{Mod}(J$, Set) is cartesian closed.

By Proposition (7.20), $J_{\mathcal{G}(F)}$ is pullback stable and so the above argument applies to yield $\operatorname{Mod}\left(J_{\mathcal{G}(F)}\right.$, Set) cartesian closed. This implies, by Proposition (7.21), that $\operatorname{Mod}(J$, Set), and hence $\mathcal{Q}$ is internally complete. That $\mathcal{Q}$ is locally presentable follows from (3.7).

Conversely, suppose $\mathcal{Q}$ is locally presentable and internally complete. By (3.7), $\mathbb{Q} \simeq \operatorname{Mod}(J$, Set) for some Gabriel theory $J$ on a small category $\mathcal{C}$. It is well known (see Gabriel-Ulmer [15], Bastiani-Ehresmann [2]) and easy (replace $C$ by the full subcategory of $\operatorname{Mod}(J$, Set) consisting of the reflections of the representables) that we may suppose that the representables $\mathcal{C}(-, U)$ are models for $J$. The objects $\mathcal{C}(-, V) \rightarrow \mathcal{C}(-, U)$ of $\operatorname{Mod}(J$, Set $) \downarrow \mathcal{C}(-, U)$ are strongly generating in [eop, Set] $\downarrow \mathcal{C}(-, U)$ so that Theorem (3.10) applies to yield that $\operatorname{Mod}(J$, Set) $\downarrow \mathcal{C}(-, U)$ is closed under exponentiation in [eop, Set] $\downarrow \mathcal{C}(-, U)$. Now suppose we have a pullback square

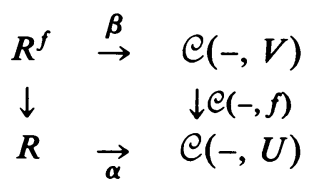

in [ $\mathcal{C}^{\text {op }}$, Set] and a model $F$ for $J$. Then the internal hom $H \rightarrow \mathcal{C}(-, U)$ of $\mathcal{C}(-, V) \rightarrow \mathcal{C}(-, U)$ and $F \times \mathcal{C}(-, U) \rightarrow \mathcal{C}(-, U)$ is such that $H$ is a model for $J$.

Suppose $\alpha \in \bar{J}(U)$. We shall prove $\beta$ is orthogonal to $F$. For this, take any $\theta$ : $R^{f} \rightarrow F$. This induces a unique $R^{f} \rightarrow F \times \mathcal{C}(-, U)$ over $\mathcal{C}(-, U)$. Since $R^{f}$ is the product of $R$ and $\mathcal{C}(-, V)$ over $\mathrm{C}(-, U)$, this corresponds to a unique $R \rightarrow H$ over $\mathcal{C}(-, U)$. Since $\alpha$ is orthogonal to $H$, this arrow factors uniquely through $\alpha$ to yield $\mathcal{C}(-, U) \rightarrow \boldsymbol{H}$. Since $\mathcal{C}(-, U)$ is a model, this arrow is a right inverse for $\boldsymbol{H} \rightarrow$ $\mathcal{C}(-, U)$. So $\theta: R^{f} \rightarrow F$ corresponds to an arrow over $\mathcal{C}(-, U)$ from the terminal object $\mathcal{C}(-, U)$ to $H$; that is, to an arrow from $\mathcal{C}(-, V)$ to $F \times \mathcal{C}(-, U)$ over $\mathcal{C}(-, U)$; that is, to an arrow $\phi: \mathcal{C}(-, V) \rightarrow F$. One readily traces through to find $\phi \beta=\theta$. So $\beta$ is orthogonal to $F$.

For each $f: V \rightarrow U$ in $C$ and $\alpha \in \bar{J}(U)$, choose a pullback $\beta$ of $\alpha$ along $C(-, f)$. Form a new Gabriel theory $J^{\prime}$ on $\mathcal{C}$ by adding to the cocones of $J$ those cocones arising as in (7.10) from the natural transformations $\beta$. By (7.10) and the above $J^{\prime}$ is equivalent to $J$. So $Q \simeq \operatorname{Mod}\left(J^{\prime}\right.$, Set $)$, the representables are models for $J^{\prime}$, and $J^{\prime}$ is pullback stable. 
(7.26) TheOREM. Suppose $J$ is a pullback stable Gabriel theory on a small category

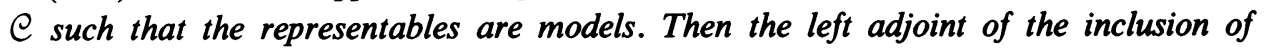
$\operatorname{Mod}(J$, Set) in [£op, Set] preserves pullbacks of those pairs of arrows whose codomains are subfunctors of models.

Proof. Let $L$ be the left adjoint referred to; then the left adjoint of the inclusion

$$
\operatorname{Mod}(J \text {, Set }) \downarrow F \rightarrow\left[\mathcal{C}^{\text {op }}, \text { Set }\right] \downarrow F
$$

for a model $F$ takes $P \rightarrow F$ to $L P \rightarrow F$. Since the objects $\mathcal{C}(-, U) \rightarrow F$ of $\operatorname{Mod}\left(J\right.$, Set) $\downarrow F$ strongly generate [ $e^{\text {op }}$, Set] $\downarrow F$, it follows from Theorems (3.10) and (7.25) that the left adjoint to the above displayed inclusion preserves finite products. This means $L$ preserves pullbacks of pairs of arrows with codomains models. If $G$ is a subfunctor of a model $F$ then the unit $G \rightarrow L G$ is a monomorphism, so a pullback into $G$ gives a pullback into $L G$ (a model) and with image under $L$ the same as the image of the original pullback into $G$.

(7.27) Recall (SGA4 [1]) that the left adjoint for the inclusion $\operatorname{Mod}(J$, Set) $\rightarrow$ [ $\bigodot^{\text {op }}$, Set] preserves finite limits when $J$ is a Grothendieck topology on a small category. The question arises as to whether all locally presentable internally-complete categories are Grothendieck topoi. The answer is "no". We give two examples. $^{2}$

(7.28) The category 2 is locally presentable and internally complete yet is not a Grothendieck topos. Let $J$ be the Gabriel theory on 1 for which $\bar{J}(0)$ consists only of the unique function $2 \rightarrow \mathbf{1}(-, 0)$. Then $J$ is pullback stable and $\operatorname{Mod}(J$, Set $) \simeq 2$. So 2 is locally presentable and internally complete (7.25). The monomorphism $0 \rightarrow 1$ in $\mathbf{2}$ is not an equalizer, so $\mathbf{2}$ is not a topos.

(7.29) The full subcategory Mono(Set) of $\left[2^{\text {op }}\right.$, Set] consisting of the monomorphisms is locally presentable and internally complete. There is a pullback stable Gabriel theory $J$ on 2 for which $\bar{J}(0)$ consists only of the identity arrow and $\bar{J}(1)$ consists only of the unique arrow $R \rightarrow 2(-, 1)$ with $R_{1}=2, R_{0}=1$. An object $P$ of [2 $2^{\text {op }}$, Set] is a model for $J$ if and only if $P_{1} \rightarrow P_{0}$ is a monomorphism. So Theorem (7.25) applies with $\mathcal{Q}=\operatorname{Mono}$ (Set). An arrow $\theta: F \rightarrow G$ in $Q$ is a monomorphism if and only if $\theta_{0}$ (and hence $\theta_{1}$ ) is a monomorphism. Yet $\theta$ is a regular monomorphism (equalizer) in $\mathcal{Q}$ if and only if the square

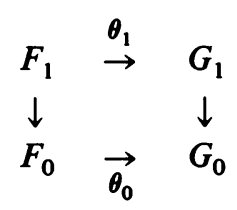

is a pullback. So not every monomorphism is regular; so $Q$ is not a topos. The object $\partial_{2}: 2 \rightarrow 3$ of $\mathbb{Q}$ classifies the regular subobjects, so Mono(Set) is a quasi-topos in the sense of Penon [23].

\footnotetext{
${ }^{2}$ Added in proof: P. T. Johnstone has pointed out how to use (7.25) to prove that a locally presentable category is internally complete if and only if it is a quasi-topos [23]. The models of a pullback stable Gabriel theory in Set are the separated objects for some Lawvere-Tierney topology on some Grothendieck topos.
} 
(7.30) An internal full subcategory of [ $\mathcal{C}^{\text {op }}$, Set] gives rise to a pullback stable Gabriel theory on $\mathcal{C}$ and then to a category of models. While this category of models is of some interest (as in (7.29)), it does not allow us to recapture the internal full subcategory (see (7.18)); while the category of models is an invariant of the internal full subcategory, it is not a complete invariant. Instead of functors $F$ : $e^{\text {op }} \rightarrow$ Set which are orthogonal to the appropriate $\alpha: R \rightarrow \mathcal{C}(-, U)$ as in the model condition (7.9) it is better to consider functors $F$ : ${ }^{\circ o p} \rightarrow$ Cat which are cocomplete with respect to $\alpha$; this means that each arrow $R \rightarrow F$ has a pointwise left kan extension (Street [27]) along $\alpha$. Note that, if $F$ factors through Set, it is cocomplete with respect to $\alpha$ precisely when it is orthogonal to every pullback of $\alpha$ along arrows $\mathcal{C}(-, V) \rightarrow \mathcal{C}(-, U)$. This point will be pursued elsewhere; however, also see (9.11), (9.12), (10.8).

8. Internal full subcategories of locally presentable categories.

(8.1) For a Gabriel theory $J$ on a category $\mathcal{C}$ and for any category $\mathcal{X}$, the assignment $U \mapsto \operatorname{Mod}\left(J_{\mathcal{C}_{\downarrow} U}, \mathcal{X}\right)$ describes a full subfunctor of $\left[\left(\mathcal{C}_{\downarrow} \sim\right)^{\text {op }}, \mathfrak{X}\right]$ : Cop $\rightarrow$ CAT (see (7.19)).

(8.2) Proposition. For a functor P: eop $\rightarrow$ Set, the natural isomorphism (1.2) of categories

$$
\left[\mathcal{G}_{1}^{\mathcal{C}}(P)^{\mathrm{op}}, \mathfrak{X}\right] \cong\left[\mathcal{C}^{\mathrm{op}}, \mathbf{S E T}\right]\left(P,\left[(\mathcal{C} \sim)^{\mathrm{op}}, \mathfrak{X}\right]\right)
$$

restricts to a natural isomorphism of categories

$$
\operatorname{Mod}\left(J_{\mathcal{G}(P)}, \mathscr{X}\right) \cong\left[\mathcal{C o p}^{\mathrm{SETT}}\right]\left(P, \operatorname{Mod}\left(J_{\mathcal{C}_{\downarrow}}, \mathscr{X}\right)\right)
$$

Proof. We must show that $T: \mathcal{G}(P)^{\mathrm{op}} \rightarrow \mathcal{X}$ is a model for $J_{\mathcal{G ( P )}}$ if and only if the corresponding $N: P \rightarrow\left[\left(C_{\downarrow} \sim\right)^{\text {op }}, \mathfrak{X}\right]$ is such that each $N_{U} s$ is a model for $J_{\mathcal{C} \sim}$. But $\left(\left(N_{U} s\right) f=T(V, P f) s\right)$ for $f: V \rightarrow U$ and $s \in P U$. Also $T$ is a model if and only if, for all $\tau: D \rightarrow V$ in $J(V)$ and all $f, s$, the cone with components

$$
T(V,(P f) s) \stackrel{T \tau_{i}}{\rightarrow} T\left(D i, P\left(f \tau_{i}\right) s\right)
$$

is a limit cone.

(8.3) Theorem. Suppose $J$ is a Gabriel theory on a small category $e$ such that the representables $\mathcal{C}(-, U)$ are models for $J$. If $S$ is a model for $J$ in Cat and $\iota:$ $S \rightarrow \operatorname{Mod}\left(J_{\bigcup_{\downarrow} \sim}\right.$, Set) is a pseudo-natural-transformation with fully faithful components then there exists an internal full subcategory $(S, I)$ of $\operatorname{Mod}(J$, Set) which is unique up to isomorphism with the property that $\iota$ is isomorphic to the composite:

$$
\begin{aligned}
& S \stackrel{\sim}{\rightarrow} \operatorname{Mod}(J, \text { Set })(\mathcal{Y} \sim, S) \stackrel{I_{\mathscr{g}} \sim}{\rightarrow} \operatorname{Mod}(J, \text { Set }) \downarrow \mathscr{Y} \sim \\
& \stackrel{\underset{(7.21)}{\longrightarrow}}{\rightarrow} \operatorname{Mod}\left(J_{\mathcal{C} \sim}, \text { Set }\right) .
\end{aligned}
$$


Proof. For a model $F$ of $J$ in Set we have the composite:

$$
\begin{aligned}
& \operatorname{Mod}(J, \operatorname{Set})(F, S)=\left[e^{\text {op }}, \mathbf{S E T}\right](F, S) \\
& \stackrel{\left[{ }^{\text {op }}, \operatorname{SET}\right](i, \iota)}{\rightarrow}\left[e^{\text {op }}, \operatorname{SET}\right]\left(F, \operatorname{Mod}\left(J_{\mathcal{C}_{\downarrow} \sim}, \text { Set }\right)\right) \\
& \underset{(8.2)}{\stackrel{\approx}{\rightarrow}} \operatorname{Mod}\left(J_{\mathcal{G}(F)}, \text { Set }\right) \underset{(7.21)}{\stackrel{\simeq}{\rightarrow}} \operatorname{Mod}(J, \text { Set }) \downarrow F,
\end{aligned}
$$

which is fully faithful and pseudo-natural in $F$. So it is induced by a profunctor $I$ (5.18) and we obtain $(S, I)$ as required. We recapture ı up to isomorphism by substituting $\mathcal{C}(-, U)$ for $F$.

(8.4) One might hope after Theorem (8.3) to obtain a "gross" internal full subcategory for $\operatorname{Mod}\left(J\right.$, Set) as we did for [ [ ${ }^{\text {op }}$, Set] after Theorem (7.5). The situation here is far less satisfactory: it is most unusual for $\operatorname{Mod}\left(J_{\mathcal{C}_{\downarrow} \sim}\right.$, Set) to be a "pseudo-model" for $J$ in CAT (see (8.5)) let alone a model. (The reader will see this for $J$ as in (7.29) for example.)

(8.5) A pseudo-functor $F$ : $e^{\text {op }} \rightarrow \mathcal{K}$ (where $\mathcal{K}$ is a 2-category) is said to be $a$ pseudo-model for $J$ in $\mathcal{K}$ when, for each $\tau: D \Rightarrow U$ in $J(U)$, the pseudo-natural transformation $F \tau: F U \Rightarrow F D$ is a "pseudo-pseudo-limit" for $F D$ in the sense that it induces an equivalence between the category $\mathcal{K}(X, F U)$ and the category of pseudo-natural transformations and modifications from $X$ to $F D$. We say that $F$ is essentially a model for $J$ when it is pseudo-naturally equivalent to a functor eop $\rightarrow \mathscr{K}$ which is a model for $J$ in $|\mathcal{K}|$. If $F$ is essentially a model then it is a pseudo-model but not conversely.

(8.6) Proposition. A pseudo-functor $F$ : $C^{o p} \rightarrow$ CAT is a pseudo-model for $J$ if and only if each $\alpha: R \rightarrow \mathcal{C}(-, U)$ in $\bar{J}(U)$ (7.14) induces an equivalence between the category $F U$ and the category of pseudo-natural-transformations and modifications from $R$ to $F$.

(8.7) A pseudo-functor $F$ : ${ }^{\text {op }} \rightarrow$ CAT is a pseudo-model for a Grothendieck topology (7.22) $J$ on $\mathcal{C}$ if and only if the corresponding left fibration over $\mathcal{C}(5.7)$ is a champ over the site $(\mathcal{C}, J)$ in the sense of Giraud [16, p. 67].

(8.8) Under the conditions of Theorem (8.3), an internal full subcategory of $\operatorname{Mod}(J$, Set) amounts up to pseudo-natural equivalence to a pseudo-functor $T$ : $e^{\text {op }} \rightarrow$ Cat and a pseudo-natural-transformation $\iota: T \rightarrow \operatorname{Mod}\left(J_{\mathcal{C}_{\downarrow} \sim}\right.$, Set) such that $T$ is essentially a model for $J(8.5)$ and the components of $\iota$ are fully faithful.

(8.9) The remainder of this section will be an application of these results to obtain internal full subcategories of the cartesian closed categories $r$-tplcat $=$ cat $^{r}$ (Set) (3.23) of $r$-tple categories (in Set).

(8.10) We shall give details for the case $r=1$. Write $J^{1}$ for the Gabriel theory $J_{\text {cat }}$ on $\Delta_{+}$(3.16) so that (3.17) cat $=\operatorname{Mod}\left(J^{1}\right.$, Set $) \simeq \mid$ Cat $\mid$. The simplicial category $\operatorname{Mod}\left(J_{\Delta_{+}+\sim}^{1}\right.$, Set) is pseudo-naturally equivalent (7.21) to the pseudo-functor

$$
\operatorname{Mod}\left(J^{1}, \text { Set }\right) \downarrow \mathscr{Y} \sim: \Delta_{+}^{\text {op }} \rightarrow \mathbf{C A T},
$$


and this is none other than the pseudo-simplicial category

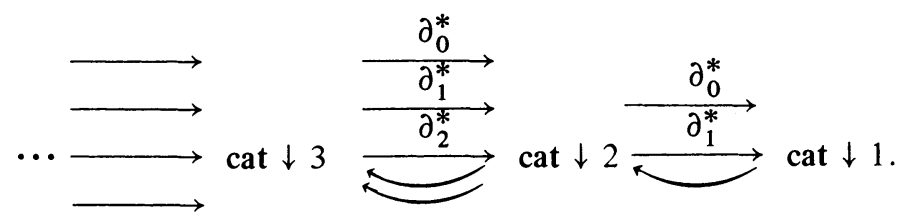

An object of cat $\downarrow \mathbf{n}$ is a functor $f: C \rightarrow \mathbf{n}$ which gives rise to the following data:

(i) for all $i \in \mathbf{n}$, small categories $C^{i}$;

(ii) for all $i<j$, functors $\phi_{j}^{i}: C^{i \text { op }} \times C^{j} \rightarrow$ Set;

(iii) for all $i<j<k$, natural transformations $\phi_{j}^{i} \otimes \phi_{k}^{j} \rightarrow \phi_{k}^{i}$ where

$$
\left(\phi_{j}^{i} \otimes \phi_{k}^{j}\right)(a, c)=\int^{b \in C} \phi_{j}^{i}(a, b) \times \phi_{k}^{j}(b, c)
$$

satisfying the appropriate associativity conditions.

The categories $C^{i}$ are obtained by pullback of $f$ along $i: 1 \rightarrow \mathbf{n}$, the functors $\phi_{j}^{i}$ are given by $\phi_{j}^{i}(a, b)=C(a, b)$, and the natural transformations in (iii) are induced by the composition functions

$$
C(a, b) \times C(b, c) \rightarrow C(a, c) .
$$

Conversely, given (i), (ii), (iii) we can construct a functor $f: C \rightarrow \mathbf{n}$ as follows. The set of objects of $C$ is the disjoint union of the sets of objects of the $C^{i}$. For $x \in C^{i}$, $y \in C^{j}$ the homset $C(x, y)$ is $C^{i}(x, y)$ when $i=j, \phi_{j}^{i}(x, y)$ when $i<j$, and 0 when $j<i$. The compositions of the $C^{i}$ and the natural transformations (iii) provide the composition for $C$. The functor $f: C \rightarrow \mathbf{n}$ is given by $f x=i$ for $x \in C^{i}$. When $\mathbf{n}=2$ we see (4.7), (4.14), (1.9) that an object of cat $\downarrow 2$ is essentially a profunctor between categories in Set.

(8.11) A pseudo-model (8.5) for $J^{1}$ in $\mathscr{K}$ is called a pseudo-category in $\mathscr{K}$; such structures have been considered in the context of "indexed categories" by Wood [32]. In order to obtain a pseudo-category in CAT, for each $n$ we consider the full subcategory psfun(n) of cat $\downarrow \mathbf{n}$ consisting of those functors $f: C \rightarrow \mathbf{n}$ such that the natural transformations $\phi_{j}^{i} \otimes \phi_{k}^{j} \rightarrow \phi_{k}^{i}$ are isomorphisms for all $i<j<k$ in $\mathbf{n}$. Then $\operatorname{psfun}(\sim)$ becomes a sub-pseudo-simplicial category of cat $\downarrow \sim$ which takes the cocones in $J^{1}$ to "pseudo-pseudo-pullbacks"; so it is a pseudo-category in CAT. One may call psfun the gross internal full sub-pseudo-category of cat. It is almost a double category whose objects are small categories, whose vertical arrows are functors, and whose horizontal arrows are profunctors; however, horizontal composition is only associative up to isomorphism. Each functor $h: C^{1} \rightarrow C^{0}$ yields a profunctor from $C^{1}$ to $C^{0}$ determined by $C^{0}(\sim, h-): C^{0 \text { op }} \times C^{1} \rightarrow$ Set, and composition of functors is strictly associative. This suggests a natural full subcategory of the pseudo-category psfun in CAT which we now distinguish.

(8.12) The double category fun which follows is well known (Ehresmann). We shall describe it as a category in CAT: 


$$
\cdots \underset{{ }_{\longrightarrow}^{\longrightarrow}}{\longrightarrow} \text { fun } 2 \stackrel{\frac{d_{0}}{d_{1}}}{\stackrel{d_{2}}{\longrightarrow}} \text { fun } \frac{d_{0}}{\frac{d_{1}}{i_{0}}} \text { fun } \text { fun }_{0}
$$

The category fun is cat. The objects of fun $_{1}$ are arrows $x: C^{1} \rightarrow C^{0}$ in cat. The

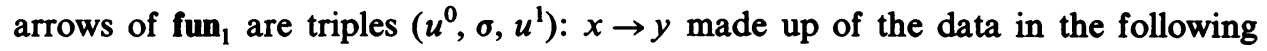
diagram in the 2-category Cat:

$$
\begin{array}{lll}
C^{1} & \stackrel{u^{1}}{\rightarrow} & D^{1} \\
x \downarrow & \stackrel{\sigma}{\Rightarrow} & \downarrow y \\
C^{0} & \overrightarrow{u^{0}} & D^{0}
\end{array}
$$

Composition in fun $_{1}$ is given by

$$
\left(v^{1}, \tau, v^{0}\right)\left(u^{1}, \sigma, u^{0}\right)=\left(v^{1} u^{1}, \tau \cdot \mid \cdot \sigma, v^{0} u^{0}\right)
$$

where $\tau \cdot \mid \cdot \sigma$ is the composite

$$
v^{0} u^{0} x \stackrel{v^{0} \sigma}{\rightarrow} v 9 u^{1} \stackrel{\tau u^{1}}{\rightarrow} z v^{1} u^{1}
$$

The functors $d_{0}, d_{1}:$ fun $\rightarrow$ cat are given on arrows by $d_{0}\left(u^{0}, \sigma, u^{1}\right)=u^{1}, d_{1}\left(u^{0}, \sigma\right.$,

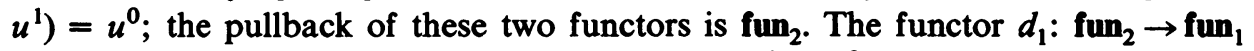
takes $\left(u^{2}, \sigma^{2}, u^{1}, \sigma^{1}, u^{9}\right)$ to $\left(u^{2}, \sigma^{1}+\sigma^{2}, u^{0}\right)$ where $\sigma^{1}+\sigma^{2}$ is the composite

$$
u^{0} x^{1} x^{2} \stackrel{\sigma^{1} x^{2}}{\rightarrow} y^{1} u^{1} x^{2} \stackrel{y^{1} \sigma^{2}}{\rightarrow} y^{1} y^{2} u^{2}
$$

that $d_{1}$ is a functor follows from the middle-four interchange law. Associativity and identity laws are easily checked. The objects if fun $_{n-1}$ (3.22) are strings

$$
C^{n-1} \stackrel{x^{n-1}}{\rightarrow} C^{n-2} \stackrel{x^{n-2}}{\rightarrow} \cdots \stackrel{x^{1}}{\rightarrow} C^{0}
$$

we write $x_{j}^{i}: C^{j} \rightarrow C^{i}$ for the composite $x^{i+1} x^{i+2} \cdots x^{j}$ when $i<j$. The arrows of fun $_{n-1}$ are $(2 n-1)$-tuples

$$
\left(u^{n-1}, \sigma^{n-1}, u^{n-2}, \sigma^{n-2}, \ldots, u^{1}, \sigma^{1}, u^{0}\right) .
$$

(8.15) There is a pseudo-natural transformation $\iota$ : fun $\rightarrow$ cat $\downarrow \sim$ with fully faithful components which we now describe. The functor $\iota_{1}$ is the identity of cat. The functor $\iota_{n}:$ fun $_{n-1} \rightarrow$ cat $\downarrow \mathbf{n}$ takes the object (8.13) to the functor $f: C \rightarrow \mathbf{n}$ determined as in (8.10) by the following data:

(i) the categories $C^{i}$ for $i \in \mathbf{n}$;

(ii) the functors $C^{i}\left(\sim, x_{j}^{i}-\right)$ : $C^{i \text { op }} \times C^{j} \rightarrow$ Set for $i<j$;

(iii) the natural isomorphisms with the canonical components

$$
\int^{b} C^{i}\left(a, x_{j}^{i} b\right) \times C^{j}\left(b, x_{k}^{j} c\right) \simeq C^{i}\left(a, x_{k}^{i} c\right) .
$$

For two strings (8.13) one sees easily that an arrow in cat $\downarrow \mathbf{n}$ between the so-obtained functors into $n$ precisely amounts to an arrow (8.14) of fun fur-1 $_{\text {been }}$ the two strings. 
(8.16) By taking $q:$ obj $\rightarrow$ cat to be the top composite in the following diagram in which the square is a pullback, we obtain a profunctor obj from cat to 1 in $|\mathbf{C A T}|$ (4.7).

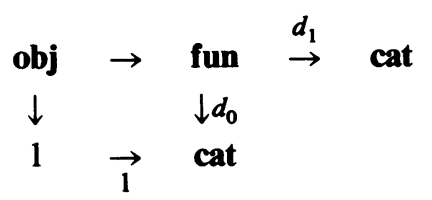

(8.17) Corollary. Suppose $j: S \rightarrow$ fun is a fully faithful functor between categories in $\mid$ CAT|. If $S$ is actually a category in cat then $(S, I)$ is an internal full subcategory of cat where $I$ is the pullback of $q:$ obj $\rightarrow$ cat along $j_{0}: S_{0} \rightarrow$ cat.

(8.18) Corollary (8.17) can be applied to obtain an internal full subcategory of cat from each internal full subcategory of Set. Recall (7.1) that an internal full subcategory of Set amounts to a small full subcategory $\mathcal{U}$ of Set. Write fun( $(U)$ for the full subcategory of fun in $|\mathbf{C A T}|$ obtained by restricting the objects of fun $_{0}=$ cat to those categories whose sets of arrows are in $\mathscr{U}$. Corollary (8.17) yields an internal full subcategory ( $f u n(U), o b j(U)$ ) of cat. This means that, for all small categories $X$, “pulling $q$ : obj(U) $\rightarrow f u n_{0}(U)=$ cat $(ひ)$ back along" provides a fully faithful functor

$$
\operatorname{cat}(X, f u n(\text { Q })) \rightarrow \operatorname{cat} \downarrow X .
$$

This is essentially the "Yoneda-like lemma" of Gray [17, pp. 290-293]. Note that Theorem (6.4) can be applied to give a result for the more general case where $X$ is a category in cat; that is, a small double category (in particular, a 2-category).

(8.19) We shall now briefly deal with the case of general $r$ referred to in (8.9). Write $J^{r}$ for the Gabriel theory on $\Delta_{+}^{r}$ (3.24) for which $r$-tplcat $=\operatorname{Mod}\left(J^{r}\right.$, Set). An $r$-tpl simplicial object $M: \Delta_{+}^{r \text { op }} \rightarrow \mathbb{Q}$ in $\mathbb{Q}$ is an $r$-tpl category in $\mathbb{Q}$ if and only if, for all $\mathbf{n}^{1}, \ldots, \mathbf{n}^{r-1} \in \Delta_{+}$and all $1 \leqslant i \leqslant r-1$, the simplicial object

$$
M\left(\mathbf{n}^{1}, \ldots, \mathbf{n}^{i-1},-, \mathbf{n}^{i+1}, \ldots, \mathbf{n}^{r-1}\right)
$$

is a category in $Q$ (3.22).

(8.20) By (8.3), (8.8), to obtain an internal full subcategory of $r$-tplcat we must produce an $r$-tpl category in cat which has a pseudo-natural transformation into the pseudo-r-tpl-simplicial category

$$
r \text {-tplcat } \downarrow \text { O } \sim: \Delta_{+}^{r \text { op }} \rightarrow \text { CAT }
$$

with fully faithful components. A deductive procedure can be applied as in the case $r=1$ above. We shall just outline the result of the deduction.

(8.21) Write $\mathcal{T}$ for the cartesian closed category $r$-tplcat (3.24). Suppose $\mathcal{T}$ is a $\mathcal{V}$-category in the sense of Eilenberg-Kelly [10]. We shall describe an $r$-tple category $c u(\mathcal{T})$ in $\mid \mathcal{V}$-CAT $\mid$ whose basic ingredients are " $(r+1)$-cubes in $\mathcal{T}$ ". In the first instance $c u(\mathcal{T})$ is a functor

$$
c u(\mathcal{T}): \Delta_{+}^{r \text { op }} \rightarrow|\mathcal{V}-\mathbf{C A T}|,
$$

but since it is to be an r-tple category it will be determined on objects by its value on the full subcategory of $\Delta_{+}^{r \text { op }}$ consisting of those objects $n=\left(n^{1}, \ldots, n^{r}\right) \in \Delta_{+}^{r}$ 
with each $n^{i}=1$ or 2 . For such an $\mathbf{n}$, if $\mathbf{n}^{j}=1$, write $\mathbf{n}(j)$ for the result of replacing $\mathbf{n}^{j}$ by 2 . Define $c u(\mathcal{T})(1, \ldots, 1)$ to be $\mathscr{T}$. Assuming inductively that $c u(\mathcal{T}) \mathbf{n}$ is defined we shall define the $\mathfrak{V}$-category $c u(\mathcal{T}) \mathbf{n}(j)$. The objects are the arrows $x$ : $C^{1} \rightarrow C^{0}$ of $c u(\mathcal{J})$. For two such objects $x: C^{1} \rightarrow C^{0}, y: D^{1} \rightarrow D^{0}$, the r-tple category $(c u(\mathcal{T}) \mathbf{n}(j))(x, y)$ is defined to be the limit in $\mathscr{V}$ of the diagram:

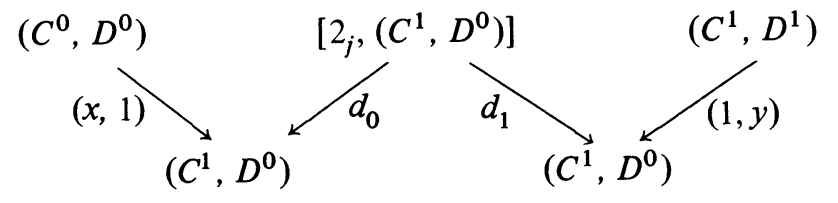

where we have written $(C, D)$ for $(c u(\mathcal{T}) n)(C, D)$ and where $2_{j}$ denotes the $r$-tple category $\Delta_{+}^{r}(-, \mathbf{m}): \Delta_{+}^{r o p} \rightarrow$ Set with $\mathbf{m}^{i}=1$ for $i \neq j$ and $\mathbf{m}^{j}=2$. Composition in $c u(\mathcal{T}) \mathbf{n}(j)$ is induced in an obvious way from that of $c u(\mathcal{T}) \mathbf{n}$. There are obvious domain and codomain arrows $d_{0}, d_{1}: c u(\mathcal{T}) \mathbf{n}(j) \rightarrow c u(\mathcal{T}) n$ in $\mathcal{V}$-CAT taking $x$ : $C^{1} \rightarrow C^{0}$ to $C^{1}, C^{0}$, respectively. These form the underlying graph of an obvious category in $\mid \mathcal{V}$-CAT $\mid$.

(8.22) For the above inductive definition it was necessary to carry through the $\mathfrak{V}$-category structure at each stage to get to the next stage. However we are really interested only in the $r$-tple category $|c u(\mathcal{T})|$ in $|\mathbf{C A T}|$ obtained by composing $c u(\mathcal{T})$ with the underlying functor $|\mathfrak{V}-\mathbf{C A T}| \rightarrow|\mathbf{C A T}|$.

(8.23) Suppose $\mathscr{U}$ is a full subcategory of Set. Then $\operatorname{cat}^{r}(\mathscr{U})(3.23)$ is a full subcategory of $\mathfrak{V}(8.21)$ and hence supports a canonical $\mathfrak{V}$-category structure. Put

$$
\operatorname{fun}^{r}\left(\text { U) }=\mid c u\left(\operatorname{cat}^{r}(\text { U) }) \mid\right.\right.
$$

this is an $r$-tple category in $|\mathbf{C A T}|$, or equivalently, a category in cat $^{r}$ (SET). Note that $f_{u n}{ }^{r}(\mathcal{Q})_{0}=$ fun $^{r}(\mathcal{U}) 1=\operatorname{cat}^{r}(\mathcal{Q})$. When $\mathcal{U}=$ Set we write $r$-tplfun for $f^{r}(\mathcal{Q})$ and define $r$-tplobj by the diagram

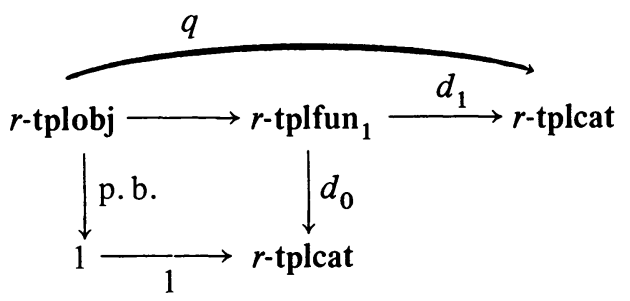

(8.24) When $\mathscr{Q}$ is small, fun $(\mathscr{U})$ is a category in r-tplcat and we obtain a profunctor $\operatorname{obj}^{r}(\mathcal{Q})$ from $\operatorname{cat}^{r}(\mathcal{Q})$ to 1 from the pullback:

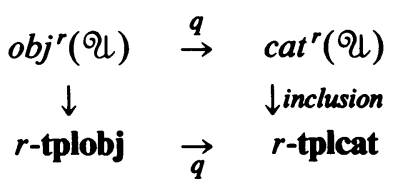

(8.25) THEOREM. For each small full subcategory थ of the category Set of sets, the pair (fun ${ }^{r}(\mathscr{Q}), o b j^{r}(\mathcal{Q})$ ) is an internal full subcategory of the cartesian closed category cat ${ }^{r}$ (Set). 
(8.26) The above construction can be internalized. For any internal full subcategory $(S, I)$ of a finitely complete category $\mathcal{Q}$, we can construct an internal full subcategory $\left(f u n^{r}(S), \operatorname{obj}^{r}(S, I)\right)$ of $\operatorname{cat}^{r}(\mathbb{Q})$.

(8.27) These internal full subcategories of the categories of multiple categories lie at the heart of the comprehension schemes at each level of the hierarchy of r-categories; see Gray [17, pp. 306-310].

9. Locally internal categories. In this section we shall look in more detail at the cosmos structure on [ $\bigodot^{\text {op }}$, Cat] arising via Theorem (6.5) from an internal full subcategory of [ $\bigodot^{o p}$, Set]. We shall treat the case where the internal full subcategory is the realization $(C, I)$ of $\mathcal{C}$ in [£op, Set] (7.7). This example provided motivation for the work of Street [28] and Street-Walters [30]. There has been considerable development of this theory in recent years because of the relationship with topos theory. These connections were made by Lawvere at Perugia, Italy 1972 (although we have been unable to obtain a copy of these notes) and also by Bénabou [4], Lawvere [20], Paré-Schumacher [22], and Celeyrette [5] (also unavailable to the author). We shall not enter the dispute as to whether it is better to work with pseudo-functors, fibrations or indexed categories (with specified canonical isomorphisms). All these points of view can be adequately catered for by a suitable choice of cosmos. The main ideas are present already for the cosmos structure on [ ${ }^{\text {op }}$, Cat $]$ mentioned above, and since $\operatorname{Cat}\left[\bigodot^{\text {op }}\right.$, Set $]=\left[\bigodot^{\text {op }}\right.$, Cat $]$, this example fits the context of the present paper.

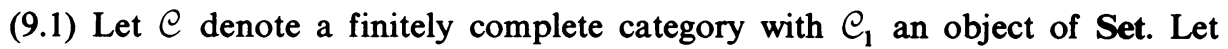
$(C, I)$ denote the realization of $\mathcal{C}$ in [ $\bigodot^{\text {op }}$, Set] as described in (7.7). We shall study the cosmos structure obtained by applying Theorem (6.5) to the internal full subcategory $(C, I)$ of [ $\bigodot^{o p}$, Set]. The underlying 2-category is [ $\bigodot^{o p}$, Cat].

(9.2) In any fibrational cosmos (2.11), a split fibration $E$ from $B$ to $A$ is called admissible (or locally small) when there exist an arrow $h: B \rightarrow \mathscr{P} A$ and an isomorphism $E \cong \in_{A}(A, h)$ over $B \times A$. Admissible split fibrations are necessarily discrete.

(9.3) Proposition. For a discrete fibration $E$ from $B$ to $A$ in the cosmos (9.1), the following are equivalent:

(i) $E$ is admissible;

(ii) for all $U, V \in \mathcal{C}$ and all $a: \mathcal{C}(-, U) \rightarrow A, b: \mathcal{C}(-, V) \rightarrow B$, the fibre $E(a, b)$ of $E$ over $a, b(2.4)$ is representable by an object of $\mathcal{C}$;

(iii) for all $Y, Z \in$ cat(e) and all $a: \mathcal{C}(-, Y) \rightarrow A, b: \mathcal{C}(-, Z) \rightarrow B$, the fibre $E(a, b)$ of $E$ over $a, b$ is representable by a category in $\mathcal{C}$;

(iv) the discrete fibration $\bar{E}$ from $A^{\mathrm{op}} \times B$ to 1 corresponding to $E$ under (4.16) is admissible.

Proof. The functor $\in_{A}(A,-): \mathcal{K}(B, \mathcal{P} A) \rightarrow \operatorname{DSpl}(B, A)$ for this $\operatorname{cosmos}(9.1)$ is equivalent to the composite

$$
\begin{aligned}
& {\left[\bigodot^{\text {op }}, \text { Cat }\right]\left(A^{\text {op }} \times B, C\right) \rightarrow\left[\bigodot^{\text {op }}, \mathbf{C A T}\right]\left(A^{\text {op }} \times B,\left[\left(\bigodot_{\downarrow} \sim\right)^{\text {op }} \text {, Set }\right]\right)} \\
& \stackrel{\sim}{\rightarrow} \operatorname{DSpl}\left(A^{\text {op }} \times B, 1 ;\left[\mathcal{C o p}^{\text {op }} \text { Cat }\right]\right) \text {. }
\end{aligned}
$$


So $E$ is admissible when $\bar{E}$ is isomorphic to an object in the image of the latter composite. This means that, for each $W \in \mathcal{C}, \bar{a} \in A W, \bar{b} \in B W$, the functor $E_{\bar{a} \bar{b}}$ :

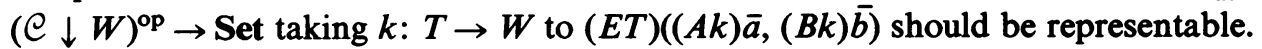

(i) $\Leftrightarrow$ (iv) This is clear since the above composite with $E$ replaced by $\bar{E}$ agrees with that for $E$.

(i) $\Rightarrow$ (ii) Given $a, b$ as in (ii), we obtain, by Yoneda, elements of $A U, B V$ which, using the projections, give elements of $A(U \times V), B(U \times V)$. Applying the above condition with $W=U \times V$ gives a representer for $E(a, b)$.

(ii) $\Rightarrow$ (i) Apply (ii) with $U=V=W$ and $a, b$ corresponding to $\bar{a} \in A W$, $\bar{b} \in B W$. The pullback along the diagonal $W \rightarrow W \times W$ of the representer for $E(a, b)$ gives a representer for $E_{\bar{a} \bar{b}}$.

(iii) $\Rightarrow$ (ii) Take $Y, Z$ discrete.

(ii) $\Rightarrow$ (iii) If $E$ satisfies (ii) so does $2 \pitchfork E$ and hence, in the notation of (iii), $E(a, b)_{1}$ is representable. So $E(a, b)$ is representable by a category in $\mathcal{C}$.

(9.4) Corollary. An object $A$ of the cosmos (9.1) is admissible if and only if, for all objects $U$ of $\mathcal{C}$ and $a, b$ of $A U$, there exist an object $a \pitchfork b$ over $U$ and $a$ natural bijection between arrows

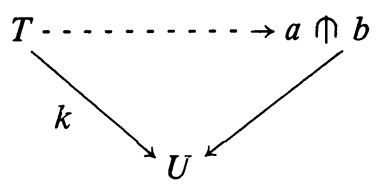

in $\mathcal{\downarrow} U$ and arrows $(A k) a \rightarrow(A k) b$ in $A T$.

(9.5) It follows that $A$ : $e^{o p} \rightarrow$ Cat is admissible precisely when it is a (strict) "locally internal category over $C$ " in the sense of Penon [24], and this is precisely the same as saying the corresponding fibration $\mathcal{G}(A)$ over $\mathcal{C}$ is "localement petite" in the sense of Bénabou [4].

(9.6) CoRollary. For any category $Z$ in $\mathcal{C}$, the object $\mathcal{C}(-, Z)$ of the cosmos (9.1) is admissible.

Proof. Apply (ii) of (9.3) with $E=2 \pitchfork \mathcal{2}(-, Z) \simeq \mathcal{C}(-, 2 \pitchfork Z)$; the representer of $E(a, b)$ is the comma object of the arrows $U \rightarrow Z, V \rightarrow Z$ corresponding to $a, b$.

(9.7) Corollary. If $Z, Z^{\prime}$ are categories in $\mathcal{C}$, if $j: \mathcal{C}(-, Z) \rightarrow B$ is admissible, and if $b: C\left(-, Z^{\prime}\right) \rightarrow B$ is an arrow in the cosmos (9.1), then the object $j \downarrow b$ of [£op, Cat] is representable by a category in $\mathcal{C}$.

Proof. Apply (9.3) to the admissible $E=j \downarrow B$.

(9.8) Corollary. The object $C$ of the cosmos (9.1) is admissible if and only if $\mathcal{C}$ is internally complete (7.24). 


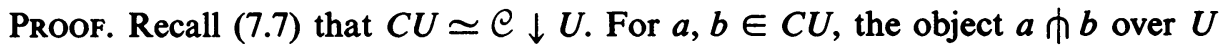
required for admissibility of $C(9.4)$ is precisely a cartesian internal hom in $C \downarrow U$ for the objects corresponding to $a, b$.

(9.9) A functor $F$ : eop $\rightarrow$ Cat is said to have small coproducts when it has the following two properties:

(i) for all $f: V \rightarrow U$, the functor $F f: F U \rightarrow F V$ has a left adjoint $\hat{F} f$;

(ii) for all pullback squares

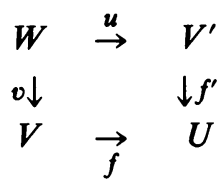

the natural transformation $\hat{F} u \cdot F v \rightarrow F f^{\prime} \cdot \hat{F f}$ (corresponding to the identity $F v \cdot F f$ $\left.=F u \cdot F f^{\prime}\right)$ is an isomorphism.

Condition (i) arises quite often in practice (see Street [26]). Condition (ii) is the familiar condition of Chevalley and Beck which comes up in descent theory. Bénabou [4] says $F$ has "petites sommes".

(9.10) Proposition. If $F$ : $e^{\text {op }} \rightarrow$ Cat has small coproducts then, for all categories $Z$ in $\mathcal{C}$, the category $\mathcal{G}_{1}^{\mathcal{C}}(F) Z(5.13)$ is monadic over $F Z_{0}$ and the underlying functor of the monad is the composite

$$
F Z_{0} \underset{F d_{0}}{\rightarrow} F Z_{1} \underset{\hat{F} d_{1}}{\rightarrow} F Z_{0}
$$

Proof. The unit and multiplication for the monad are obtained from the counits for the adjunctions $\hat{F} i_{0} \dashv F i_{0}, \hat{F} d_{1} \dashv F d_{1}$ by composing on the left with $\hat{F} d_{1}$ and on the right with $F d_{0}$ (besides the simplicial identities and the pseudo-functoriality of $F, \hat{F}$, one uses $\hat{F} d_{2} \cdot F d_{0} \cong F d_{0} \cdot \hat{F} d_{1}$ which comes from (9.9)(ii)). Objects of $\mathcal{G}(F) Z$ are categories in $\mathcal{G}(F)$ which lie over $Z$ and have $d_{0}$ split cartesian; they are determined by their underlying graph:

$$
\left(Z_{1}, e_{1}\right) \underset{\left(d_{1}, \eta\right)}{\stackrel{\left(d_{0,1}, 1\right)}{\rightarrow}}\left(Z_{0}, e_{0}\right)
$$

where $e_{1}=\left(F d_{0}\right) e_{0}, \eta: e_{i} \rightarrow\left(F d_{1}\right) e_{0}$. So they are determined by an object $e_{0}$ and an arrow $\left(\hat{F} d_{1}\right)\left(F d_{0}\right) e_{0} \rightarrow e_{0}$. The condition that this data are an Eilenberg-Moore algebra for the above monad is precisely the condition that the graph should extend to a category in $\mathcal{G}(F)$. The remaining details are left to the reader.

(9.11) Street [27] says that a 2-cell

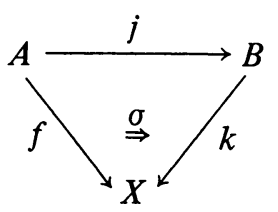


in a finitely complete 2-category exhibits $k$ as a pointwise left (kan) extension of $f$ along $j$ when, for all $b: G \rightarrow B$ and $h: G \rightarrow X$, pasting on the diagram

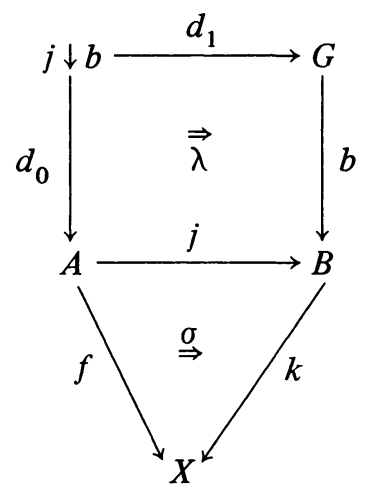

yields a bijection between 2-cells $k b \Rightarrow h$ and 2-cells $f d_{0} \Rightarrow h d_{1}$. We say $X$ is cocomplete with respect to $j$ when every $f: A \rightarrow X$ has a pointwise left extension $k$ along $j$. Compare (7.30).

(9.12) Proposition. An object $F$ of the cosmos (9.1) is cocomplete with respect to all arrows $\mathrm{C}(-, f)$ : $\mathrm{e}(-, V) \rightarrow \mathcal{C}(-, U)$ if and only if $F$ has small coproducts (9.9).

Proof. To say the left extension of $x: \mathcal{C}(-, V) \rightarrow F$ along $\mathcal{C}(-, f)$ exists in the 2-category [ $\left.\mathcal{C}^{\circ}, \mathrm{Cat}\right]$ is to say that $\hat{F} f$ exists at the object of $F V$ corresponding to $x$ under the Yoneda lemma. To test pointwiseness, since $\mathcal{C}(-, U)$ is discrete and the $\left.C_{(-,} V^{\prime}\right)$ are dense in [ ${ }^{\text {op }}$, Set], it suffices to mount pullback squares as in (9.9)(ii) (or rather their image under $\mathcal{Y}$ ) on top of the left extension triangle and ask that the results remain left extensions. The result follows.

(9.13) Let $J$ be the Gabriel theory admitted (7.17) by the internal full subcategory $(C, I)(9.1)$ of [£op, Set]. The elements of $\bar{J}(U)$ are precisely the arrows into $\mathcal{C}(-, U)$ from functors which are representable by objects of $\mathcal{C}(7.17),(9.3)$. So (9.12) shows that $F$ has small coproducts if and only if, for each $U, F$ is cocomplete with respect to all elements of $\bar{J}(U)$. This explains the term "has small coproducts" in analogy with the cosmos arising from Set and a small full subcategory $S$ (for then the elements of $\bar{J}(1)$ are functions $R \rightarrow 1$ where $R$ is a set in $S$, and to say a category $F$ is cocomplete with respect to $R \rightarrow 1$ is to say $F$ has coproducts indexed by $R$ ).

(9.14) An object $X$ of a finitely complete 2-category $\mathcal{K}$ has limits (respectively, colimits) of type $R$, where $R$ is a finitely presented category, when the arrow $X \rightarrow \Re \pitchfork X$ corresponding to the constant functor $\Re \rightarrow \mathscr{K}(X, X)$ at $1_{X}$ has a right (respectively, left) adjoint. For example, taking $R$ to be the free category on the graph $\rightarrow \cdot \leftarrow \cdot$, we see what it means for $A$ to have pullbacks. An object $F$ of [ ${ }^{\circ p}$, Cat] has limits (colimits) of type $R$ if and only if each of the categories $F U$ does and each $F f$ preserves them. 
(9.15) Theorem. Suppose $F \in\left[\bigodot^{\circ p}\right.$, Cat] has small coproducts and coequalizers. Then:

(i) $\mathcal{G}_{1}^{\mathcal{C}}(F) \in\left[\right.$ cat $(\mathcal{C})^{\mathrm{op}}$, Cat $]$ (5.13) has small coproducts and coequalizers;

(ii) $F$ is cocomplete with respect to al admissible arrows $j: \mathcal{C}(-, Z) \rightarrow B$ where $Z$ is a category in $\mathrm{C}^{3}$

Proof. For any functor $u: Z \rightarrow Z^{\prime}$ in $\mathcal{C}$, the vertical functors in the following diagram are monadic (9.10).

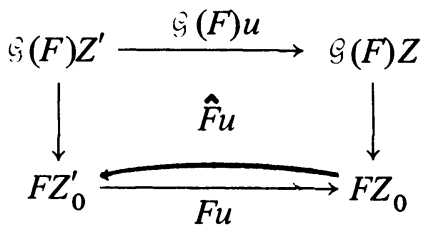

The category $\mathcal{G}(F) Z^{\prime}$ has coequalizers since the underlying functor of the monad on $F Z_{0}^{\prime}$ preserves coequalizers. Dubuc's Adjoint-Triangle Theorem [9] applies to yield a left adjoint $\hat{\mathcal{S}}(F) u$ for $\mathcal{G}(F) u$. Then (i) follows easily.

To obtain a pointwise left extension of $f: \mathcal{C}(-, Z) \rightarrow F$ along $j$ as in (ii) it suffices since the objects $\mathcal{C}\left(-, Z^{\prime}\right)$ are dense in [eop, Cat] to obtain a pointwise left extension of $J \downarrow b \stackrel{d_{0}}{\rightarrow} \mathcal{C}(-, Z) \stackrel{f}{\rightarrow} F$ along $d_{1}: j \downarrow b \rightarrow \mathcal{C}\left(-, Z^{\prime}\right)$ for each $b: \mathcal{C}\left(-, Z^{\prime}\right)$ $\rightarrow B$. By (9.7), $j \downarrow b$ is representable by a category in $\mathcal{C}$. So it suffices to consider the case where $B=C\left(-, Z^{\prime}\right)$. To obtain the pointwise left extension $k$ of $f$ : $\mathcal{C}(-, Z) \rightarrow F$ along $j=\mathcal{C}(-, u): \mathcal{C}(-, Z) \rightarrow \mathcal{C}\left(-, Z^{\prime}\right)$, take the object of $\mathcal{G}(F) Z$ corresponding to $f(5.15)$, apply $\hat{\mathcal{G}}(F) u$ to obtain an object of $\mathcal{G}(F) Z^{\prime}$ which corresponds to an arrow $k: \mathcal{C}\left(-, Z^{\prime}\right) \rightarrow F$ with the desired property.

(9.16) Proposition. Let $M: \mathcal{C} \rightarrow \mathbb{Q}$ be a terminal-object-preserving functor between finitely complete categories and suppose each $\mathbb{\downarrow} \downarrow M U$ is cartesian closed. Let $A: \mathcal{C}^{\mathrm{op}} \rightarrow$ Cat be a functor which is pseudo-naturally equivalent to the pseudo-functor $\mathbb{Q} \downarrow M \sim$ given on arrows by pullback. The object $A$ of the cosmos (9.1) is admissible if and only if $M$ has a right adjoint.

Proof. Suppose $M \dashv N$. Take $a, b \in A U$ and let $h: H \rightarrow M U$ be the cartesian internal hom of the objects of $\mathbb{Q} \downarrow M U$ corresponding to $a, b$. Form the pullback

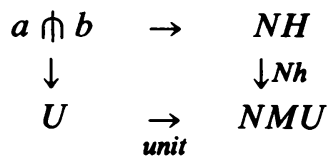

Then $a \pitchfork b$ satisfies the condition of Corollary (9.4).

Conversely, suppose $A$ is admissible. Take $X \in \mathbb{Q}$ and let $N X \rightarrow 1 \simeq M 1$ be $a \pitchfork b$ where $a, b \in A 1$ correspond to $1 \rightarrow M 1, X \rightarrow M 1 \in \mathbb{Q} \downarrow M 1$. This defines a right adjoint $N$ for $M$ on objects.

${ }^{3}$ If $B$ is not representable by a category in $\mathcal{C}$ the left extensions into $F$ may only be pseudo-natural. 


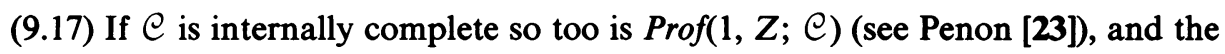
functor $\Delta_{Z}: \mathcal{C} \rightarrow \operatorname{Prof}(1, Z$; $\mathcal{C})$ which takes $U$ to the projection $Z \times U \rightarrow Z$ has a right adjoint called "limit over $Z$ ". The Yoneda lemma gives an isomorphism

$$
\left[\mathcal{e}(-, Z)^{\text {op }}, C\right] U \cong\left[\bigodot^{\text {op }}, \text { Cat }\right]\left(\mathcal{C}\left(-, Z^{\text {op }} \times U\right), C\right),
$$

which, by Theorem (5.15), is isomorphic to $\mathcal{G}_{1}^{\mathcal{C}}(C)\left(Z^{\mathrm{op}} \times U\right)$, and this is equivalent to $\operatorname{Prof}(1, Z \times U ; \mathcal{C}) \cong \operatorname{Prof}(1, Z ; \mathcal{C}) \downarrow \Delta_{Z} U$ by Proposition (9.10). Applying Proposition (9.16) to $\Delta_{Z}$ consequently yields:

(9.18) COROllary. If $Z$ is a category in the internally complete category $\mathcal{C}$ then $\mathcal{P} \hat{\mathcal{Y}} Z \cong\left[\mathcal{C}(-, Z)^{\mathrm{op}}, C\right]$ is admissible in the cosmos (9.1).

(9.19) Recall that an object $A$ of a cosmos was called small by Street-Walters [30, p. 368] when both $A$ and $\mathcal{P} A$ were admissible. By Corollaries (9.6) and (9.18), when $\mathcal{C}$ is internally complete, each category $Z$ in $\mathcal{C}$ yields a small object $\mathcal{C}(-, Z)$ of the cosmos (9.1). It follows for example (by Street-Walters [30, Proposition 13, p. 362] and Street [28, Theorem 27, p. 162]) that, for any arrow $j: \mathcal{C}(-, Z) \rightarrow B$ where $B$ is admissible and $Z$ is a category in $\mathcal{C}$, the arrow $\mathcal{P}_{j}: \mathcal{P} B \rightarrow \mathcal{P}(\mathcal{C}(-, Z))$ has both $a$ right adjoint $\forall j$ and a left adjoint $\exists j$. The objects $\mathcal{C}(-, Z)$ are dense (and hence strongly generating) in [ $\bigodot^{\text {op }}$, Cat], so the theorems of Street [28] which require a strongly generating set of small objects all apply.

(9.20) In the situation of Proposition (9.16) when $M$ has a right adjoint, it has been observed by Bénabou that $A(\simeq \mathbb{Q} \downarrow M \sim)$ has small coproducts if and only if $M$ preserves pullbacks. Since $M$ already preserves terminal objects, $M$ is left exact with a right adjoint. If also $Q$ has coequalizers then so does each $A U$ and each $A f$ preserves them (indeed, pullback along $M f$ has a right adjoint since each $\mathbb{\downarrow} \downarrow M U$ is cartesian closed; Freyd [12]). Thus $A$ is admissible, has small coproducts, and has coequalizers. In particular this applies in the case where $M$ is the inverse image functor of a geometric morphism $Q \rightarrow \mathcal{C}$ between topoi.

(9.21) Write Lex for the 2-category of small finitely complete categories, finitelimit-preserving functors, and natural transformations. Then [£op, Lex] is the 2-category of finitely complete objects $(9.14)$ in [ [ op, Cat].

(9.22) Proposition. The Yoneda structure on the 2-category [ [ ${ }^{\text {op }}$, Cat] arising (2.15) from the fibrational cosmos (9.1) restricts to a Yoneda structure on [£op, Lex].

Proof. Since $C(9.1)$ is in [ $\bigodot^{o p}, \operatorname{Lex}$ ], so is each $\mathscr{P} A$. If $f: A \rightarrow B$ is admissible in the cosmos (9.1) and $B \in\left[\bigodot^{\text {op }}\right.$, Lex] one easily verifies that $\operatorname{hom}_{B}(f, 1)(2.14)$ is an arrow in [ $\bigodot^{\circ p}$, Lex]. So the data (2.14) restrict to [£op, Lex]. Axioms 1 and 2 of Street-Walters [30, pp. 355 and 358] restrict because of the local fullness of the inclusion. Since the inclusion has a left adjoint, a diagram has the absolute left lifting property in [£op, Lex] if and only if it does in [£op, Cat]. It follows that Axiom $3^{*}$ of $[30$, p. 359] holds.

(9.23) If $S: \mathbb{Q} \rightarrow \mathscr{B}$ is a functor such that $\mathcal{Q}$ is small and each of the sets $\mathscr{B}(S A, B)$ is small, we say $S$ is left exact (whether $\mathcal{Q}, \mathscr{B}$ have finite limits or not) when the left adjoint $\exists S:\left[\mathscr{Q}^{\text {op }}\right.$, Set $] \rightarrow\left[\mathscr{B}^{\text {op }}\right.$, Set $]$ to $\left[\mathcal{S}^{\text {op }}\right.$, Set $]$ preserves finite 
limits. If $\mathbb{Q}$ has finite limits this means precisely that $S$ takes finite limits in $\mathbb{Q}$ to finite limits in $\mathscr{B}$.

(9.24) A profunctor $P$ from $B$ to 1 in a finitely complete category $\mathcal{Q}$ is called flat when, for all objects $X$ of $\mathcal{Q}$, the functor (4.18)

$$
P_{X}: \mathbb{Q}(X, B) \rightarrow \mathbb{Q} \downarrow X
$$

is left exact. They form a full subcategory $\operatorname{Flat}(B, \mathbb{Q})$ of $\operatorname{Prof}(B, 1 ; \mathbb{Q})$.

(9.25) Proposition. If $P$ is a flat profunctor from $B$ to 1 in a finitely complete category $\mathbb{Q}$ then, for all categories $A$ in $Q$, the functor (4.17), (2.9)

$$
(\Gamma P)(1, \sim): \operatorname{Cat}(\mathscr{Q})(A, B) \rightarrow \operatorname{DSpl}(A, 1 ; \operatorname{Cat}(\mathbb{Q}))
$$

is left exact.

Proof. Properties of the embedding (4.3) $\hat{\mathcal{Y}}: \operatorname{Cat}(\mathscr{Q}) \rightarrow\left[\mathfrak{Q}^{\text {op }}\right.$, Cat $]$ allow us to reduce the problem to the case where $\mathcal{Q}=\left[\bigodot^{\text {op }}\right.$, Set $]$. Then $P$ amounts to a pseudo-natural transformation $B \rightarrow\left[(\bigodot \downarrow \sim)^{\text {op }}\right.$, Set $]$ with left exact components which can be replaced up to equivalence by a natural transformation $\rho: B^{\prime} \rightarrow H^{\prime}$ with left exact components, so that $(\Gamma P)(1, \sim)$ is isomorphic to the composite

$$
\begin{aligned}
& {\left[\bigodot^{\text {op }}, \text { Cat }\right](A, B) \stackrel{\sim}{\rightarrow}\left[\bigodot^{\text {op }}, \text { Cat }\right]\left(A, B^{\prime}\right) \stackrel{(1, \rho)}{\rightarrow}\left[\bigodot^{\text {op }}, \mathrm{CAT}\right]\left(A, H^{\prime}\right)} \\
& \underset{(1.2)}{\stackrel{\simeq}{\rightarrow}}\left[\mathcal{G}(A-)^{\mathrm{op}}, \text { Set }\right] \stackrel{\simeq}{\rightarrow} \operatorname{DSpl}\left(A, 1 ;\left[\mathcal{C}^{\text {op }}, \text { Cat }\right]\right)
\end{aligned}
$$

which is left exact.

(9.26) Recall that an object $X$ of a 2-category $\mathscr{K}$ is called total relative to a given Yoneda structure on $\mathscr{K}$ when $X$ is admissible and the Yoneda arrow $y_{X}: X \rightarrow \mathscr{P} X$ has a left adjoint. For any small (9.19) object $A$ of $\mathcal{K}$, the object $\mathscr{P} A$ is total (see Street-Walters [30, Corollary 14, p. 363]). If $i: Y \rightarrow X$ is fully faithful, has a left adjoint, and $X$ is total, then $Y$ is total [30, Proposition 27, p. 373].

(9.27) Proposition. For $j: \mathcal{C}(-, Z) \rightarrow B$ as in (9.19), if the components of $j$ are left exact (9.23) then $\exists j: \mathcal{P}(\mathcal{C}(-, Z)) \rightarrow \mathscr{P} B$ is an arrow in [£op, Lex]. Moreover, $\mathscr{P}(\mathcal{C}(-, Z))$ is total in the Yoneda structure (9.22) on [ $\bigodot^{\circ p}$, Lex].

Proof. A cartesian-arrow-preserving functor between right fibrations is left exact if and only if it induces left exact functors on fibres. Thus the cartesian-arrow-preserving functor $J_{U}: \mathcal{G}(\mathcal{C}(-, U) \times \mathcal{C}(-, Z)) \rightarrow \mathcal{G}(\mathcal{C}(-, U)) \times B$ corresponding to $\mathcal{C}(-, U) \times j$ is left exact. The first sentence of the proposition then follows after consideration of the diagram:

$$
\begin{aligned}
& {\left[\mathcal{C}(-, U) \times \mathcal{C}(-, Z)^{\text {op }}, C_{-}\right] \quad \stackrel{(\exists j)_{U}}{\rightarrow} \quad\left[\mathcal{C}(-, U) \times(B-)^{\text {op }}, C_{-}\right]} \\
& {\left[1, \mathscr{Y}_{C_{-}}\right] \downarrow \quad \downarrow\left[1, \mathscr{Y}_{C_{-}}\right]} \\
& {\left[\mathcal{C}(-, U) \times \mathcal{C}(-, Z)^{\text {op }},\left[\left(C_{-}\right)^{\text {op }}, \text { Set }\right]\right] \rightarrow\left[\mathcal{C}(-, U) \times(B-)^{\text {op }},\left[\left(C_{-}\right)^{\text {op }}, \text { Set }\right]\right]} \\
& \simeq \downarrow \quad \downarrow \simeq \\
& {\left[\mathcal{G}(\mathcal{C}(-, U) \times \mathcal{C}(-, Z))^{\text {op }}, \text { Set }\right] \quad \overrightarrow{\exists j}_{U} \quad\left[\mathcal{G}(\mathcal{C}(-, U) \times B)^{\text {op }} \text {, Set }\right]}
\end{aligned}
$$


For the second sentence apply the first sentence with $j$ taken to be the Yoneda arrow of $\mathcal{C}(-, Z)$ in the cosmos (9.1).

(9.28) For objects $A, B$ of [ $\bigodot^{\text {op }}$, Cat], write $\operatorname{Lex}_{\mathrm{e}}(A, B), \operatorname{Geom}_{\mathrm{e}}(A, B)^{\mathrm{op}}$ for the full subcategories of [ $\bigodot^{o p}$, Cat] $(A, B)$ consisting respectively of the arrows $A \rightarrow B$ with left exact components and the arrows $A \rightarrow B$ with left adjoints which have left exact components.

(9.29) Corollary. Suppose $\mathcal{C}$ is internally complete, $Z$ is a category in $\mathcal{C}$, and $B$ is total in the Yoneda structure (9.29) on [£op, Lex]. Then there is an equivalence of categories:

$$
\operatorname{Lex}_{\mathcal{C}}(\mathcal{C}(-, Z), B) \simeq \operatorname{Geom}_{\mathcal{C}}(B, \mathcal{P}(\mathcal{C}(-, Z))),
$$

which takes $j$ to $\operatorname{hom}_{B}(j, 1)=\mathscr{P}_{j} \cdot y_{B}$.

(9.30) Proposition. Suppose $M: \mathcal{Q} \rightarrow \mathbb{Q}$ is a left exact functor between finitely complete categories and suppose $A: e^{\text {op }} \rightarrow$ Cat is a functor pseudo-naturally equivalent to $\mathbb{Q} \downarrow M \sim$. For every category $Z$ in $\mathcal{C}$ there is an equivalence of categories (see (9.24), (9.28)):

$$
\operatorname{Lex}_{\mathfrak{C}}(\mathcal{C}(-, Z), A) \simeq \operatorname{Flat}(M Z, \mathscr{Q}) .
$$

Proof. Since $A \simeq \mathbb{Q} \downarrow M \sim$, we have $\mathcal{G}(A) \simeq \mathbb{Q} \downarrow M$ from which it follows easily (5.13), (4.17) that we have $\mathcal{G}(A) Z \simeq \operatorname{Prof}(M Z, 1 ; \mathcal{Q})$. Theorem (5.15) then gives an equivalence

$$
\left[\bigodot^{\text {op }} \text {, Cat }\right](\mathcal{C}(-, Z), A) \simeq \operatorname{Prof}(M Z, 1 ; \mathbb{Q})
$$

which restricts to that of the proposition.

(9.31) An object $B$ of $\left[\complement^{\circ p}, \mathbf{L e x}\right]$ is said to be bounded when there exist a category $Y$ in $\mathcal{C}$ (internally complete) and a fully faithful arrow $i: B \rightarrow \mathcal{P}(\mathcal{C}(-, Y)$ ) with a left adjoint $l$ in [ $\bigodot^{o p}$, Lex]. By (9.26), (9.27), such a $B$ is total. Put $\mathscr{B}=B 1$ and let $N: \mathcal{C} \rightarrow \mathscr{B}$ be the composite:

$$
\mathcal{C} \simeq C 1 \underset{\text { diag }}{\rightarrow}\left[\mathcal{C}(-, Y)^{\text {op }}, C\right] 1=\mathscr{P}(\mathcal{C}(-, Y)) 1 \underset{l_{1}}{\rightarrow} B 1=\mathscr{B}
$$

Then $N$ is left exact and has a right adjoint; also $\mathscr{B} \downarrow N \sim \simeq B$. If $\mathcal{C}$ is an elementary topos then $\mathscr{B}$ is a bounded topos over $C$ in the sense of Diaconescu [8]; also (9.29), (9.30) combine to yield Diaconescu's result concerning the equivalence of $\operatorname{Flat}(N Z, \mathscr{B})$ and the category of geometric morphisms over $\mathcal{C}$ between the two topoi $\mathscr{B}$ and $\operatorname{Prof}(1, Z ; \mathcal{C})$.

10. Reflective internal full subcategories.

(10.1) Suppose $\mathcal{Q}$ is a finitely complete category. An internal full subcategory $(S, I)$ of $\mathbb{Q}$ is said to be weakly reflective when each of the functors $I_{X}: \mathcal{Q}(X, S) \rightarrow$ $Q \downarrow X$ has left adjoint $L_{X}$. It is reflective when the left adjoints $L_{X}$ are pseudo-natural in $X$.

(10.2) An ordered object of $\mathbb{Q}$ is a category $B$ in $\mathbb{Q}$ for which

$$
\left(\begin{array}{l}
d_{0} \\
d_{1}
\end{array}\right): B_{1} \rightarrow B_{0} \times B_{0}
$$

is a monomorphism. 
(10.3) THEOREM. Suppose $\mathbb{Q}$ is a locally small, finitely complete category which has either small powers or small copowers. If $(S, I)$ is a weakly reflective internal full subcategory of $\mathbb{Q}$ then $S$ is an ordered object of $\mathbb{Q}$ and $q: I \rightarrow S_{0}$ is a monomorphism.

Proof. For each $X$, the category $\mathscr{Q} \downarrow X$ has either small powers or small copowers. Since $\mathbb{Q}(X, S)$ is isomorphic to a reflective subcategory of $\mathscr{Q} \downarrow X$, it does too. Since $\mathcal{Q}(X, S)$ is small, the argument of Freyd [11, Chapter 3, Exercise D, p. 78] shows that it must be an ordered set. So $S$ is an ordered object.

Suppose $u, v: X \rightarrow I$ are such that $q u=q v=w$. Then, by the pullback property of $I_{X}(w)$, we obtain arrows $\bar{u}, \bar{v}: X \rightarrow I_{X}(w)$ over $X$. These reflect to two arrows in $\mathcal{Q}(X, S)$ with source $L_{X}\left(1_{X}\right)$ and target $w$. Since $S$ is ordered, these two arrows, and hence $u, v$, are equal. So $q$ is a monomorphism.

(10.4) Let $\mathcal{Q}$ denote a topos with subobject classifier $\Omega$. A Lawvere-Tierney topology on $Q$ is a monad $j$ on $\Omega$ (see Johnstone [18, pp. 76-78]). Since $\Omega$ is an ordered object, $j$ is idempotent. Let $u: \Omega_{j} \rightarrow \Omega$ be the equalizer of $1_{\Omega}, j$, so that there is an $f: \Omega \rightarrow \Omega_{j}$ with $f u=1, u f=j$. In fact, $\Omega_{j}$ is a kleisli and eilenberg-moore object for $j$; so we have that $u$ is fully faithful and $f \dashv u$. There is a pullback:

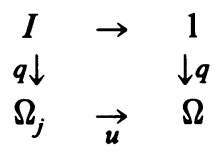

Then $\left(\Omega_{j}, 1\right)$ is an internal full subcategory of $Q(6.8)$.

(10.5) TheOREM. For any Grothendieck topos $\mathcal{Q}$, the assignment $j \mapsto\left(\Omega_{j}, 1\right)$ induces a bijection between the Lawvere-Tierney topologies on $\mathbb{Q}$ and the equivalence classes of reflective internal full subcategories of $\mathbb{Q}$.

Proof. Suppose $(S, I)$ is a reflective internal full subcategory of $\Omega$. By Theorem (10.3), $S$ is ordered and $q: I \rightarrow S_{0}$ is a monomorphism. The characteristic arrow $u$ of this $q$ gives a pullback

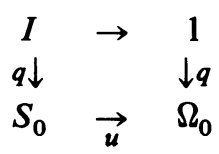

Then $I_{X}: \mathbb{Q}(X, S) \rightarrow \mathbb{Q} \downarrow X$ is isomorphic to the composite

$$
\mathbb{Q}(X, S) \stackrel{\mathbb{Q}(1, u)}{\rightarrow} \mathscr{Q}(X, \Omega) \simeq S u b(X) \rightarrow \mathbb{Q} \downarrow X .
$$

It follows that $u$ is order preserving. Since $I_{X}$ and $S u b(X) \rightarrow Q \downarrow X$ have pseudonatural left adjoints, so too does $\mathbb{Q}(-, u): \mathbb{Q}(-, S) \rightarrow \mathbb{Q}(-, \Omega)$. So $u$ has a left adjoint $f$. This gives a monad $j=u f$ on $\Omega$ with $\left(\Omega_{j}, 1\right)$ equivalent to $(S, I)$.

(10.6) The author does not know whether (10.5) holds for an elementary topos $Q$; the appropriate diagonal argument eludes him.

(10.7) Proposition. Suppose $(S, I)$ is an internal full subcategory of a finitely complete category $\mathbb{Q}$ such that $S$ has a terminal object $*(9.14)$ and each $I_{X}$ : $\mathbb{Q}(X, S) \rightarrow \mathbb{Q} \downarrow X$ preserves terminal objects. Then there is a commutative diagram 


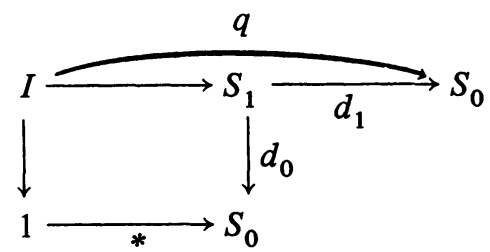

in which the square is a pullback.

Proof. We have the natural bijections:
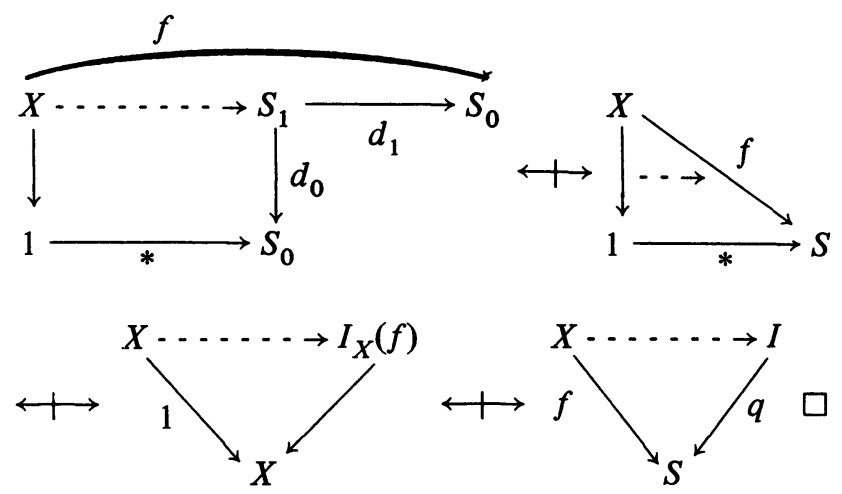

(10.8) THEOREM. An internal full subcategory $(S, I)$ of a finitely complete category $\mathbb{Q}$ is reflective if and only if it satisfies the hypothesis of Proposition (10.7) and $S$ is cocomplete with respect to every arrow of $\mathbb{Q}$ (9.11).

Proof. Suppose $(S, I)$ reflective. Then (10.7) certainly holds. So $I=(* \downarrow S)_{0}$. Take $j: X \rightarrow Y, f: X \rightarrow S$ and notice that $I_{X}(f)=d_{1}:(* \downarrow f)_{0} \rightarrow X$. Let $k$ be the image under $L_{Y}$ of $j d_{1}:(* f)_{0} \rightarrow Y$. For any $h: Y \rightarrow S$, we have natural bijections:
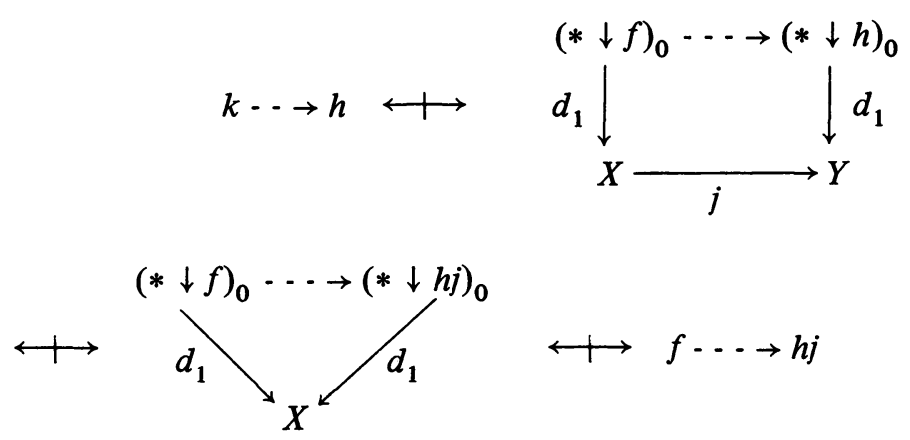

So $k$ is a left extension of $f$ along $j$. That this left extension is pointwise follows from the fact that the following commutes up to isomorphism.

$$
\begin{array}{ccc}
\mathcal{Q}(Y, S) & L_{Y} & \mathbb{\downarrow}(Y \\
\mathcal{Q}(b, 1) \downarrow & & \downarrow b^{*} \\
\mathcal{Q}(G, S) & \leftarrow & \mathbb{\downarrow} \downarrow G
\end{array}
$$


Conversely, one sees that $L_{Y}$ at $j: X \rightarrow Y$ can be taken to be the pointwise left extension of $X \rightarrow 1 \rightarrow S$ along $j$.

(10.9) It follows from the above results that in a nice category $\mathcal{Q}$, if we want an internal full subcategory $(S, I)$ of $\mathbb{Q}$ which mirrors the completeness properties of $\mathcal{Q}$, then the only admissible categories in $\mathcal{Q}$ relative to $(S, I)$ will be ordered objects. We lose all the interesting categories in $\mathcal{Q}$. It is too restrictive to ask that $S$ be cocomplete relative to all arrows of $\mathcal{Q}$. More reasonable completeness conditions on $(S, I)$ (suggested by our $\S 9$ and the canonical example of Set) are the following:

(a) $S$ is finitely complete and finitely cocomplete in Cat $(\mathbb{Q})(9.14)$;

(b) I is flat (9.24) and coflat;

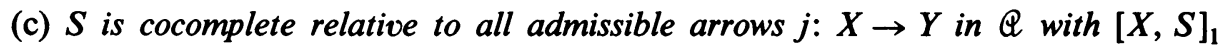
admissible.

Of course one could also require $S$ to be cartesian closed, an elementary topos, etc., and $I$ to "preserve" these essentially algebraic structures.

\section{BIBLIOGRAPHY}

1. M. Artin, A Grothendieck and J. L. Verdier (editors), Théorie des topos et cohomologie etale des schémas, Lecture Notes in Math., vol. 269, Springer-Verlag, Berlin and New York, 1972.

2. A. Bastiani and C. Ehresmann, Categories of sketched structures, Cahiers Topologie Géom. Différentielle 13 (1972), 1-105.

3. J. Bénabou, Théories relatives à un corpus, C. R. Acad. Sci. Paris Sér. A 281 (1975), 831-834.

4. __ Fibrations petites et localement petites, C. R. Acad. Sci. Paris Sér. A 281 (1975), 897-900.

5. J. Celeyrette, Fibrations et extensions de Kan, Thèse de $3^{e}$ cycle, Université Paris-Nord, 1974.

6. B. J. Day, A reflection theorem for closed categories, J. Pure Appl Algebra 2 (1972), 1-11.

7. __ Limit spaces and closed span categories, Lecture Notes in Math., vol. 420, Springer-Verlag, Berlin and New York, 1974, pp. 65-74.

8. R. Diaconescu, Change of base for toposes with generators, J. Pure Appl. Algebra 6 (1975), 191-218.

9. E. Dubuc, Adjoint triangles, Lecture Notes in Math., vol. 61, Springer-Verlag, Berlin and New York, 1968, pp. 69-91.

10. S. Eilenberg and G. M. Kelly, Closed categories, Proc. Conf. Categorical Algebra at La Jolla, Springer-Verlag, Berlin and New York, 1966, pp. 421-562.

11. P. J. Freyd, Abelian categories, Harper \& Row, New York, 1964.

12. __ Aspects of topoi, Bull. Austral. Math. Soc. 7 (1972), 1-76.

13. P. J. Freyd and G. M. Kelly, Categories of continuous functors. I, J. Pure Appl. Algebra 2 (1972), 169-191.

14. P. Gabriel and M. Zisman, Calculus of fractions and homotopy theory, Ergebnisse der Math. und ihrer Grenzgebiete, vol. 35, Springer-Verlag, Berlin and New York, 1967.

15. P. Gabriel and F. Ulmer, Lokal präsentierbare Kategorien, Lecture Notes in Math., vol. 221, Springer-Verlag, Berlin and New York, 1971.

16. J. Giraud, Cohomologie non abélienne, Die Grundlehren der math. Wissenschaften, vol. 179, Springer-Verlag, Berlin and New York, 1971.

17. J. W. Gray, The categorical comprehension scheme, Lecture Notes in Math., vol. 99, SpringerVerlag, Berlin and New York, 1969, pp. 242-312.

18. P. T. Johnstone, Topos theory, London Math. Soc. Monographs, vol. 10, Academic Press, New York, 1977.

19. G. M. Kelly and R. H. Street, Review of the elements of 2-categories, Lecture Notes in Math., vol. 420, Springer-Verlag, Berlin and New York, 1974, pp. 75-103.

20. F. W. Lawvere, Closed categories of variable quantities over a base topos, Notices Amer. Math. Soc. 23 (1976), Abstract \#737-18-8. 
21. S. Mac Lane, Categories for the working mathematician, Graduate Texts in Math., vol. 5, Springer-Verlag, Berlin and New York, 1971.

22. R. Paré and D. Schumacher, Abstract families and the adjoint functor theorems (preprint).

23. J. Penon, Quasitopos, C. R. Acad. Sci. Paris Sér. A 276 (1973), 237-240.

24. __ Catégories localement internes, C. R. Acad. Sci. Paris Sér. A 278 (1974), 1577-1580.

25. R. H. Street, The formal theory of monads, J. Pure Appl. Algebra 2 (1972), 149-168.

26. Two constructions on lax functors, Cahiers Topologie Géom. Différentielle 13 (1972), 217-264.

27. Fibrations and Yoneda's Lemma in a 2-category, Lecture Notes in Math., vol. 420, Springer-Verlag, Berlin and New York, 1974, pp. 104-133.

28. __, Elementary cosmoi. I, Lecture Notes in Math., vol. 420, Springer-Verlag, Berlin and New York, 1974, pp. 134-180.

29. __ Limits indexed by category-valued 2-functors, J. Pure Appl. Algebra 8 (1976), 149-181.

30. R. H. Street and R. F. C. Walters, Yoneda structures on 2-categories, J. Algebra 50 (1978), 350-379.

31. __, The comprehensive factorization of a functor, Bull. Amer. Math. Soc. 79 (1973), 936-941.

32. R. Wood, Indicial methods for relative categories, Ph.D. dissertation, Dalhousie University, Canada, 1976.

School of Mathematics and Physics, Macquarie University, North Ryde, N.S.W. 2113, Australia 